University of Tennessee Health Science Center

UTHSC Digital Commons

$12-2013$

\title{
A Novel Mechanism of Enhanced Susceptibility to Bacterial Pneumonia in Influenza-infected Hosts
}

Hazem Elsayed Ghoneim

University of Tennessee Health Science Center

Follow this and additional works at: https://dc.uthsc.edu/dissertations

Part of the Bacterial Infections and Mycoses Commons, Medical Biochemistry Commons, Medical Immunology Commons, Medical Microbiology Commons, and the Virus Diseases Commons

\section{Recommended Citation}

Ghoneim, Hazem Elsayed, "A Novel Mechanism of Enhanced Susceptibility to Bacterial Pneumonia in Influenza-infected Hosts" (2013). Theses and Dissertations (ETD). Paper 89. http://dx.doi.org/10.21007/ etd.cghs.2013.0108.

This Dissertation is brought to you for free and open access by the College of Graduate Health Sciences at UTHSC Digital Commons. It has been accepted for inclusion in Theses and Dissertations (ETD) by an authorized administrator of UTHSC Digital Commons. For more information, please contact jwelch30@uthsc.edu. 


\title{
A Novel Mechanism of Enhanced Susceptibility to Bacterial Pneumonia in Influenza-infected Hosts
}

\begin{abstract}
Viruses such as influenza suppress host immune function by a variety of methods. This may result in a significant morbidity through several pathways, including facilitation of secondary bacterial pneumonia from pathogens such as Streptococcus pneumoniae. Lungresident alveolar macrophages (AMs) act as the first line of innate cellular immunity against respiratory bacterial pathogens, including pneumococcus. Therefore, they represent an attractive target for study Before investigating the impact of influenza infection on resident AMs, we first characterized different subsets of lung-resident macrophages in naïve mice using a novel in vivo labeling approach in conjunction with multicolor flow cytometric analysis and confocal microscopic examination. A stable fluorescent dye, PKH26-PCL, was administered intranasally to selectively label the lung-resident macrophages in a well-established murine model prior to influenza infection. We determined the turnover kinetics of the lung-resident macrophage subsets during the course of influenza infection. More than $90 \%$ of resident AMs were lost in the first week after influenza, while the remaining cells had a necrotic phenotype. To establish the impact of this innate immune defect, influenzainfected mice were challenged with a small dose of Streptococcus pneumoniae. Early AM-mediated bacterial clearance was significantly impaired during the AM depletion phase in influenza-infected mice about $50 \%$ of the initial bacterial inoculum could be harvested from the alveolar airspaces 3 hours later. In mock-infected mice, by contrast, more than $95 \%$ of inocula up-to-50-fold higher was efficiently cleared.

Co-infection during the AM depletion phase caused significant body weight loss and mortality. Two weeks after influenza, the AM population was fully replenished with successful re-establishment of the early innate host protection. Local GM-CSF treatment induced partial expansion of resident AMs during influenza infection. Thus, it led to partial restoring of the impaired early bacterial clearance with efficient protection against secondary pneumococcal pneumonia. We conclude that a novel immunosuppression mechanism occurs during influenza infection through the resident AM depletion. Among other potential effects, this establishes a niche for secondary pneumococcal infection by altering early cellular innate immunity in the lungs resulting in pneumococcal outgrowth and lethal pneumonia. This novel mechanism will inform development of novel therapeutic approaches to restore lung innate immunity against bacterial super-infections. Secondary bacterial pneumonia (SBP) is a leading cause of the increased hospitalizations and mortality during influenza epidemics and pandemics despite routine use of standard antibiotics. Antibiotic-induced immunopathology associated with bacterial cell wall lysis has been suggested to contribute to these poor outcomes. Using Streptococcus pneumoniae in a wellestablished murine model of SBP following influenza, we stratified disease severity based on the pneumococcal load in the lungs via in vivo bioluminescence imaging. Ampicillin treatment cured mice with mild pneumonia but was ineffective against severely pneumonic mice, despite effective bacterial killing. This treatment failure makes it crucial to explore immunmodulation approaches that can prevent the aggravated lung immunopathology during antibiotic treatment of severe SBP. Therefore, we tested the efficacy of the standard anti-inflammatory drug dexamethasone as an adjunctive corticosteroid therapy. Adjunctive dexamethasone treatment significantly improved ampicillin-induced immunopathology and survival outcomes in mice with severe SBP. However, early dexamethasone therapy during primary influenza infection impaired the adaptive immunity in the lungs as manifest by increased viral titers, with an associated loss of its protective functions in SBP. The clinical use of corticosteroids as an adjunctive therapy for treating pneumonia is still under debate. However, our findings support adjunctive clinical use of corticosteroids in severe cases of community-acquired pneumonia. Nonetheless, dexamethasone treatment has drawbacks implied by delayed body weight recovery in dexamethasone-treated mice, which may explain the published controversy on the corticosteroid efficacy in terms of disease morbidity. This relative success of our animal model of SBP to simulate the clinical therapeutic settings in humans will help explore novel immunomodulation approaches to improve the poor outcomes of antibiotic treatment
\end{abstract}


of severe community-acquired pneumonia.

Document Type

Dissertation

Degree Name

Doctor of Philosophy (PhD)

Program

Biomedical Sciences

Research Advisor

Jonathan A. McCullers, M.D.

\section{Keywords}

Influenza, Alveolar Macrophages, Streptococcus pneumoniae, Secondary Bacterial Pneumonia, Immunomodulation, Corticosteroids.

\section{Subject Categories}

Bacterial Infections and Mycoses | Diseases | Medical Biochemistry | Medical Immunology | Medical Microbiology | Medicine and Health Sciences | Virus Diseases

\section{Comments}

Six month embargo expired June 2014 
A Novel Mechanism of Enhanced Susceptibility to Bacterial Pneumonia in Influenza-infected Hosts

\author{
A Dissertation \\ Presented for \\ The Graduate Studies Council \\ The University of Tennessee \\ Health Science Center \\ In Partial Fulfillment \\ Of the Requirements for the Degree \\ Doctor of Philosophy \\ From The University of Tennessee
}

By

Hazem Elsayed Ghoneim

December 2013 
Portions of Chapters 2 and 3 (C) 2013 by

The American Association of Immunologists, Inc.

All other material (C) 2013 by Hazem Elsayed Ghoneim.

All rights reserved. 


\section{DEDICATION}

To my wife for her love, endless support, and encouragement.

To every child in St. Jude fighting cancer, whose courage and strength inspire me to make the world a better place. 


\section{ACKNOWLEDGEMENTS}

I would like to acknowledge all those who have helped and supported me throughout my graduate studies. I would especially like to thank my advisor, Dr. Jonathan McCullers, for his guidance and mentorship throughout my PhD studies. Jon, you have been an exceptional mentor. Your mentorship guided me through my work with essential discussions, directions and re-directions. I still remember when I asked you in the beginning about what you should expect from me to graduate; your answer was when I developed the critical thinking. Indeed, you have encouraged me to think independently and seek answers to the enduring critical questions in the field by analyzing the literature, developing hypotheses, and testing them using a diverse array of novel approaches.

I would like to thank all my committee members, Dr. Richard Webby, Dr. Paul Thomas, Dr. Christopher Waters, and Dr. Boyce Keith English, who have been very helpful providing me with continuous encouragement and critical guidance throughout my graduate studies. Dr. Paul, thank you for your continuous feedback and experimental suggestions over the years, and for allowing me to use your lab facilities any time for my Immunology work. Dr. Webby, thank you for your experienced guidance and helpful advices since you granted me the opportunity to do a rotation in your lab in 2010 . This facilitated me to have collaborations with various people in your lab, all have been educational. Dr. Waters and Dr. English, many thanks to you for your helpful suggestions, professional guidance, kind encouragement, and all your time over the years!

Additionally, I would like to thank everyone in the McCullers' lab, especially Amy Iverson for her kind technical assistance along the way, in addition to Dr. Amali Samarasinghe and Dr. Irina Alymova for their insightful discussions, continuous encouragement, and didactic career advice.

I would also like to express gratitude to my friends who helped establish my life in Memphis, especially Dr. Ali Ellebedy who helped me a lot since picking me up from the airport, when I first came to Memphis in 2009, until he left for his postdoc career at Emory University in 2011. Ali, you have been a wonderful friend who kept advising me on my career directions. Also, thank you for your technical teaching during my first steps into the Immunology world, while I was doing my rotation in Dr. Webby's lab.

I would like to acknowledge the National Institute of Allergy and Infectious Diseases (NIAID) and the American Lebanese Syrian Associated Charities (ALSAC) for funding my studies through Dr. McCullers. Also, I would like to acknowledge the Flow Cytometry and the Cell and Tissue Imaging Facilities at St. Jude Children's Research Hospital for providing me with the technologies and training I needed.

Finally, I would like to thank my loving wife, Dalia Marzouk, for all her encouragement and support throughout my work and life. Dalia, your love and great help are the cornerstone of our achievements. Additionally, I would like to thank my family 
back home for all their endless love, emotional support, and tremendous encouragement for me to be the best and achieve my goals. 


\begin{abstract}
Viruses such as influenza suppress host immune function by a variety of methods. This may result in a significant morbidity through several pathways, including facilitation of secondary bacterial pneumonia from pathogens such as Streptococcus pneumoniae. Lung-resident alveolar macrophages (AMs) act as the first line of innate cellular immunity against respiratory bacterial pathogens, including pneumococcus. Therefore, they represent an attractive target for study.

Before investigating the impact of influenza infection on resident AMs, we first characterized different subsets of lung-resident macrophages in naïve mice using a novel in vivo labeling approach in conjunction with multicolor flow cytometric analysis and confocal microscopic examination. A stable fluorescent dye, PKH26-PCL, was administered intranasally to selectively label the lung-resident macrophages in a wellestablished murine model prior to influenza infection. We determined the turnover kinetics of the lung-resident macrophage subsets during the course of influenza infection. More than $90 \%$ of resident AMs were lost in the first week after influenza, while the remaining cells had a necrotic phenotype.
\end{abstract}

To establish the impact of this innate immune defect, influenza-infected mice were challenged with a small dose of Streptococcus pneumoniae. Early AM-mediated bacterial clearance was significantly impaired during the AM depletion phase in influenza-infected mice - about $50 \%$ of the initial bacterial inoculum could be harvested from the alveolar airspaces 3 hours later. In mock-infected mice, by contrast, more than $95 \%$ of inocula up-to-50-fold higher was efficiently cleared. Co-infection during the AM depletion phase caused significant body weight loss and mortality. Two weeks after influenza, the AM population was fully replenished with successful re-establishment of the early innate host protection. Local GM-CSF treatment induced partial expansion of resident AMs during influenza infection. Thus, it led to partial restoring of the impaired early bacterial clearance with efficient protection against secondary pneumococcal pneumonia.

We conclude that a novel immunosuppression mechanism occurs during influenza infection through the resident AM depletion. Among other potential effects, this establishes a niche for secondary pneumococcal infection by altering early cellular innate immunity in the lungs resulting in pneumococcal outgrowth and lethal pneumonia. This novel mechanism will inform development of novel therapeutic approaches to restore lung innate immunity against bacterial super-infections.

Secondary bacterial pneumonia (SBP) is a leading cause of the increased hospitalizations and mortality during influenza epidemics and pandemics despite routine use of standard antibiotics. Antibiotic-induced immunopathology associated with bacterial cell wall lysis has been suggested to contribute to these poor outcomes. Using Streptococcus pneumoniae in a well-established murine model of SBP following influenza, we stratified disease severity based on the pneumococcal load in the lungs via 
in vivo bioluminescence imaging. Ampicillin treatment cured mice with mild pneumonia but was ineffective against severely pneumonic mice, despite effective bacterial killing. This treatment failure makes it crucial to explore immunmodulation approaches that can prevent the aggravated lung immunopathology during antibiotic treatment of severe SBP.

Therefore, we tested the efficacy of the standard anti-inflammatory drug dexamethasone as an adjunctive corticosteroid therapy. Adjunctive dexamethasone treatment significantly improved ampicillin-induced immunopathology and survival outcomes in mice with severe SBP. However, early dexamethasone therapy during primary influenza infection impaired the adaptive immunity in the lungs as manifest by increased viral titers, with an associated loss of its protective functions in SBP. The clinical use of corticosteroids as an adjunctive therapy for treating pneumonia is still under debate. However, our findings support adjunctive clinical use of corticosteroids in severe cases of community-acquired pneumonia. Nonetheless, dexamethasone treatment has drawbacks implied by delayed body weight recovery in dexamethasone-treated mice, which may explain the published controversy on the corticosteroid efficacy in terms of disease morbidity. This relative success of our animal model of SBP to simulate the clinical therapeutic settings in humans will help explore novel immunomodulation approaches to improve the poor outcomes of antibiotic treatment of severe communityacquired pneumonia. 


\section{TABLE OF CONTENTS}

CHAPTER 1. INTRODUCTION .............................................................................................1

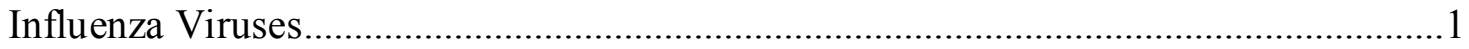

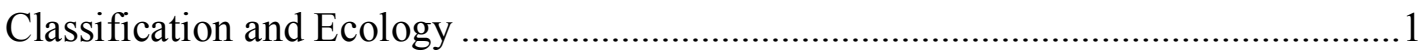

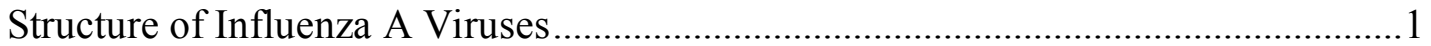

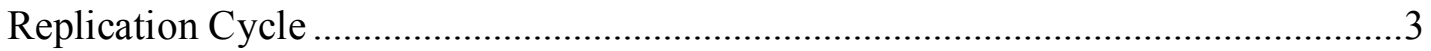

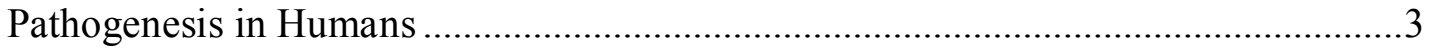

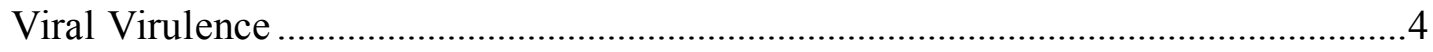

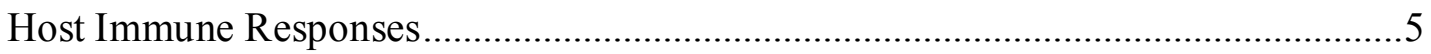

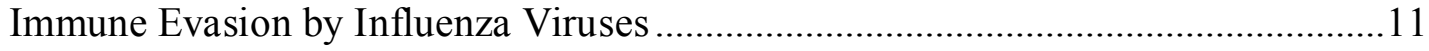

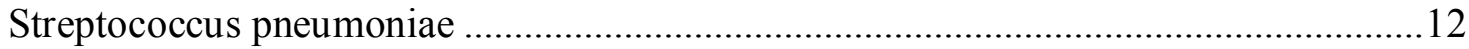

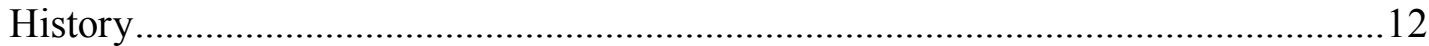

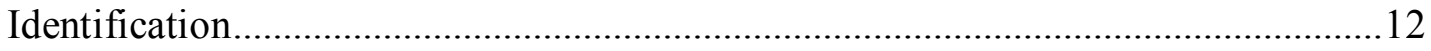

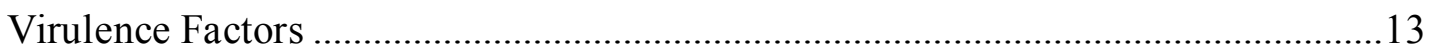

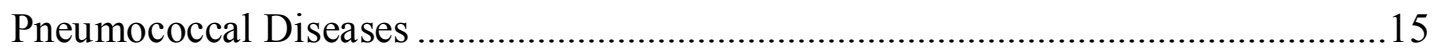

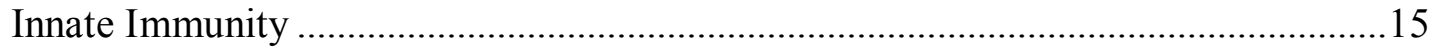

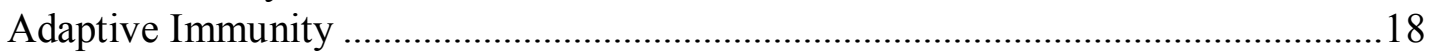

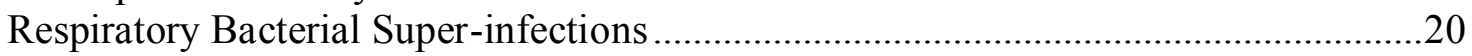

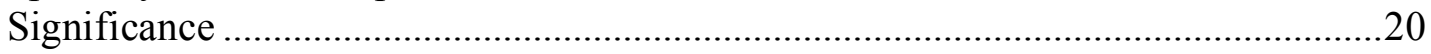

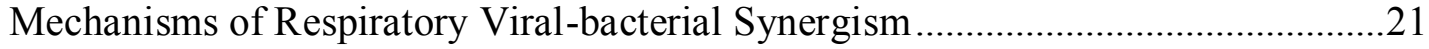

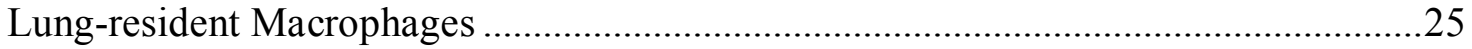

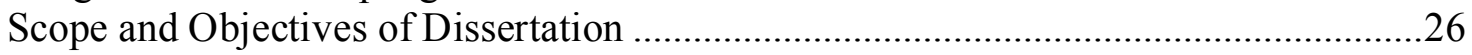

Specific Aim 1: To Determine the Impact of Influenza Infection on the Lung-

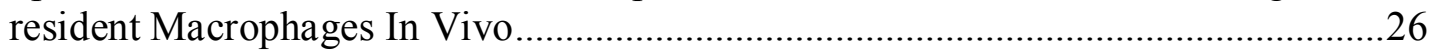

Specific Aim 2: To Determine if Depletion of Resident Alveolar Macrophages during Influenza Infection Contributes to Enhanced Susceptibility to Secondary

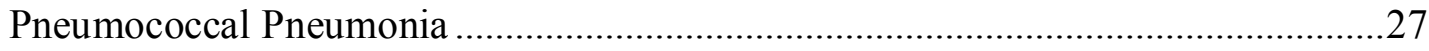

Specific Aim 3: To Test if Expanding the Lung-resident Macrophage Pool Can Protect Influenza-infected Mice against Secondary Pneumococcal Pneumonia .......27 Specific Aim 4: To Test if Adjunctive Immunomodulator Therapy Can Improve Outcomes during Bactericidal Antibiotic Treatment of Secondary Pneumococcal Pneumonia

\section{CHAPTER 2. IMPACT OF INFLUENZA INFECTION ON THE LUNG-} RESIDENT MACROPHAGES IN VIVO ............................................................28

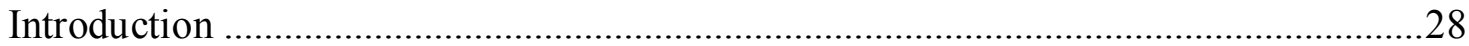

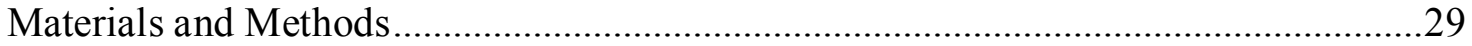

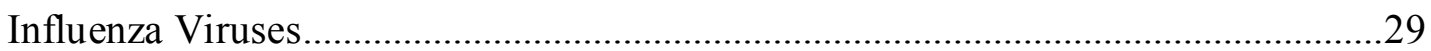

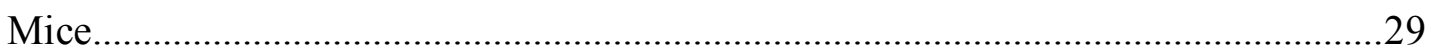

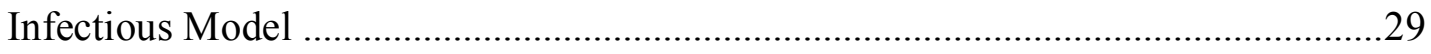

In Vivo Labeling of Lung-resident Macrophages ....................................................... 30

Flow Cytometric Analysis of Immune Cells in Bronchoalveolar Lavage Fluid, Post-lavage Lungs, and Mediastinal Lymph Nodes................................................... 30

Confocal Laser Scanning Microscopy of PKH26-labeled Lungs ............................... 31 


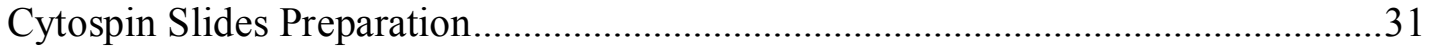

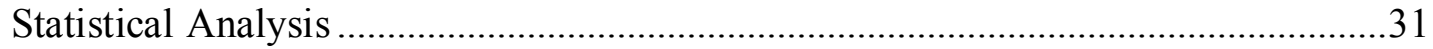

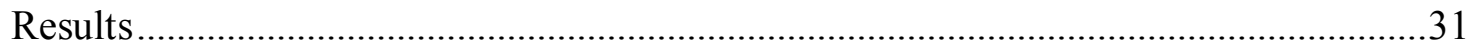

In Vivo Labeling of Lung-resident Macrophages Can Distinguish Alveolar

Macrophages from Interstitial Macrophages .................................................................31

Influenza Virus Infection Depletes Alveolar Macrophages ..........................................32

Tracking of Lung-resident Macrophage Dynamic Changes during Influenza

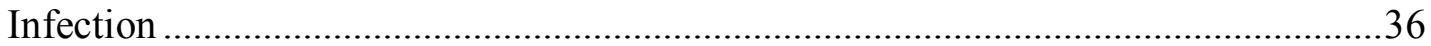

Validity and Stability of the In Vivo Labeling Approach to Distinguish Different

Subsets of Pulmonary Macrophages during the Tracking Period ..................................36

Ontogeny of the Replenished Pool of Alveolar Macrophages after Influenza

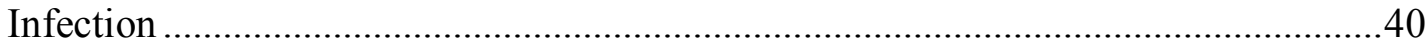

Influenza Infection Induces Cell Death of Alveolar Macrophages...............................40

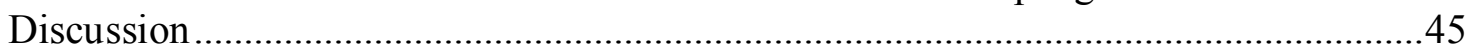

\section{CHAPTER 3. DEPLETION OF RESIDENT ALVEOLAR MACROPHAGES DURING INFLUENZA INFECTION CONTRIBUTES TO ENHANCED SUSCEPTIBILITY TO BACTERIAL PNEUMONIA .......................................................48}

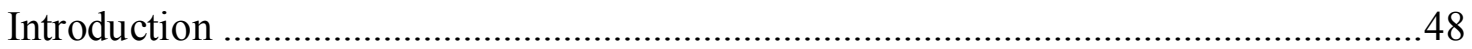

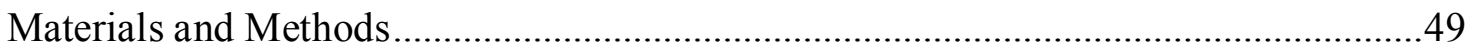

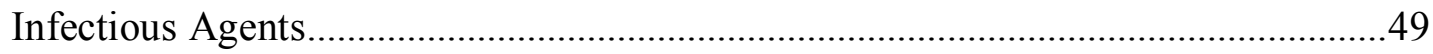

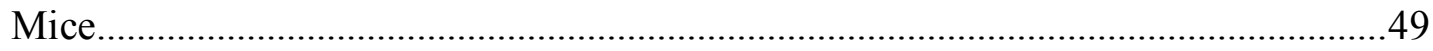

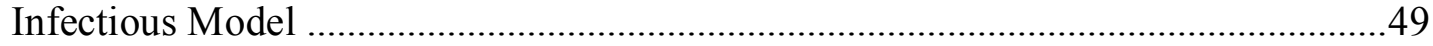

Imaging of Live Mice ...........................................................................................50

Flow Cytometric Analysis of Immune Cells in BALF and Post-lavage Lungs ..........50

Determination of Early Pneumococcal Clearance ………………………………….....50

Local GM-CSF Treatment Regimen ..................................................................50

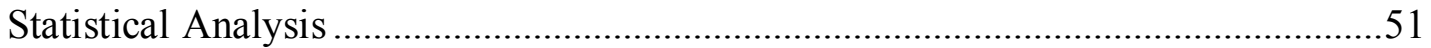

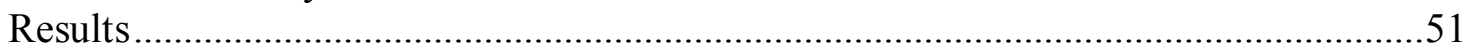

Influenza Infection Enhances Susceptibility to Secondary Pneumococcal

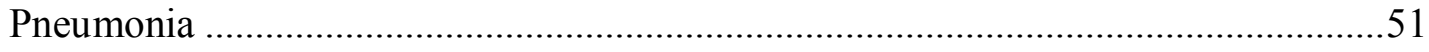

AM Depletion during Influenza Infection Impairs Early Pneumococcal

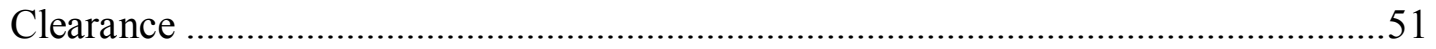

Influenza-infected Mice Demonstrate Increased Susceptibility to Secondary

Pneumococcal Pneumonia during the AM Depletion Phase .........................................53

Local GM-CSF Treatment Expands the Lung-resident Macrophage Pool in

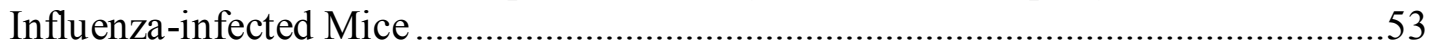

Local GM-CSF Treatment Decreases the Susceptibility to Secondary

Pneumococcal Pneumonia Following Influenza ..........................................................56

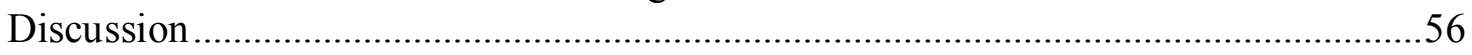

\section{CHAPTER 4. ADJUNCTIVE IMMUNOMODULATOR THERAPY}

IMPROVES MORTALITY DURING ANTIBIOTIC TREATMENT OF

SECONDARY PNEUMOCOCCAL PNEUMONIA ........................................................59

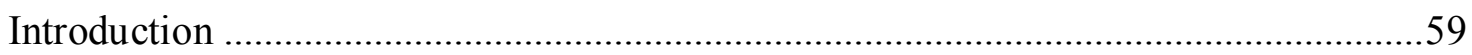

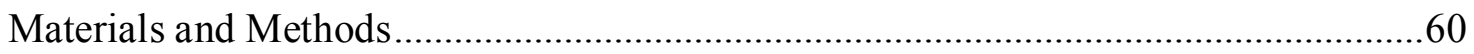




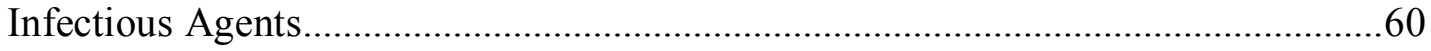

Mice

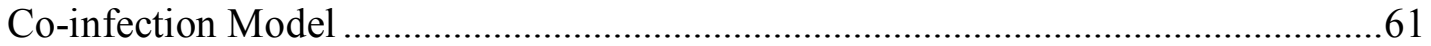

Imaging and Ampicillin Treatment of Live Co-infected Mice ...................................61

Flow Cytometric Analysis of Immune Cells in BALF and Post-lavage Lungs .........61

Dexamethasone Treatment Regimen .......................................................................62

2,3-Diacetyloxybenzoic Acid Treatment Regimen .....................................................62

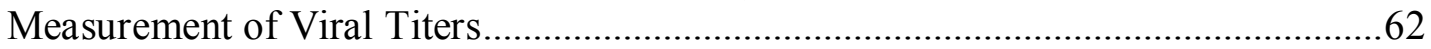

Measurement of Total Protein and Albumin Levels in BALF Supernatant ................63

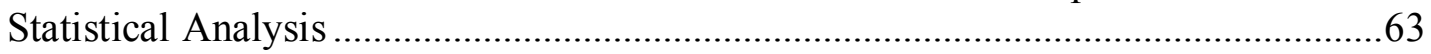

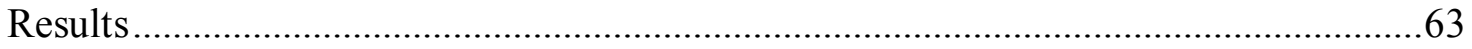

Ampicillin Treatment during Secondary Pneumococcal Pneumonia Fails to

Rescue Mice Despite Viral and Bacterial Clearance

Differential Outcomes Depend on the Severity of Secondary Pneumococcal

Pneumonia at the Onset of Antibiotic Treatment ..........................................................63

Adjunctive Dexamethasone Treatment Rescues Ampicillin-treated Mice with

Severe Secondary Pneumococcal Pneumonia ...............................................................65

Early Dexamethasone Treatment Leads to Loss of Its Protective Activity .................65

Early Dexamethasone Treatment Suppresses Adaptive Immunity ...............................70

Adjunctive DABA Treatment Improves Survival of Ampicillin-treated Mice with Secondary Pneumococcal Pneumonia .................................................................70

Early DABA Treatment Does Not Worsen the Morbidity of Influenza-infected

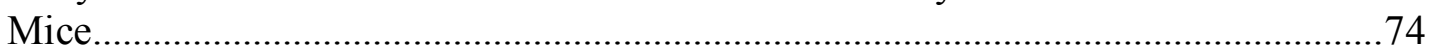

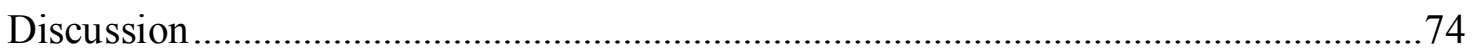

\section{CHAPTER 5. GENERAL DISCUSSION, INSIGHTS, AND FUTURE}

DIRECTIONS .......................................................................................................................78

Impact of Influenza Infections on Resident Alveolar Macrophages In Vivo..................79

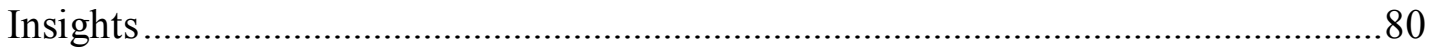

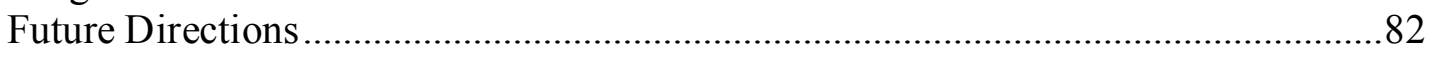

Treatment Strategies of Secondary Bacterial Pneumonia Following Influenza..............83

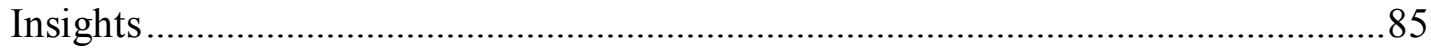

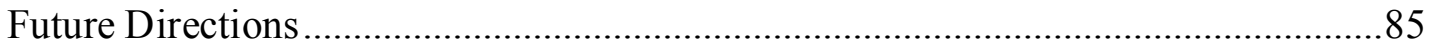

LIST OF REFERENCES ..............................................................................................................87

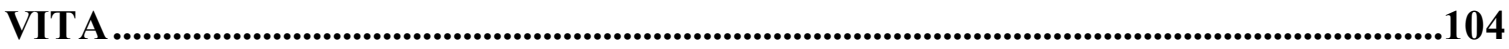




\section{LIST OF FIGURES}

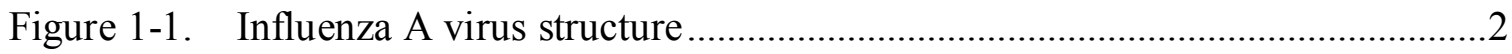

Figure 1-2. Molecular basis of immune responses at site of influenza infection ..............6

Figure 1-3. Kinetics of adaptive immune responses during primary and secondary influenza infections.........................................................................................

Figure 1-4. Adaptive immune response against influenza virus infection .......................10

Figure 1-5. Streptococcus pneumoniae structure and virulence factors ...........................14

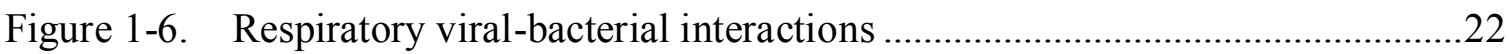

Figure 2-1. Differentiation between lung-resident macrophages subsets.........................33

Figure 2-2. Fluorescence microscopic examination of PKH26-labeled naive lungs confirms selective in vivo labeling of lung-resident macrophages

Figure 2-3. Lung-resident alveolar macrophages are depleted during influenza virus infections

Figure 2-4. Tracking of lung-resident macrophage dynamic changes during influenza infection

Figure 2-5. Dynamic changes of the mean fluorescence intensity of PKH26-PCL of the lung-resident macrophages during influenza infection .............................38

Figure 2-6. Differential mean fluorescence intensity of PKH26-PCL dye of different pulmonary macrophage subsets during influenza infection.

Figure 2-7. Differential surface expression of Ly6C marker on different pulmonary macrophage subsets .

Figure 2-8. Fluorescence intensity of PKH26-PCL dye is stable in lung-resident macrophages for 2 weeks after mock infection

Figure 2-9. Ontogeny of the replenished alveolar macrophage pool during influenza infection

Figure 2-10. Influenza infection induces significant alveolar macrophage cell death......44

Figure 3-1. Murine influenza-pneumococcal co-infection model.....................................52

Figure 3-2. Successful co-infection synergism during the AM depletion phase in influenza-infected mice 
Figure 3-3. Local GM-CSF treatment expands the lung-resident macrophage pool in influenza-infected mice

Figure 3-4. Local GM-CSF treatment decreases the susceptibility to secondary pneumococcal pneumonia following influenza

Figure 4-1. Ampicillin treatment of secondary pneumococcal pneumonia......................64

Figure 4-2. Differential mortality outcomes depend on the severity of secondary pneumococcal pneumonia at the onset of antibiotic treatment

Figure 4-3. Differential lung immunopathology depends on the severity of secondary pneumococcal pneumonia at the onset of antibiotic treatment ...67

Figure 4-4. Adjunctive dexamethasone treatment rescues ampicillin-treated mice with severe secondary pneumococcal pneumonia

Figure 4-5. Adjunctive dexamethasone therapy improves lung inflammation and pulmonary vascular permeability in mice with severe secondary pneumococcal pneumonia

Figure 4-6. Early dexamethasone treatment leads to loss of its protective activity ........71

Figure 4-7. Early dexamethasone treatment suppresses adaptive immunity

Figure 4-8. Adjunctive 2,3-diacetyloxybenzoic acid treatment improves survival of ampicillin-treated mice with secondary pneumococcal pneumonia

Figure 4-9. Early 2,3-diacetyloxybenzoic acid treatment does not worsen morbidity of influenza-infected mice. 


\section{LIST OF ABBREVIATIONS}

\begin{tabular}{|c|c|}
\hline Abs. \# & Absolute Number \\
\hline $\mathrm{ADCC}$ & Antibody-Dependent Cellular Cytotoxicity \\
\hline Amp & Ampicillin \\
\hline AMs & Alveolar Macrophages \\
\hline ANOVA & Analysis Of Variance \\
\hline APCs & Antigen-Presenting Cells \\
\hline BALF & Bronchoalveolar Lavage Fluid \\
\hline CAP & Community-Acquired Pneumonia \\
\hline CFUs & Colony Forming Units \\
\hline DABA & 2,3-Diacetyloxybenzoic Acid \\
\hline DAMPs & Damage-Associated Molecular Patterns \\
\hline DCs & Dendritic Cells \\
\hline Dexa & Dexamethasone \\
\hline DHBA & 2,3-Dihydroxybenzoic Acid \\
\hline DLNs & Draining Lymph Nodes \\
\hline EDTA & Ethylenediaminetetraacetic Acid \\
\hline GM-CSF & Granulocyte/Monocyte-Colony Stimulating Factor \\
\hline HA & Hemagglutinin \\
\hline HBSS & Hank's Buffered Salt Solution \\
\hline IFN & Interferon \\
\hline $\operatorname{Ig}$ & Immunoglobulin \\
\hline IL & Interleukin \\
\hline
\end{tabular}




\begin{tabular}{|c|c|}
\hline IMs & Interstitial Macrophages \\
\hline M1 & Matrix protein 1 \\
\hline M2 & Matrix protein 2 \\
\hline MARCO & Macrophage Receptor with Collagenous structure \\
\hline MDCK cells & Madin-Darby Canine Kidney cells \\
\hline MFI-PKH26 & Mean Fluorescence Intensity of PKH26-PCL dye \\
\hline MHC & Major Histocompatibility Complex \\
\hline MMPs & Matrix Metalloproteinases \\
\hline NA & Neuraminidase \\
\hline NEP & Nuclear Export Protein \\
\hline NETs & Neutrophil Extracellular Traps \\
\hline NK cells & Natural Killer Cells \\
\hline NLRP3 & NOD-Like Receptor family Pyrin domain containing 3 \\
\hline NLRs & NOD-Like Receptors \\
\hline NP & Nucleoprotein \\
\hline NS1 & Non-Structural protein 1 \\
\hline NS2 & Non-Structural protein 2 \\
\hline PA & RNA Polymerase Acidic subunit \\
\hline PAMPs & Pathogen-Associated Molecular Patterns \\
\hline PB1 & RNA Polymerase Basic 1 subunit \\
\hline PB1-F2 & Product of alternate open reading frame of $\mathrm{PB} 1$ gene \\
\hline PB2 & RNA Polymerase Basic 2 subunit \\
\hline PBS & Phosphate Buffered Saline \\
\hline
\end{tabular}




$\begin{array}{ll}\text { Pdm H1N1 } & \text { Pandemic influenza virus A/California/04/09 (H1N1) } \\ \text { PKH26-PCL } & \text { PKH26-Phagocyte Cell Label } \\ \text { PR8 } & \text { Influenza virus A/Puerto Rico/8/34 (H1N1) } \\ \text { PRRs } & \text { Pattern Recognition Receptors } \\ \text { PsaA } & \text { Pneumococcal surface antigen A } \\ \text { PspA } & \text { Pneumococcal surface protein A } \\ \text { RLRs } & \text { RIG I-Like Receptors } \\ \text { RLU } & \text { Relative Luminescence Units } \\ \text { RNP } & \text { Ribonucleoprotein complex } \\ \text { SBP } & \text { Secondary Bacterial Pneumonia } \\ \text { S. pneumoniae } & \text { Streptococcus pneumoniae } \\ \text { TCID50 } & \text { Median Tissue Culture Infective Dose } \\ \text { Th cell } & \text { Helper T cell } \\ \text { TLRs } & \text { Toll-Like Receptors }\end{array}$




\title{
CHAPTER 1. INTRODUCTION
}

\author{
Influenza Viruses
}

\section{Classification and Ecology}

Influenza viruses are members of the Orthomyxoviridae family. They encompass a wide variety of strains that cause significant respiratory infections in humans and various animals. Influenza viruses are antigenically classified into three genera: A, B, and C. Influenza A viruses can be further subtyped based on their two surface glycoproteins antigens: hemagglutinin (HA) and neuraminidase (NA) [1]. So far, 16 HA subtypes and 9 NA subtypes have been identified [2,3]. Standard nomenclature of influenza viruses includes virus genus, species from which it was isolated (if non-human), location where it was isolated, isolate number, and isolate year. HA and NA subtypes are added for influenza A viruses [4]. An example nomenclature of an influenza virus commonly used in the laboratory is influenza virus A/Puerto Rico/8/34 (H1N1).

The primary reservoir for influenza A viruses is aquatic birds. However, they can infect several hosts including pigs, horses, whales, seals, minks and humans. The reported ability of interspecies transmission of influenza A viruses is a major source for new pandemic strains with serious consequences on public and veterinary health [5-8].

\section{Structure of Influenza A Viruses}

Influenza A viruses are defined as enveloped RNA viruses. The viral capsid is surrounded by a lipid bilayer envelope acquired from the host cell membrane during viral particle release. The influenza A viral genome consists of 8 segmented, negative, singlestranded RNA genes. These genes encode for up to 11 known proteins, due to two splice variants of non-structural (NS) and matrix (M) genes, in addition to a product of an alternate open reading frame of polymerase basic 1 gene (PB1) in some influenza A virus strains, known as PB1-F2 [9]. Influenza viral proteins have different functions and spatial positions. Three of them are integral in the lipid envelope - HA and NA are embedded in the envelop as the major surface glycoprotein spikes, while a splice variant of $\mathrm{M}$ gene called matrix protein (M2) forms ion channels traversing the envelope [10]. This studded envelop overlays a coat composed of another matrix protein (M1). These outer layers enclose the viral core containing non-structural protein 2 (NS2) and the ribonucleoprotein complex (RNP), which consists of viral RNA segmented genes coated with nucleoprotein (NP) and RNA-dependent RNA polymerase. The viral RNA polymerase is composed of 3 subunits: 2 polymerase basic subunits (PB1 and PB2) and 1 polymerase acidic subunit (PA) (Figure 1-1) [4, 11]. Recently, a novel viral protein, termed PA-X, was identified as the product of the second open reading frame of PA gene which can be accessed through ribosomal frameshifting [12]. 

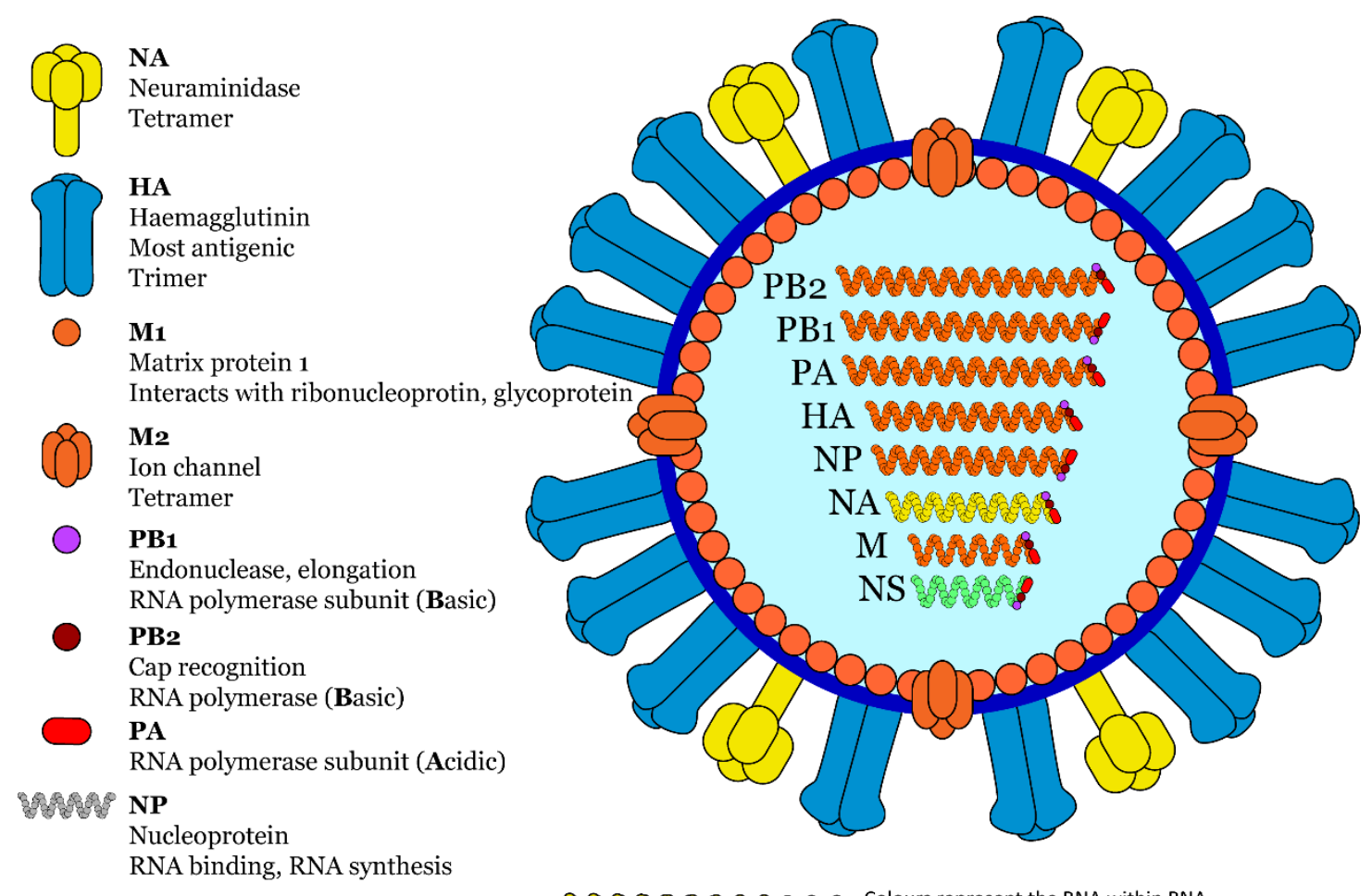

Colours represent the RNA within RNA
encapsidated segments enclosed by NP.

\section{Figure 1-1. Influenza A virus structure}

Reprinted with permission from Dr. Ian M. Mackay. Virology Down Under, http://www.uq.edu.au/vdu/ (Last accessed on September 5, 2013) [11]. 


\section{Replication Cycle}

The influenza A virus replication cycle starts by virus attachment through HA spikes on the viral envelope to terminal $\mathrm{N}$-acetylneuraminic (sialic) acid moieties on the host cell surface. The type of linkage of these terminal sialic acid moieties to galactose determines viral tropism to host cells, as HA may have preferential binding to sialic acid receptors with either $\alpha-2,3$ or $\alpha-2,6$ linkages [13]. Generally, HA of human influenza viruses binds preferentially to sialic acid with $\alpha-2,6$ linkage, which is more abundant on human tracheal epithelial cells $[14,15]$, whereas HA of avian influenza viruses binds to sialic acid with $\alpha-2,3$ linkage $[13,16,17]$, which is abundant on gut and respiratory epithelial cells of ducks [18].

Upon HA binding to the host cell sialic acid residues, receptor-mediated endocytosis occurs. The virus enters the host cell in a clathrin-coated endosome, whose acidic $\mathrm{pH}$ triggers a conformational change of HA leading to fusion of the viral and endosomal membranes, followed by release of nucleocapsid into the cell cytoplasm [19]. In addition, M2 channels in the viral envelope pump hydrogen protons into the viral core. This internal acidification process disrupts internal protein-protein interactions, causing the M1 protein to dissociate from RNP and eventually leading to viral uncoating and release of viral RNP into the cell cytoplasm $[4,20]$.

After their cytosol release, viral RNPs are actively translocated to the host cell nucleus through nuclear localization signals on internal viral proteins [21]. Upon entering the nucleus, transcription and replication processes of the viral genome start. Viral RNA serves as a template for mRNA and complementary positive-stranded RNA (cRNA) synthesis. Next, viral mRNA is exported out of the nucleus to be translated into viral proteins. cRNA is amplified into genomic viral RNAs, which associate with internal viral proteins, forming newly synthesized viral RNP segments. Nuclear export of the progeny viral RNP segments into the cytoplasm is mediated by M1 and nuclear export protein (NEP)/NS2 proteins $[4,21]$. Then, they are transported to the cell membrane - the site of viral RNP segments packaging and assembling of the new viral particles. Growing evidence suggests that the packaging process of eight unique viral RNP segments is a selective, yet unclear, process $[22,23]$. M1 protein is accumulated at the cytoplasmic side of the lipid bilayer which facilitates viral budding. Finally, NA protein through its sialidase activity cleaves terminal sialic acid residues on both the host cell surface and the viral envelope to facilitate the release of new viral particles, and prevent aggregation of viral particles, respectively [4, 24].

\section{Pathogenesis in Humans}

Influenza and pneumonia remain among the leading causes of deaths worldwide. Influenza A viruses can cause acute respiratory viral infections that can be easily transmitted among humans by inhalation of respiratory droplets. They have been circulating since at least the 16th century with a unique ability to cause recurrent annual epidemics [25]. Moreover, upon emergence of a novel influenza virus, by which the total 
population has not been attacked before and consequently people have no specific adaptive immunity against, this novel virus spreads quickly among different age groups and healthy young individuals of the population with rapid transmission to several regions worldwide leading to influenza pandemics. Several such pandemics occurred in the 20th century causing millions of deaths worldwide. The specter of influenza pandemics still imposes a continuous threat to world public health in the 21 st century, after the recent swine H1N1 influenza pandemic emerged in 2009-2010 and caused more than 284,000 deaths globally in the first year [26, 27].

In humans, acute influenza infections cause diseases of variable severity, ranging from mild upper respiratory tract infections to acute respiratory distress syndrome and death. Several factors count for the variability in infection outcomes. Therefore, it is crucial to understand factors related to the virus and the host which contribute to increased morbidity and mortality.

\section{Viral Virulence}

Viral virulence is a complex phenomenon which involves several factors, including viral tropism, transmissibility, and replication efficiency [28]. It is a multigenic trait, resulting from the collective contribution of different viral genes and proteins into viral pathogenicity. However, certain viral genes from some highly virulent influenza strains may increase virulence if put into the genetic background of another strain. For example, studies of recombinant strains produced by a reverse genetics system using HA and NA genes or even a single HA gene from the highly virulent 1918 pandemic influenza strain plus the genetic background of another strain increased the recombinant virus virulence in mice $[26,29,30]$. Strikingly, just one mutation in a certain gene of some strains may greatly affect their virulence [26].

Circulating highly virulent influenza viruses pose a continuous threat of a new serious influenza pandemic. An example of recently emerged highly pathogenic influenza strains that have pandemic potential is the avian H5N1 influenza viruses, which caused high fatality rate of about $60 \%$ in the identified infected humans [31]. However, they have poor human-to-human transmission and remain endemic in poultry populations, mostly in Southeast Asia [6]. Nonetheless, recent studies showed that these highly pathogenic viruses may naturally adapt and acquire efficient human-to-human transmission, posing a great threat to humans [32,33]. With currently limited spread among humans, these highly pathogenic avian viruses have given only minor contribution to global influenza-associated morbidity and fatality with 378 deaths among cumulative 637 cases of infection from 2003 until August 2013 [34].

In general, it has been observed that influenza viral infections alone do not account for most mortality during seasonal, epidemic, or pandemic influenza periods. Instead, complications during influenza infections were found as the main reasons for increased influenza-associated mortality. For instance, people with certain comorbidities are considered high-risk populations during influenza epidemics and pandemics, and 
antiviral therapy is highly recommended for them. This high-risk category includes those with weakened immunity, such as infants and children less than 5 years old, as well as the elderly, pregnant women, and people with serious respiratory or cardiovascular diseases. Additionally, bacterial super-infections remain a serious complication among all influenza-infected hosts and were found to complicate many severe cases of influenza infections and significantly contribute to fatalities during influenza epidemics and pandemics $[35,36]$.

Despite its importance, viral virulence is primarily a threat to immunologically naïve hosts. Therefore, highly pathogenic viruses may only have minor effects on healthy individuals with previous exposure or those who were vaccinated against closely related viral strains. Thus, host immunity is another key factor in virus-host interactions and influenza infection outcomes.

\section{Host Immune Responses}

Host immunity can be classified into two branches: innate non-specific immunity and adaptive specific immunity. Once the influenza virus enters its target cell and starts replication in the respiratory tract, diverse host immune responses launch, starting with initial responses of infected cells and progressing to more advanced and specific immune responses. To initiate host immune responses, a pathogen or its associated pathogenic activity must be detected. Detection is performed by receptors in the host cells called pattern recognition receptors (PRRs). These receptors play key roles in the innate immune response to infections by a wide range of microorganisms, including influenza viruses. PRRs are activated when they detect and bind to certain conserved microbespecific motifs or molecules, known as pathogen-associated molecular patterns (PAMPs). PPRs can also detect certain host endogenous molecules released during tissue damage called damage-associated molecular patterns (DAMPs). PRRs are composed of diverse sets of receptors including Toll-like receptors (TLRs), nucleotide oligomerization domain (NOD)-like receptors (NLRs), retinoic acid inducible gene-I (RIG-I)-like receptors (RLRs), and cytosolic DNA and viral RNA sensors. Some of them are expressed on the surface of host cells, including TLR1, TLR2, TLR4, TLR5, and TLR6, making them suitable for detecting extracellular pathogens and certain DAMPs. However, other PRRs, such as TLR3, TLR7, and TLR9, and RLRs, present intracellularly in the endosomes or host cell cytosol to detect intracellular pathogens $[37,38]$.

Activation of various PRRs during influenza virus replication triggers certain signaling pathways in the detecting host cells, eventually leading to synthesis of different cytokines and chemokines (Figure 1-2) [28]. Infected respiratory epithelial cells and pulmonary macrophages are the first cells to detect viral replication. Three main categories of PRRs can detect influenza virus infection: TLRs, such as TLR3 and TLR7, which bind to viral double-stranded RNA and single-stranded RNA, respectively; RIG-I receptors which recognize newly synthesized viral RNAs [39]; and NLR receptor family pyrin domain containing 3 (NLRP3), which is activated by influenza viral RNA or M2 ion channel activity during influenza infection [40-42]. Some of the cytokines produced 


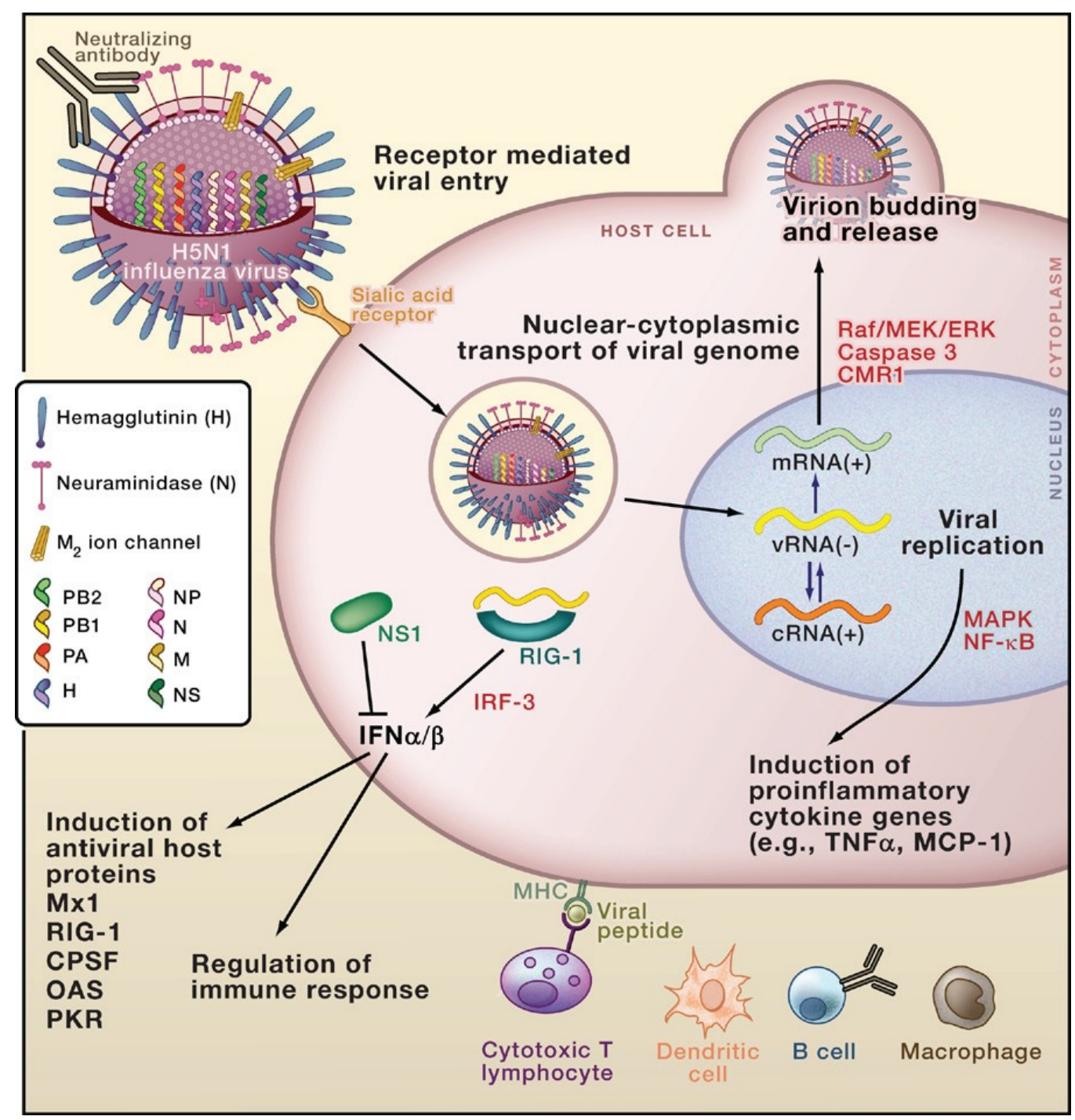

Figure 1-2. Molecular basis of immune responses at site of influenza infection

Reprinted with permission from Elsevier. Salomon R, Webster RG. The influenza virus enigma. Cell 2009; 136:402-10 [28]. 
early have strong antiviral activities, such as type I interferons (IFN $\alpha / \beta)$ and type III IFN (IFN $\lambda$ ), which protect nearby non-infected cells against influenza infection by inducing synthesis of various antiviral proteins. In addition, they stimulate dendritic cells to enhance antigen presentation for $\mathrm{T}$ cells, thereby facilitating adaptive immune response development. Inflammatory response may be blocked in the first 2 days after influenza infection but then pro-inflammatory cytokines and chemokines are suddenly released signaling recruitment and activation of various circulating immune cells to the site of infection (Figure 1-2) [43-45].

Several types of host immune cells are recruited to the infection site with different kinetics and different performance characteristics but temporally integrated functions. Extravasation of innate immune cells, such as neutrophils, natural killer cells, exudate monocytes and macrophages, and dendritic cells start in the first few days after infection. Then, activated influenza-specific adaptive immune cells are recruited with more targeted functions in fighting influenza virus infection. After viral clearance, these influenzaspecific adaptive immune cells play a critical protective role against future re-infections by the same or closely related strains by establishing influenza-specific memory immunity (Figure 1-3) [46].

Because of their spatial position on the mucosal surface of alveolar epithelium, resident alveolar macrophages (AMs) are the first innate immune cells to encounter influenza virus upon its spread to the lower respiratory tract. They detect early virus replication and become activated, releasing several pro-inflammatory cytokines and chemokines. Thereby, they signal recruitment of innate immune cells that participate in controlling viral replication during the inflammatory phase of infection. Different influenza A virus strains have different abilities to infect human and murine AMs [47]. However, infected AMs produce low virus yield. AMs were thought for a long time to be a central activator for the induced inflammatory cytokine storm when they are infected by the highly pathogenic H5N1 influenza viruses [43]. In contrast, a recent study strikingly showed that human AMs, and not blood monocyte-derived macrophages, can release only small amounts of pro-inflammatory cytokines after being infected by different influenza viruses, including highly pathogenic H5N1 viruses [48]. These data are relatively relevant with the observed immunosuppressive nature of AMs [49, 50]. In contrast, another study showed that influenza infections of isolated human AMs induce robust release of different cytokines and chemokines, including type I IFN, while downregulating expression of certain receptors, such as, macrophage scavenger receptor 1 and CD36 [51].

Dendritic cells (DCs) are generally professional antigen-presenting cells (APCs) that bridge innate and adaptive immunity. Their activation during influenza infection enhances the uptake, processing, and presentation of influenza antigens. Thereafter, some DCs migrate to the draining lymph nodes (DLNs) where they present influenza antigenic epitopes on either major histocompatibility complex (MHC) class I and II molecules to $\mathrm{CD}^{+} \mathrm{T}$ cells and $\mathrm{CD} 4^{+}$helper $\mathrm{T}(\mathrm{Th})$ cells, respectively. This step is crucial for the initiation of adaptive immune responses. In mouse models of influenza infection, several subsets of DCs, such as migratory $\mathrm{CD} 103^{+} \mathrm{CD} 11 \mathrm{~b}^{-}$conventional DCs and CD $11 \mathrm{~b}^{+} \mathrm{DCs}$, 


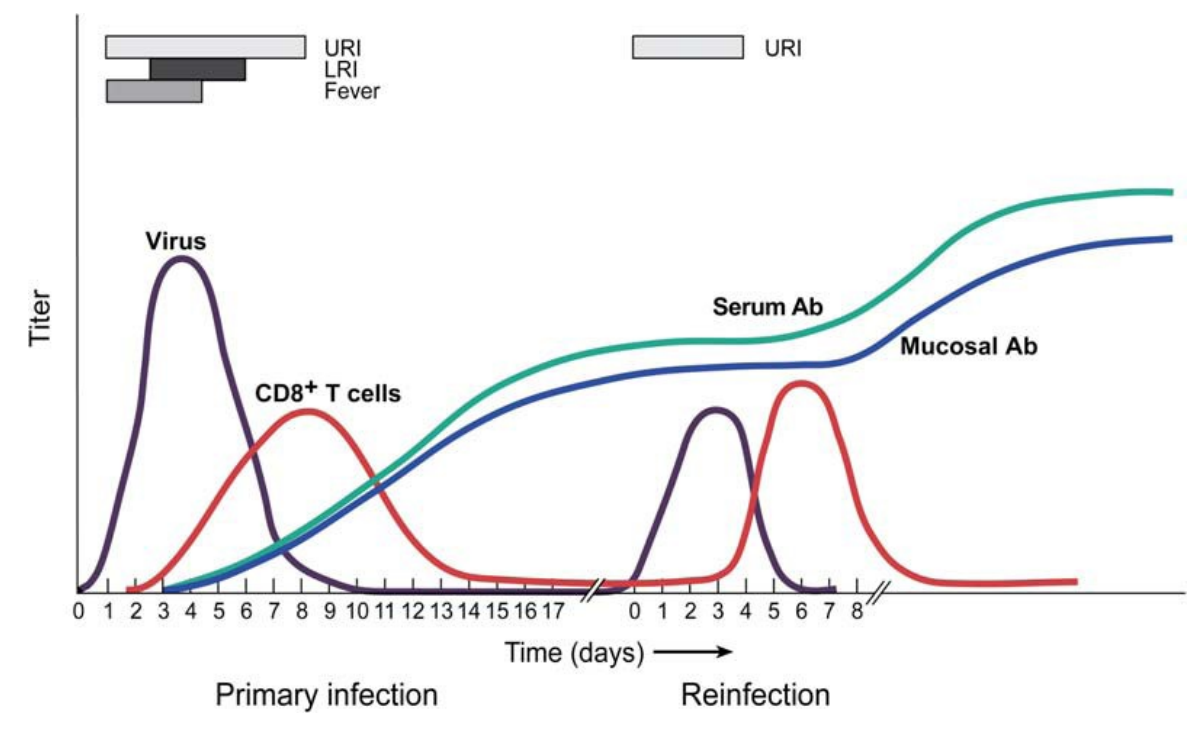

Figure 1-3. Kinetics of adaptive immune responses during primary and secondary influenza infections

Reprinted with permission from Elsevier. Subbarao K, Murphy BR, Fauci AS.

Development of effective vaccines against pandemic influenza. Immunity 2006; 24:5-9 [46]. 
have been identified to be crucial for mounting efficient adaptive immunity against influenza viruses. They carry influenza antigens from the site of infection and migrate to the DLNs to prime and activate expansion of influenza-specific T cells. In addition, monocyte-derived DCs may be necessary to sustain effector T cells in the lungs for viral clearance [52]. In contrast, some inflammatory DCs may also cause detrimental immunopathology in the lungs during lethal influenza infection [53-55].

Natural killer (NK) cells are also important innate immune effector cells. They can directly kill influenza-infected cells after triggering their cytotoxicity receptors, such as NKp44 and NKp46, by binding to influenza HA proteins expressed on influenzainfected cells. In addition, antibody-dependent cellular cytotoxicity (ADCC) is another mechanism in which NK cells lyse antibody-bound influenza-infected cells after binding their CD16 receptor (FcyRIII) to the Fc portion of the bound antibodies [43, 56].

Neutrophil influx is a hallmark of the early innate immune response to influenza infection. Neutrophils significantly participate in limiting influenza viral replication in the early inflammatory phase of infection. Furthermore, they may also facilitate the initiation of adaptive immune responses. They release different cytokines and chemokines that signal recruitment and activation of DCs. In addition, they may modulate the activities of T cells [57-59]. On the other hand, if neutrophil influx and their pro-inflammatory functions are not tightly controlled, they may remarkably contribute to lung immunopathology and increased morbidity and lethality [60, 61].

Exudate monocytes and macrophages also participate in the inflammatory innate immune response to influenza infection. Their infiltration is induced by different proinflammatory cytokines and chemokines released from infected cells and virus-detecting immune cells, such as pulmonary macrophages and DCs. This process is mainly CCR2dependent preferentially recruiting $\mathrm{CCR} 2{ }^{+}$mononuclear phagocytes. They contribute in limiting viral spread via phagocytosis of infected apoptotic bodies. However, they may also become destructive causing immunopathology by enhancing the release of proinflammatory cytokines and tissue-damaging species, such as tumor necrosis factor- $\alpha$, interleukin-6 (IL-6), and nitric oxide [54]. Thus, prophylactic CCR2-antagonist treatment significantly attenuated lung immunopathology without affecting viral clearance and improved survival in a mouse model of severe influenza infection [62].

A few days after launching the acute inflammatory innate immune response, the influenza infection becomes more efficiently controlled by influenza-specific B cells and $\mathrm{CD}^{+} \mathrm{T}$ cells, both effectors of antibody-mediated (humoral) and cell-mediated (cellular) adaptive immunity (Figure 1-3 and Figure 1-4). They are initially activated in the secondary lymphatic tissues, such as the DLNs, after recognizing influenza antigenic epitopes presented on MHC class I and II molecules on the surface of activated APCs expressing co-stimulatory molecules with the help of activated $\mathrm{CD} 4{ }^{+} \mathrm{Th}$ cells. After activation and clonal expansion, influenza-specific $B$ cells and $T$ cells migrate to an influenza infection site under chemoattractant gradient. Specific T cell receptors on the surface of $\mathrm{CD}^{+}$cytotoxic T cells recognize influenza antigenic epitopes presented on MHC class I molecules on influenza-infected host cells. They kill infected cells via 
a Antibody-mediated immunity
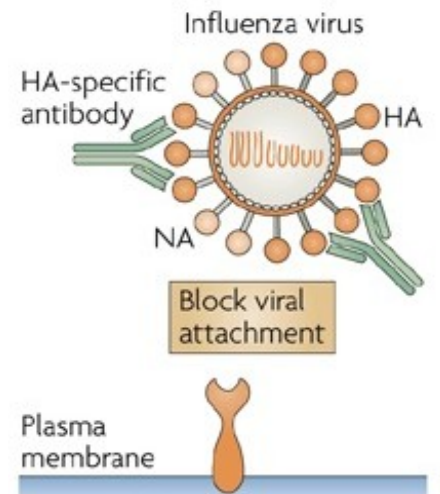

Sialic-acid receptor

Epithelial cell

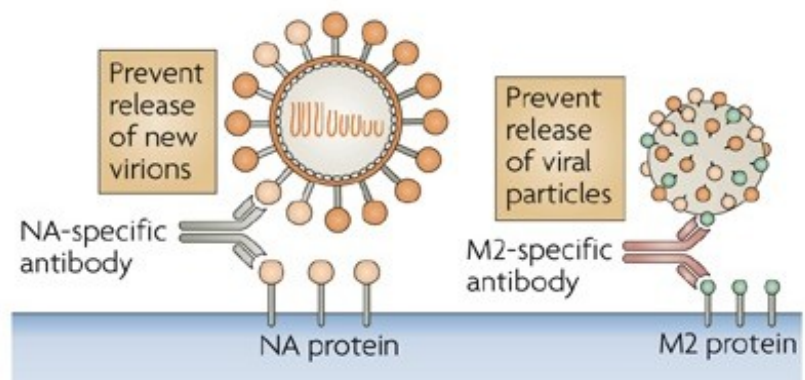

b Cell-mediated immunity

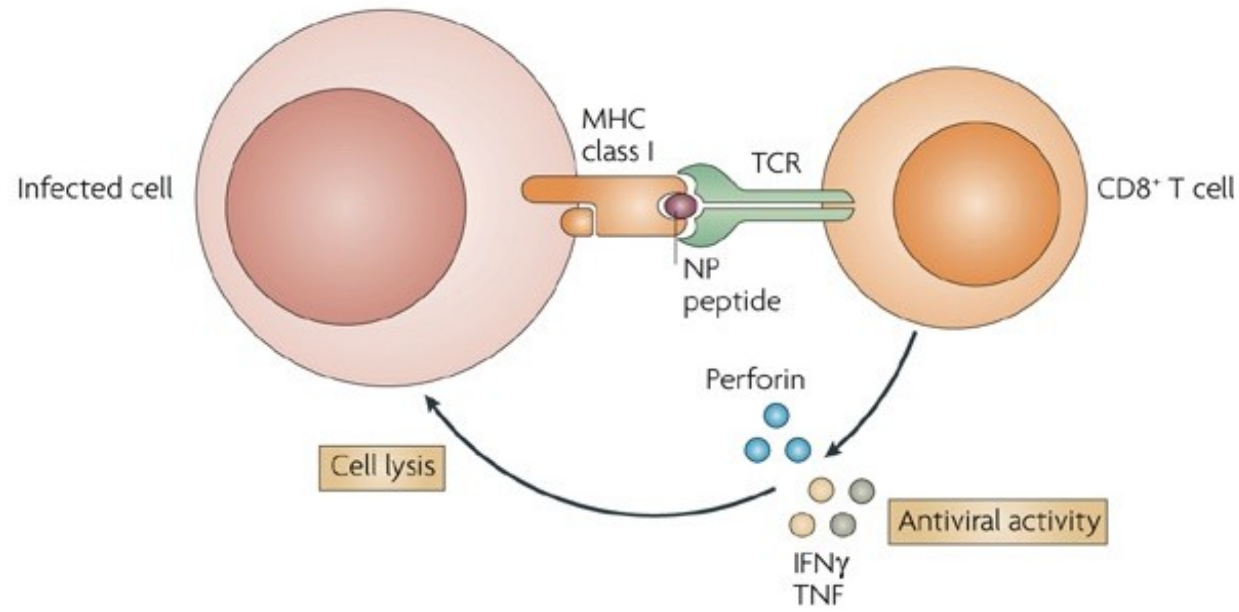

Figure 1-4. Adaptive immune response against influenza virus infection

Adapted with permission from Nature Publishing Group. Subbarao K, Joseph T. Scientific barriers to developing vaccines against avian influenza viruses. Nat Rev Immunol 2007; 7:267-78 [63]. 
perforin-mediated cytolysis and granzymes and FAS/FASL-induced apoptosis. In addition, plasma cells secrete antibodies targeting several influenza antigenic epitopes, especially surface antigens HA, NA, and M2, which neutralize the infectivity of influenza viral particles (Figure 1-4). Furthermore, influenza-specific antibodies bound to viral particles facilitate their clearance and phagocytosis by binding to Fc receptors of various phagocytes. They also bind to influenza antigens expressed on the surface of infected cells and activate their killing by the NK cell ADCC mechanism [43].

After viral clearance, some virus-specific B cells and T cells differentiate into memory cells. They reside mainly in some lymphoid organs and structures, such as the spleen and inducible bronchus-associated lymphoid tissue, establishing specific memory. Their protective functions rely on their ability to launch faster specific adaptive immune responses against future infections by the same or closely related influenza virus strains (Figure 1-3).

Indeed, efficient innate and adaptive host immune responses are required to inhibit viral replication and facilitate viral clearance. On the other hand, excessive immune responses will have deleterious effects and cause collateral lung tissue damage. Therefore, respiratory epithelial cells and other effectors play important roles to restore lung homeostasis during influenza infections and to prevent the damaging effects of robust host immune responses. For example, lung epithelial cells are highly expressing inhibitory molecule CD200 which inhibits inflammatory activation of recruited macrophages in addition to AMs by binding to CD200 receptor. Moreover, lung epithelial cells constitutively express latent TGF- $\beta$ which was shown to maintain pulmonary immunological homeostasis under steady state. It can get activated by influenza NA activity. In vivo blockage of TGF- $\beta$ during influenza infection was shown to increase disease morbidity, implying its potential immunomodulatory activity $[55,64$, $65]$.

\section{Immune Evasion by Influenza Viruses}

To be successful against the elaborate host immunity, influenza viruses use a variety of strategies to evade host immune responses. Several viral proteins have been shown to antagonize antiviral innate immune responses and alter the type I IFN response. For example, functional influenza NS1 protein can interfere with RIG-I receptor signaling at several stages. Furthermore, NS1 may also impair DC maturation, thereby indirectly limiting adaptive immunity development. Other viral proteins, including PB2, PB1-F2, PB1, and PA also have antagonistic activities against the type I IFN response $[56,66,67]$.

More strikingly, influenza viruses have a unique ability to change their genome. Thus, they can evade host adaptive immunity post continuous threats to humans, even to a vaccinated populations. Influenza viruses can undergo two types of antigenic changes: antigenic shift and antigenic drift. Antigenic shift leads to major changes in the viral genome through a reassortment process between genes of different influenza virus strains 
in a cell simultaneously infected with two of more strains. These reassortment events mostly take place in avian or swine reservoirs, resulting in the emergence of a new strain that can be transmitted to and circulate among hosts of the same species or transmitted to humans, causing recurrent epidemics or global pandemics. In contrast, antigenic drift results from mutations in viral genes due to the lack of proofreading activity of influenza viral RNA polymerase. This leads to minor changes in the antigenic epitopes of the virus, mostly in the surface antigens HA and NA. These random mutations are preserved under selective pressure of the host influenza-specific adaptive immunity, generating escape mutant strains $[43,56]$.

\section{Streptococcus pneumoniae}

\section{History}

Streptococcus pneumoniae, also commonly known as pneumococcus, is among the first discovered bacterial pathogens causing human diseases. In 1881, pneumococcus was independently isolated by George M. Sternberg in the United States and Louis Pasteur in France. They described lancet-shaped pairs of spherical bacteria in human saliva. They showed that a new septicemic disease developed rapidly after injecting rabbits with human saliva, and recovered the same bacteria from infected rabbit blood [68].

After several nominations, pneumococcus was named Diplococcus pneumoniae in 1920 due to its observed morphology as pairs of cocci, and its commonly reported ability to cause pneumonia in humans [69]. In 1974, it was renamed Streptococcus pneumoniae, as it grows in chains in liquid media [68].

Since the discovery of pneumococci in the 19th century, they have been heavily investigated and played a central role in several breakthrough discoveries. For example, studies on pneumococci expanded our understanding of the concept of host humoral immunity and the vaccine production, and facilitated the discovery of DNA as genetic material which gave rise to Molecular Genetics [68, 70, 71]. Moreover, it accelerated the development and use of penicillin and related antibiotics after they showed efficacy in treating pneumococcal infections $[72,73]$.

\section{Identification}

Pneumococci are Gram-positive bacteria belonging to Streptococcus genus which are catalase-negative and can ferment glucose to lactic acid. However, pneumococci produce alpha-hemolysis when grown on blood agar plates under $5 \% \mathrm{CO}_{2}$ conditions. Identification through special biochemical tests is based on pneumococcal ability to hydrolyze inulin, and its sensitivity to optochin and bile salt [74]. Further classification is based on serotyping. The pneumococcus is surrounded by a polysaccharide capsule that 
is highly antigenic, inducing specific serum antibodies. Antigenic classification of the pneumococcal capsule is performed by a capsular swelling test or quellung reaction, which allows identification of more than 90 capsular serotypes [75-77].

\section{Virulence Factors}

Pneumococci reside on the mucosal surface of the upper respiratory tract of asymptomatic carriers who are the primary reservoir of $S$. pneumoniae, from which it can be transmitted to other individuals by inhalation of respiratory droplets. Alternatively, it can spread locally to cause upper or lower respiratory tract infections. It can also invade the blood, causing septicemia and meningitis.

This clinically challenging pathogen has a diverse armament of virulence factors helping it switch from the colonization state to a pathogenic nature that causes respiratory tract infections or septicemia (Figure 1-5). One of the key virulence factors is the polysaccharide capsule, which resists phagocytosis in the absence of capsule-specific antibodies. It acts as a shield, preventing complement $\mathrm{C} 3 \mathrm{~b}$ deposition on the bacterial cell wall, thereby protecting the bacteria against opsonin-mediated phagocytosis. It also decreases the trapping of pneumococci in neutrophil extracellular traps [76]. Differences in the capsular structure led to the identification of more than 90 capsular serotypes with varying degrees of virulence [76].

The pneumococcal cell wall has characteristic teichoic acid residues attached to the peptidoglycan in addition to lipoteichoic acid attached to the bacterial cell membrane. Both moieties have phosphorylcholine, which can bind to choline-binding receptors on the surface of human cells. In addition, there are several types of pneumococcal surface proteins that play important roles in bacterial adherence, colonization, in vivo growth, and virulence. Among these surface proteins are choline-binding proteins, including pneumococcal surface proteins A and C (PspA and PspC); metal-binding proteins, including pneumococcal surface antigen $\mathrm{A}$ (PsaA), pneumococcal iron acquisition $\mathrm{A}$ (PiaA), and pneumococcal iron uptake A (PiuA); and pneumococcal adhesion and virulence A (PavA) [76].

Pneumococci are also characterized by the expression of autolysins or cell wall hydrolases that induce natural pneumococcal cell wall lysis, during the stationary phase of growth and facilitate penicillin-induced lysis. The major autolysin, LytA, has been shown to be important for pneumococcal virulence, partly by facilitating the secretion of another key virulence factor - pneumolysin. In addition, LytA may be released to lyse neighboring non-competent pneumococcal cells in a fratricidal manner [78]. By doing so, it facilitates genetic exchange between naturally competent pneumococcal cells that easily take up and incorporate DNA by homologous recombination. Furthermore, autolysin facilitates the release of pneumococcal cell wall fragments and teichoic acids that trigger inflammatory reactions and may interfere with phagocyte-mediated elimination of live pneumococci [76, 79]. 


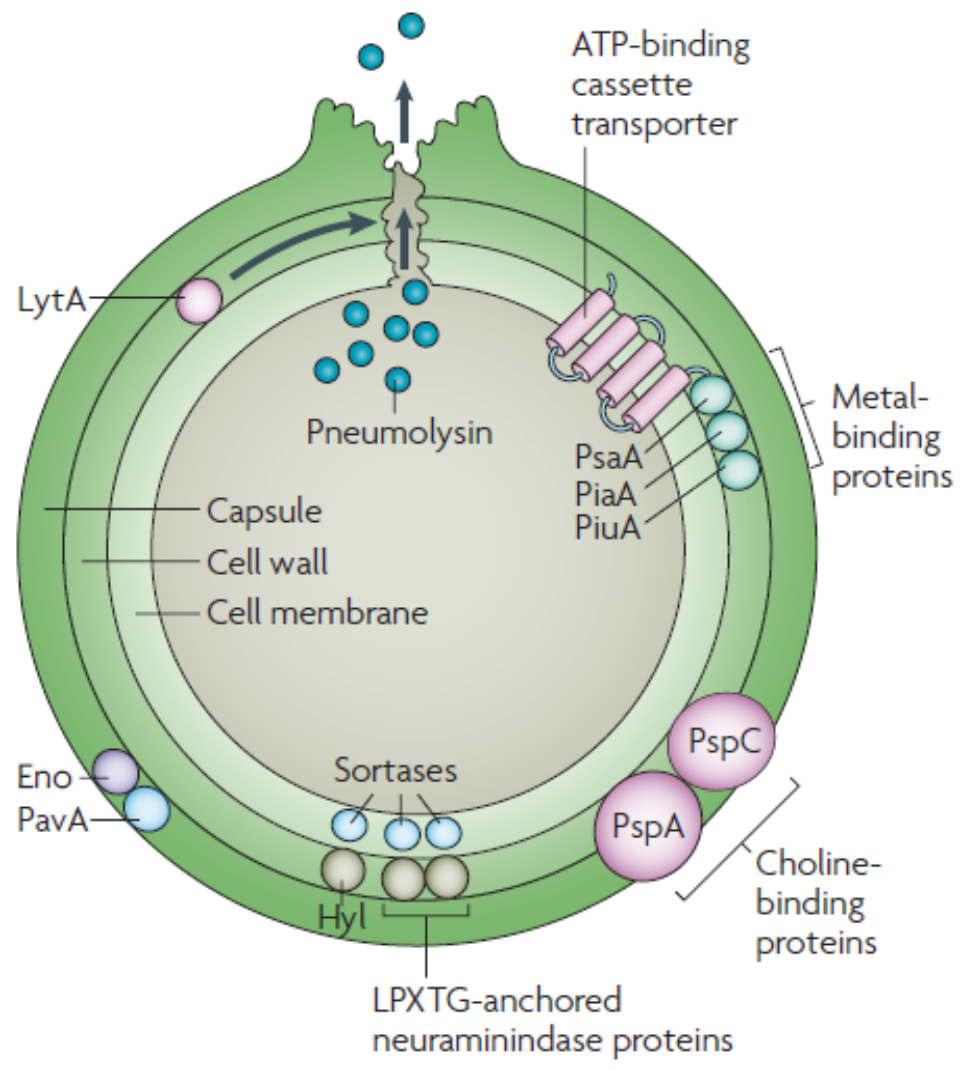

Figure 1-5. Streptococcus pneumoniae structure and virulence factors

Reprinted with permission from Nature Publishing Group. Kadioglu A, Weiser JN, Paton JC, Andrew PW. The role of Streptococcus pneumoniae virulence factors in host respiratory colonization and disease. Nat Rev Microbiol 2008; 6:288-301 [76]. 
Pneumolysin is another key virulence factor with diverse functions. When secreted in high enough concentrations, it acts as a cholesterol-dependent, pore-forming cytotoxin through oligomerization in the host cell membrane. At sub-lytic concentrations, it can modulate target cell functions, such as inhibiting ciliary beating of respiratory epithelial cells, and augmenting the synthesis of inflammatory cytokines. It may also increase pneumococcal virulence by aggravating inflammatory responses during pneumococcal infections [76]. Recently, it was shown that pneumolysin triggers IL-1beta and IL-18 secretion through NLRP3 inflammasome activation, which probably mediates host resistance against pneumococcal infections [80].

Moreover, pneumococci can secrete 3 neuraminidase proteins (NanA, NanB, and $\mathrm{NanC}$ ) that are important for pneumococcal in vivo growth. All strains express NanA, most strains express NanB, and some strains express NanC [76, 81]. They cleave terminal sialic acid residues from host cell surface glycoproteins or soluble host proteins, such as lactoferrin. Thereby, they help reveal receptors for bacterial adherence on the host cell surface. They can also secrete a zinc metalloprotease called IgA1 protease that targets host mucosal IgA1 [76].

\section{Pneumococcal Diseases}

S. pneumoniae is a serious human bacterial pathogen found to be the most common causative agent of pneumonia leading to hospitalization. Pneumococci can spread locally from the nasopharynx to cause otitis media or sinusitis, or to the lungs, probably by microaspiration, causing pneumonia. Beyond the lungs, pneumococci can also invade epithelial barriers to the blood circulation, causing bacteremia, or severe invasive infections, such as meningitis, with high mortality rates [82, 83].

During the transition of pneumococci from nasopharyngeal colonization to invasive infections, they undergo phase variation and modulate the expression of several virulence factors to become adapted to various microenvironments within the host. Among these switches is capsule formation. Maximal capsule expression is critical for systemic infections to help escape from various host humoral immunity and phagocytic effectors. Therefore, pneumococci isolated from blood are highly encapsulated. In contrast, during nasopharyngeal colonization, pneumococci have minimal capsule expression to facilitate exposure of pneumococcal adhesins for better attachment to respiratory epithelial cells [76].

\section{Innate Immunity}

Host immune responses during pneumococcal infections are multifaceted as pneumococci are encountered by several lines of host defense, including innate nonspecific host defense lines, such as mucociliary clearance, complement activation, neutrophils and macrophages. In addition, specific adaptive immunity is triggered, generating protective humoral and cellular immunity against pneumococcal infections. 
Several key protective immune effectors were highlighted by observing hosts with genetic deficiencies in certain immunity components and showing high susceptibility to recurrent pneumococcal infections. Among them are the patients with deficiency in some components of the complement system, such as $\mathrm{C} 3$, the central component of complement activation pathways. Likewise, in old humans or aged mice, increased susceptibility to pneumococcal pneumonia is associated with decreased inflammatory immune responses, which may stem from the dysfunction of various TLRs in the lungs $[84,85]$.

The complement system is a humoral component of innate immunity involving more than 30 inactive serum and membrane-bound proteins. They are activated in a cascade manner through 3 pathways and release effector molecules with diverse functions. Complement activation pathways include the classical pathway, which is activated by microbial antigen-antibody complexes or by acute phase proteins bound to microbial surface, and the alternative pathway, which is constitutively turned on but to a minor extent due to host cell-bound inhibitors. In contrast, activation of the alternative pathway is amplified on foreign microbial surfaces due to absence of these inhibitors. The third type is the lectin pathway, which is activated upon binding host mannosebinding lectin to carbohydrates on microbial surfaces. The activation outcomes by any pathway comprise 3 major functions: deposition of certain cleaved components on microbial surface functioning as opsonins, which enhance phagocytosis; chemotactic functions for neutrophils and other inflammatory immune cells; and direct killing of the microbe by membrane attack complexes which form pores in the microbial cell surface [86].

During pneumococcal infections, the classical pathway is the dominant pathway for complement activation and innate immunity against pneumococci [87]. It can be activated after binding host natural IgM antibodies or acute phase proteins, such as $\mathrm{C}$ reactive protein, to the pneumococcal surface $[84,88]$. Strikingly, secreted pneumolysin was found to activate the classical pathway in an antibody-independent manner.

However, this phenomenon was shown to contribute to pneumococcal virulence by activating and consuming the complement away from the pneumococcal surface, thereby preventing deposition of activated complement components on the pneumococcal surface [89].

Respiratory epithelial cells are the first cells to detect pneumococcal colonization and infection. They and other local immune cells express a wide range of PRRs. Diverse PRRs are triggered during pneumococcal infections and signal activation and chemotaxis of host immune cells by inducing the synthesis of different pro-inflammatory cytokines and chemokines. Among them, TLR2, expressed on the host cell surface as heterodimers with either TLR1 or TLR6, is activated by several pneumococcal-derived lipoproteins, such as lipoteichoic acid. Experimental genetic deletion of TLR2 in mice increased pneumococcal virulence by impairing efficient innate immune responses [84]. In addition, some cytosolic PRRs, such as NOD1 and NOD2, are activated by the cell wall of internalized pneumococci [90]. TLR9 is an intracellular endosomal PPR that can recognize pneumococcal DNA which is released from spontaneously lysed pneumococci, 
and enhance bacterial phagocytosis [91] Additionally, pneumolysin augments the induction of various inflammatory cytokines and can activate NLRP3, which is important for rapid pulmonary clearance of pneumococci in mice [80].

Surfactant proteins are highly synthesized in the lungs to prevent the collapse of alveoli. Among them, SP-D and SP-A have innate immune functions during pulmonary pneumococcal infections by binding to pneumococci and enhancing their phagocytosis [92].

Cellular innate immune effectors are crucial for early control of the replicating pneumococci through phagocytosis and intracellular killing. Among them, resident AMs are the critical first line of cellular innate immunity protecting lungs against invading bacterial pathogens, including pneumococci [93]. Resident AMs are characterized by high phagocytic capacity and play a key role in immunological homeostasis and maintenance of the anti-inflammatory milieu in the lungs under steady-state conditions $[94,95]$. Artificial depletion of AMs in mice enhanced pneumococcal replication in the lungs $[93,96]$.

Although pneumococci can resist complement-mediated opsonophagocytosis via its polysaccharide capsule, the dynamic surface of resident AMs is characterized by a diverse repertoire of surface non-opsonic receptors facilitating efficient phagocytosis of encapsulated pneumococci. This repertoire includes class A scavenger receptors, such as $\mathrm{SR}-\mathrm{AI} / \mathrm{II}$ and macrophage receptor with collagenous structure receptor (MARCO), as well as C-type lectin mannose receptor, which can bind to the pneumococcal capsule, thereby playing an important role in early pneumococcal clearance from murine lungs $[97,98]$. Recently, CD36, a scavenger receptor expressed on the surface of AMs and respiratory epithelial cells, was shown to downregulate the early inflammatory response while enhancing bacterial phagocytosis in an animal model of pneumococcal pneumonia [99]. In addition, downregulation of MARCO receptor or impaired AM functions by TLR desensitization after influenza infection may permit respiratory pneumococcal outgrowth in animal models of secondary pneumococcal pneumonia $[100,101]$.

Inside AMs, phagocytosed pneumococci are destroyed by the conventional intracellular killing mechanisms which involve lysosomal fusion and phagosome maturation, accompanied by degradative action of various lysosomal enzymes and bacterial cell damage by the NADPH oxidase-catalyzed oxidative burst. However, when the intracellular killing capacity of AMs is exhausted, lysosomal permeabilization and activation of cathepsin D take place leading to AM apoptosis with consequent killing of internalized bacteria. [102].

In sub-clinical infections, efficient pulmonary clearance of pneumococci by resident $\mathrm{AMs}$ is achieved preventing severe inflammatory sequelae. In contrast, when the pneumococcal burden overwhelms the phagocytic capacity of resident AMs, secretion of pro-inflammatory cytokines and chemokines is increased, mainly from the respiratory epithelial cells, inducing recruitment of neutrophils, the second line of cellular innate immunity against pneumococci [103]. Neutrophils or polymorph nuclear leukocytes are 
short-lived circulating immune cells and are the first cells to accumulate at sites of pneumococcal infections or colonization. They become the dominant inflammatory immune cells in the pneumonic lungs [104]. In addition, $\gamma \delta$ T cells may play an important role in the host defense against pulmonary pneumococcal infections by promoting neutrophil influx in the lungs [105].

To kill pneumococci, neutrophils use a diverse armament involving phagocytosis and intracellular bacterial killing by a combination of respiratory burst and non-oxidative mechanisms. Activation of NADPH oxidase and myeloperoxidase, the primary arm of the oxidative mechanism, leads to generation of various oxidizing species with potent bactericidal activities. In addition, neutrophils are loaded with intracellular granules that release several antimicrobial molecules, such as $\alpha$-defensins, cathepsin $\mathrm{G}$, lysozyme, lactoferrin, and neutrophil elastase, upon fusion with the phagosome [106].

Neutrophils are also characterized by a unique mechanism of extracellular bacterial killing. This mechanism involves the release of neutrophil DNA strands loaded with antimicrobial molecules that form neutrophil extracellular traps (NETs) with potent bactericidal activities. Although pneumococci are captured within NETs, they can escape NET-mediated killing by secretion of bacterial endonuclease. In addition, the pneumococcal capsule can decrease bacterial trapping in NETs. Likewise, increasing positive charges over pneumococcal cell surface lipoteichoic acid enhances repulsion from the positively charged NETs [107].

Besides their bacterial killing functions, neutrophils augment inflammatory reactions causing bystander lung injury. The net outcome of the neutrophil influx during pneumococcal pneumonia can be beneficial or deleterious, based on the virulence and serotype of the pneumococci [108].

\section{Adaptive Immunity}

Adaptive immune effectors are crucial for developing protective immunity during pneumococcal infections and conjugated polysaccharide capsule vaccination. B cells and subsets of T cells mediate protective adaptive defense against pneumococci.

Discovery of antibody-mediated immunity against pneumococci was based on very early studies in the 19th century on the protective functions of the serum isolated from rabbits that were infected or immunized against pneumococcal infections [68] Systemic antibodies specific against the pneumococcal polysaccharide capsule are the most critical mediator for humoral protective immunity against invasive pneumococcal infections. Differences in capsule structure led to production of antibodies with different specificities which were the basis for serotyping and identification of more than 90 serotypes of pneumococci so far. Moreover, several pneumococcal surface proteins, lying beneath or interspersed within the polysaccharide capsule, such as PspA and PsaA, are immunogenic, inducing the production of pneumococcal-specific antibodies. These antigens are conserved among most pneumococcal serotypes; thus their specific 
antibodies can provide broad protection. However, vaccines composed of individual pneumococcal surface antigens are less potent than capsular polysaccharide vaccines, but the potency can be synergized if they are combined [109].

There are two mechanisms for activating naïve B cells to secrete pneumococcalspecific antibodies: thymus-dependent and thymus-independent. Polysaccharide capsular antigens stimulate terminally differentiated B cell proliferation by crosslinking specific B cell receptors followed by secretion of specific antibodies and limited isotype switching with the help of activating cytokines. It is considered a thymus-independent manner due to the inability of capsular polysaccharides, except zwitterionic polysaccharides, to be processed and presented on MHC class II for specific $\mathrm{CD}^{+} \mathrm{T}$ cell activation. In humans, the isotypes of capsular-specific antibodies are restricted to $\operatorname{IgM}$ and $\operatorname{IgG} 2$, and to a lesser extent IgG1, while they are IgM and IgG3 isotypes in mice [110]. This mechanism induces a rapid increase in antibody titers, but it is transient due to the inability to generate memory B cells. Therefore, repeated exposure to the capsule does not boost specific antibody titers. Although they are elicited in a $\mathrm{T}$ cell-independent manner, some studies showed a significant influence of $\mathrm{CD}^{+} \mathrm{T}$ cells on IgG responses to pneumococcal capsular polysaccharides [111].

In contrast, pneumococcal surface proteins and other protein antigens can be processed and presented for specific $\mathrm{CD} 4{ }^{+}$Th cell activation and consequently elicit thymus-dependent activation of specific B cells. Thus, B cells undergo clonal expansion and differentiation into specific antibody-secreting plasma cells and memory B cells. Specific antibody titers increase slowly but last longer and can be boosted by repeated antigen exposures due to the presence of specific memory B cells. Likewise, the proteinconjugated pneumococcal capsular polysaccharide vaccines stimulate Th-dependent B cell responses, as the co-expression of proteins with the capsular polysaccharides stimulates $\mathrm{CD} 4^{+}$Th cells which activate capsular polysaccharide-specific B cell responses associated with specific memory development, leading to a superior potency over the pure polysaccharide vaccines $[109,110]$.

The protective functions of pneumococcal-specific antibodies are mainly implemented through binding to the pneumococcal surface, which enhances opsonophagocytosis and clearance of pneumococci through Fc receptors on phagocytes or by classical activation of the complement system. The effectiveness of mucosal pneumococcal-specific IgA is limited due to bacterial expression of a secreted zinc metalloprotease, IgA1 protease that specifically targets human immunoglobulin A1 (IgA1), which constitutes more than $90 \%$ of the IgA in the human airway [76].

Additionally, antibody-independent $\mathrm{CD}^{+} \mathrm{T}$ cell-mediated immunity has been shown to mediate protection against pneumococcal infections [112]. The importance of $\mathrm{CD} 4{ }^{+} \mathrm{T}$ cells in the protective immunity is clearly evident in HIV patients, as HIV infection results in a 50 -fold increased risk of pneumococcal infections that is inversely related to $\mathrm{CD}^{+}{ }^{+} \mathrm{T}$ cell count [113]. In animal studies using intranasal immunization with killed pneumococcal whole cell vaccine, pneumococcal-specific CD4 ${ }^{+}$Th17 cells display a significant role in the protection against pneumococcal colonization, which is mediated 
by IL-17A. Their protective role involves inducing recruitment of phagocytes, including macrophages and neutrophils, which accelerate pneumococcal clearance $[109,114,115]$.

The role of $\mathrm{CD} 8^{+} \mathrm{T}$ cells during pneumococcal infections and vaccination is controversial. Animal studies using pneumococcus serotype 3 showed an essential role for $\mathrm{CD} 8^{+} \mathrm{T}$ cells in antibody-mediated pulmonary protection, but not systemic immunity, against pneumococcal infections [116, 117]. In contrast, another study suggested suppressive activity of $\mathrm{CD} 8^{+} \mathrm{T}$ cells against capsular polysaccharide-specific antibody responses in vaccinated mice [118]. Skewed Th17 cell responses were observed during pulmonary pneumococcal infections in mice with genetic deletion of $\mathrm{CD} 8^{+} \mathrm{T}$ cells, which may have deleterious inflammatory outcomes [117]. Therefore, the role of $\mathrm{CD}^{+} \mathrm{T}$ cell responses may be partly justified through their regulatory influence on Th17 cell-induced inflammatory activities.

\section{Respiratory Bacterial Super-infections}

\section{Significance}

Influenza and pneumonia caused the largest number of infectious disease-related deaths in the United States throughout the 20th century [119]. Respiratory infections still cause major public health problems with enormous clinical and socioeconomic burdens. The rates of respiratory infections are higher than any other infectious disease worldwide [120]. Generally, influenza infections are mild, and most influenza-associated morbidity and mortality are not caused by the viral-induced damage itself. Instead, bacterial superinfections have been among the major reasons for the increased mortality during influenza epidemics and pandemics [35].

René Laennec, who invented the stethoscope, was the first physician to describe the "double" pneumonia as a common fatal complication during an 1803 influenza epidemic in France. Throughout the recorded influenza epidemics and pandemics in the modern history, secondary bacterial pneumonia (SBP) was a major complication in severe and fatally-infected cases [121]. For example, the 1918 H1N1 influenza pandemic was devastating, as it killed more than 30 million people worldwide, which is more than three times the number of people killed during World War I [122]. Recently, it was shown that more than $90 \%$ of autopsy lung tissues of the dead people were positive for SBP, which was predominantly caused by $S$. pneumoniae [123]. Furthermore, the lethal effects of SBP were also evident during later influenza pandemics in the antibiotic era, as more than $50 \%$ of the fatal cases during the $1957 \mathrm{H} 2 \mathrm{~N} 2$ and the $1968 \mathrm{H} 3 \mathrm{~N} 2$ influenza pandemics had SBP but with a significant remarkable contribution of Staphylococcus aureus [123-125]. In addition, during the $2009 \mathrm{H} 1 \mathrm{~N} 1$ influenza pandemic, about $25-50 \%$ of fatal or severe cases had SBP $[35,36,126]$. In addition, more than $50 \%$ of children infected with either pandemic $2009 \mathrm{H} 1 \mathrm{~N} 1$ influenza or seasonal influenza had bacterial co-infection, mainly by Staphylococcus aureus, and S. pneumoniae [127]. 
Certain bacterial pathogens have been commonly isolated from patients with secondary bacterial infections, such as S. pneumoniae, Staphylococcus aureus, and Haemophilus influenzae [123, 128, 129]. Recent analysis of pooled human cases with bacterial co-infections from 1950 to 2006 showed that pneumococcus was the most common cause of bacterial co-infection with influenza. It accounted for about $40 \%$ and $16 \%$ of bacterial co-infections during pandemic and seasonal periods, respectively [128].

Given the significant frequency and effect of bacterial super-infections after influenza, it is of paramount importance to study this respiratory viral-bacterial synergism. Exploring key factors mediating this synergism will facilitate investigating effective preventive and therapeutic approaches during seasonal and epidemic influenza periods. In addition, it will allow better strategic preparedness for any future influenza pandemics.

\section{Mechanisms of Respiratory Viral-bacterial Synergism}

Respiratory viral-bacterial synergism is a complex multifactorial phenomenon. It involves several viral, bacterial, and host factors contributing to increased susceptibility and the pathogenesis of bacterial super-infections following several types of respiratory viral infections including influenza. However, the classical dogma explaining this synergism has relied on the viral-induced damage in the respiratory epithelium exposing more niches for bacterial adherence $[35,130]$. This concept would be a major factor in viral-bacterial synergism if the synergism was only observed with highly virulent viruses. Yet, low pathogenic viruses have also been found to enhance susceptibility to bacterial super-infections, as seen with seasonal influenza viruses [131, 132].

Four main mechanisms have been proposed to explain this synergism. These mechanisms essentially describe factors underlying how primary viral infections establish niches for secondary bacterial invaders and support their spread, virulence, and outgrowth, while impairing host antibacterial defense lines (Figure 1-6).

The first category involves factors establishing foothold for secondary bacterial pathogens which is the first step in successful bacterial infection. The breakdown of respiratory epithelial barrier integrity, due to the death of influenza-infected cells lining the airways, exposes extracellular matrix proteins providing more sites for bacterial attachment. In addition, influenza NA activity cleaves sialic acid residues on the host mucosal surface exposing more receptors for bacterial adherence. Increased viral NA activity was shown to support SBP development [133]. This may also be explained by facilitating viral spread deep in the lungs. Strikingly, released sialic acid residues, a good carbon and energy source, are involved in several activities that support pneumococcal biofilm formation in vitro and invasiveness in the lungs $[35,134]$. Furthermore, plateletactivating factor receptor expression is upregulated during influenza infection which may enhance pneumococcal invasiveness into the blood. However, it was shown to be inessential for SBP progression [35, 135]. 


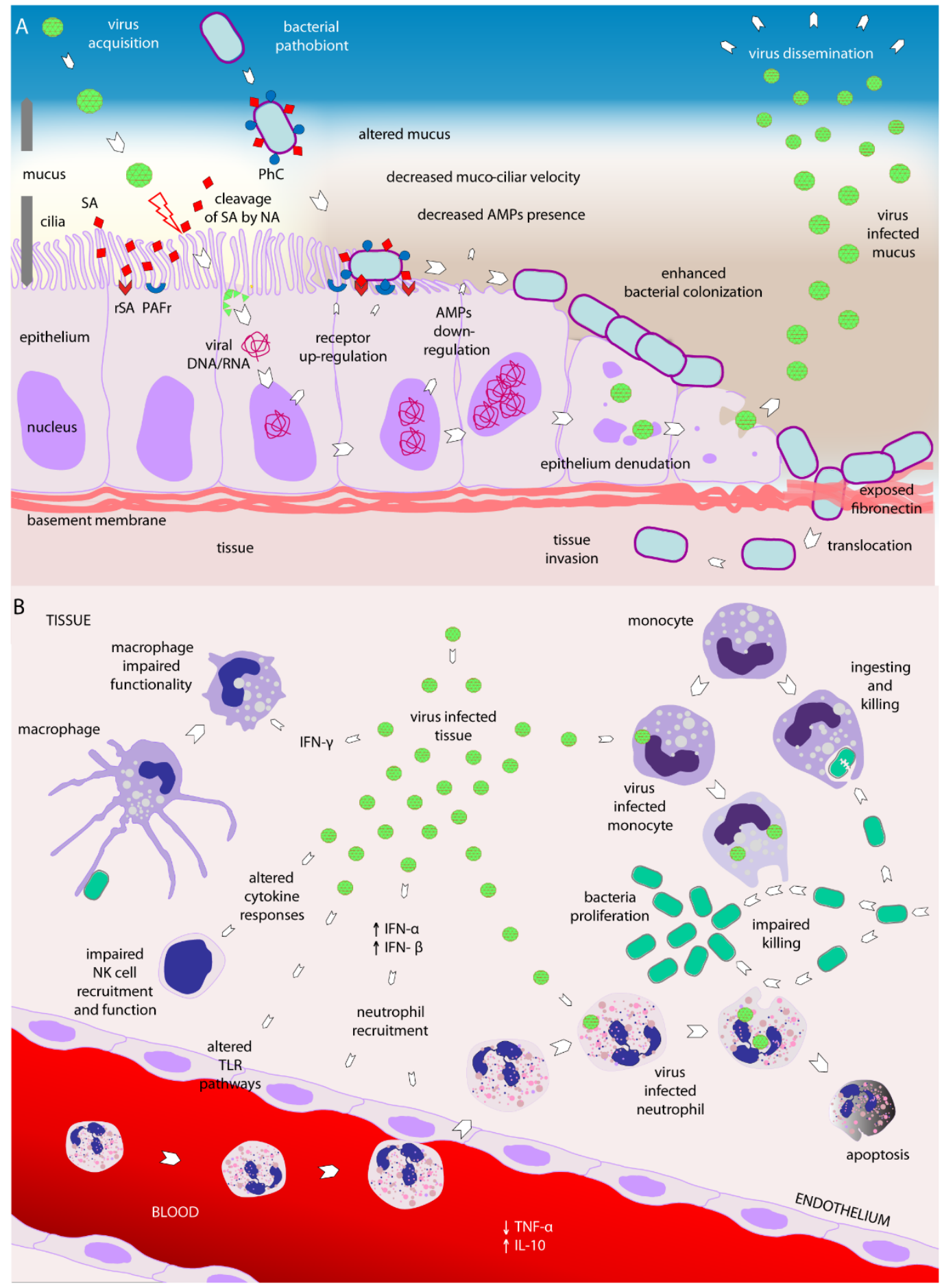

\section{Figure 1-6. Respiratory viral-bacterial interactions}

Reprinted with permission from PLOS. Bosch AA, Biesbroek G, Trzcinski K, Sanders EA, Bogaert D. Viral and bacterial interactions in the upper respiratory tract. PLoS pathogens 2013; 9:e1003057 [136]. 
The second category involves factors facilitating bacterial spread deep in the lungs. Several respiratory viral infections, such as influenza and respiratory syncytial virus infection, inhibit mucociliary beating. Thereby, they impair early mechanical clearance of invading bacteria from the trachea, which promotes deep bacterial spread within the lungs $[137,138]$. Additionally, viruses with higher tropism to replicate deep within the lungs, such as the 2009 pandemic H1N1 influenza virus, may establish niches for secondary bacterial pathogens causing lower respiratory tract infections and SBP [35].

The third category proposes bidirectional synergistic interactions between influenza viruses and the secondary bacterial pathogens, rather than the previous mechanisms that involve unidirectional effects through the influenza viral pathogenicity in the lungs. Indeed, specific pairings of viral and bacterial pathogens show successful synergism in the lungs. In addition, different bacterial strains have remarkable differences in their disease potential after primary influenza viral infection [139]. Bacterial virulence factors may augment the pathogenesis of primary influenza virus infections. However, only few of these factors are known [139]. For instance, proteases from Staphylococcus aureus showed a synergistic effect on the infectivity of influenza viruses by activating HA by cleavage, which increased co-infection pathogenicity [140]. Other examples of bidirectional interactions include synergistic inflammatory responses during secondary bacterial infections. Pathogenesis of primary influenza infections is believed to be mainly due to inflammatory cytokine responses and host immunopathology. During bacterial coinfections, the host immunopathology is augmented at the site of infection. However, this interaction is usually observed in a synergistic rather than an additive fashion, which might be due to the remarkable increase in expression of certain PRRs in the lungs and some circulating immune cells during influenza infections, such as TLR2, TLR3, and TLR9 $[141,142]$. Additionally, during the acute phase of the primary influenza infection, the accumulated inflammatory immune cells would add to the total numbers of the detecting system in the lungs. Thus, in addition to ongoing inflammatory responses during influenza infection, secondary bacterial invaders elicit synergistic inflammatory reactions after activating more PRRs and more immune cells. However, genetic deletions of some TLRs, such as TLR2, did not prevent SBP development or lethal bacterial coinfections $[143,144]$.

The last category of the proposed mechanisms underlines the dysfunction of host antibacterial immunity during primary influenza infections which leads to uncontrolled bacterial outgrowth. Pathogenic bacteria invading terminal airways are encountered by the host first line of innate defense composed of specialized highly phagocytic cells within the alveolar airspaces - the resident AMs. Several studies of animal models reported phagocytic dysfunction in AMs during influenza infections [145-148]. A more recent study attributed this dysfunction to IFN $\gamma$-mediated downregulation of the expression of a certain scavenger receptor, MARCO receptor, on the surface of AMs. Thereby, their phagocytic capacity was decreased, and early pneumococcal clearance was impaired during the resolution phase of sublethal influenza infection when adaptive immune cells infiltrate the lungs with increased IFN $\gamma$ production [101]. In addition, in vitro incubation of murine macrophages with IFN $\gamma$ may decrease macrophage expression 
levels of another scavenger receptor - the mannose receptor, and phagocytic functions against certain bacterial pathogens $[149,150]$.

Viral PB1-F2 protein has also been implicated in viral-bacterial synergism and its associated lung immunopathology. This viral protein is the product of an alternate open reading frame of the PB1 viral gene segment in some influenza virus strains, with variable expression in infected host cells leading to strain-specific pathogenicity [9]. It has multifunctional properties, mainly contributed to by its pro-apoptotic and proinflammatory motifs [151]. Its expression leads to an enhanced inflammatory response and lung immunopathology, contributing to the increased mortality in murine models of SBP [152-154]. Furthermore, it has been suggested that it may induce the death of AMs, thereby contributing to the impairment of antibacterial innate immunity during influenza infections [155].

Impaired functions of murine NK cells during influenza infections have also been implicated to contribute to the enhanced susceptibility to secondary Staphylococcal infections, probably by decreasing the opsonophagocytic functions of AMs gated as $\mathrm{CD} 11 \mathrm{~b}^{\text {hi }} \mathrm{Gr} 1^{\text {low }} \mathrm{CD} 11 \mathrm{c}^{\text {low }}$ cells [156]. However, the role of NK cells in the pulmonary antibacterial immunity is still controversial and needs to be fully examined with a variety of bacterial pathogens $[157,158]$.

Neutrophils are considered the second line of innate immune cells, as they are recruited to the lungs when resident AM phagocytic functions are impaired or overwhelmed. Influenza A viruses may accelerate human neutrophil apoptosis in vitro [159]. In addition, neutrophil dysfunction has been proposed to contribute to increased bacterial super-infections after influenza infection [160]. However, the researchers found that pneumococcal titers increased only with co-infection 6 days after influenza infection, although neutrophil dysfunction was seen 3 and 6 days after influenza infection. In addition, they depleted neutrophils using anti-GR1 RB6-8C5 monoclonal antibody, which is a non-selective antibody that could also deplete monocytic cells expressing Ly6C marker, thereby confounding the results [160]. Furthermore, they used a relatively high dose of pneumococcus in their co-infection model, thus overwhelming AM phagocytic capacity. Thus, they ended up examining neutrophil functions. Taken together, the exact impact of influenza infections on neutrophil functions and whether this effect really contributes to increased vulnerability to bacterial super-infections still need to be carefully investigated.

Late after recovery from influenza infection, another mechanism of immune dysfunction has been suggested by the findings of TLR desensitization in the lungs. This mechanism relies on the enhancement of bacterial super-infections by impairing efficient detection of bacterial pathogens by AMs. Thus, it leads to ineffective innate immune responses against secondary bacterial infections [100]. However, this desensitization lasts for several months, which is not clinically relevant to the timing window for successful secondary bacterial infections observed in the first 2 weeks after influenza infection. 
In addition, the early upregulation of the type I IFN response during influenza infection or the increased IL-10 production after the recovery of influenza infection has been suggested to inhibit neutrophil influx which may contribute to the impaired antibacterial innate defense. However, disrupting their signaling pathways provided only partial or minimal resistance against secondary bacterial infections, which was linked to increased neutrophil recruitment and/or functions [161, 162]. Furthermore, increased type I IFN expression during influenza infection was suggested to suppress IL-17 secretion from $\gamma \delta$ T cells that may play important role in recruiting neutrophils during pulmonary bacterial infections. Abrogating their signaling by genetic deletion of IFN alpha receptor improved bacterial clearance and protected mice against SBP following influenza infections $[147,163]$.

Efficient repair of influenza-induced epithelial damage is critical to restoring lung homeostasis and functions. However, during bacterial co-infections, the repair process of damaged epithelial cells is significantly impaired which may contribute to the lethality of bacterial co-infections [144, 164].

In summary, several mechanisms have been proposed with varying levels of evidence supporting their significant contribution to the enhanced susceptibility to secondary bacterial infections following influenza and/or their increased lethality. The current prophylactic and therapeutic measures against this serious medical problem are targeting the infectious agents with either vaccinations or antimicrobial agents, respectively. Indeed, much of these approaches succeeded in decreasing the significance of respiratory secondary bacterial infections compared to the 1918 influenza pandemic time or before. Nonetheless, in the influenza pandemic in 2009-2010, secondary bacterial infections complicated more than one third of severe and fatal cases, even with comprehensive diagnosis and treatment strategies. Many factors may have been involved in this failure, among them is the reported increased emergence of resistant strains of influenza viruses and secondary bacterial pathogens [165-167]. Furthermore, antiviral therapy can only display effective prevention of SBP if administered early during primary influenza infection by shortening the duration of viral shedding $[35,168]$. Therefore, investigating the effect of primary influenza infections on the host pulmonary antibacterial immunity has become of paramount importance, as novel prophylactic or therapeutic approaches based on immunomodulation would be more effective in breaking this synergism by augmenting the host immunity against many secondary bacterial pathogens simultaneously.

\section{Lung-resident Macrophages}

Lungs are part of the respiratory system with unique structure and very critical functions for the human body through blood re-oxygenation at the terminal regions of the lungs - the alveoli. With a very large surface area of about $70 \mathrm{~m}^{2}$ in adult humans and continuous exposure to the external environment with a thin epithelial layer away from the pulmonary capillaries, the alveoli look like a short step away from disaster [169]. Therefore, a complex immune regulatory network functions to maintain the lungs in a 
quiescent non-inflamed state. Critical players in this network are the lung-resident macrophages.

There are two major subsets of lung-resident macrophages: AMs residing in the alveolar lumen within the surfactant thin layer over the alveolar epithelial surface, and interstitial macrophages (IMs) residing in the lung parenchymal tissue. Under steady state conditions, AMs are the major hematopeotic cells in the alveolar airspaces. They were first isolated via pulmonary alveolar lavage technique in 1961 [170]. Since that time, several groups have studied the functions and ontology of pulmonary macrophages which suggested developmental and functional differences between lung-resident AMs and IMs [94, 171, 172]. IMs were suggested as precursors for resident AMs with intermediate maturation stage, while AM ontogeny from circulating blood monocytes or by local macrophage proliferation is still under debate [172-174]. Under inflammatory stimuli, such as influenza infections, different exudate monocytes and macrophages are recruited to the lungs which have different functional phenotypes and probably transcriptional profiles from the lung-resident macrophages $[53,54]$.

Resident AMs play a pivotal role in the immune surveillance in the lungs. Because of their high phagocytic capacity and strong microbicidal activities, they are considered the first line of innate defense against inhaled particles and bacterial pathogens [94, 171]. In addition, they display immunosuppressive activities and poor antigen presenting capability in vitro and in vivo which may be critical to prevent harmless environmental particulates from triggering detrimental inflammatory reactions in the lungs [171, 175-179]. In contrast, IMs have lower phagocytic capacity but better antigen presenting activities, as they express more surface MHC class II molecules and can effectively induce T cell proliferation [94].

Therefore, investigating the impact of influenza infections on the lung-resident macrophages in vivo is an attractive area, as it may elucidate part of the complex puzzle of respiratory viral-bacterial synergism by explaining how influenza infection alters the cellular innate immune defense in lungs and establishes a niche for the development of SBP. This will also open avenues for novel immunomodulating prophylactic and therapeutic interventions to prevent SBP during both pandemic and seasonal influenza periods.

\section{Scope and Objectives of Dissertation}

\section{Specific Aim 1: To Determine the Impact of Influenza Infection on the Lung- resident Macrophages In Vivo}

Sub-aim 1.1. To characterize and differentiate between subsets of lung-resident macrophages and recruited macrophages using in vivo labeling approach. 
Sub-aim 1.2. To track dynamic changes of labeled lung-resident macrophages during PR8 influenza infection.

Sub-aim 1.3. To determine if loss of resident alveolar macrophages during influenza infection is due to increased cell death process.

\section{Specific Aim 2: To Determine if Depletion of Resident Alveolar Macrophages during Influenza Infection Contributes to Enhanced Susceptibility to Secondary Pneumococcal Pneumonia}

Sub-aim 2.1. To determine if early pneumococcal clearance within alveolar airspaces is impaired during the depletion phase of resident alveolar macrophages after influenza infection.

Sub-aim 2.2. To determine if the impaired early pneumococcal clearance facilitates pneumococcal pneumonia development during the depletion phase of resident alveolar macrophages after influenza infection.

\section{Specific Aim 3: To Test if Expanding the Lung-resident Macrophage Pool Can Protect Influenza-infected Mice against Secondary Pneumococcal Pneumonia}

Sub-aim 3.1. To test if local GM-CSF treatment can replenish the alveolar macrophage pool during influenza infection.

Sub-aim 3.2. To determine if the expanded pool of resident pulmonary macrophages, by local GM-CSF treatment, can restore early pneumococcal clearance and prevent secondary pneumococcal pneumonia in influenza-infected mice.

\section{Specific Aim 4: To Test if Adjunctive Immunomodulator Therapy Can Improve Outcomes during Bactericidal Antibiotic Treatment of Secondary Pneumococcal Pneumonia}

Sub-aim 4.1. To determine influence of the lung pneumococcal burden on the lung immunopathology and mortality outcomes during ampicillin treatment of secondary pneumococcal pneumonia.

Sub-aim 4.2. To test efficacy of corticosteroid therapy before or combined with ampicillin treatment of mice with mild or severe secondary pneumococcal pneumonia. 


\section{CHAPTER 2. IMPACT OF INFLUENZA INFECTION ON THE LUNG- RESIDENT MACROPHAGES IN VIVO ${ }^{1}$}

\section{Introduction}

Influenza and pneumonia are leading causes of morbidity and mortality in both children and adults in the United States [180]. In developing countries, acute lower respiratory infections are the leading cause of death in children younger than 5 years of age [181]. Most influenza-related mortality is not due to the viral infection alone. Instead, secondary bacterial pneumonia complicates many severe cases in influenza-infected hosts [35]. This results in a tremendous economic burden due to increased hospitalizations, medical costs, and indirect costs during both pandemic and inter-pandemic influenza periods [123]. Complicating the picture, treatment of secondary bacterial pneumonia may not be successful even in the antibiotics era. Globally increased rates of antimicrobial resistance among many common respiratory bacterial pathogens and the mechanisms of the drugs themselves can both complicate treatment and cure [165, 166, 182, 183]. The continuous threat of a new influenza pandemic makes it crucial to understand how influenza infection alters the host's local innate immunity to the benefit of establishing secondary bacterial infections.

Lungs are protected against bacterial infections by various components of innate and adaptive immunity [44, 184]. Influenza-infected patients are vulnerable to bacterial super-infections, suggesting defects in some or all of these resistance and clearance mechanisms. Certain bacterial pathogens have been commonly isolated from patients with secondary bacterial infections, such as $S$. pneumoniae [123, 128, 129]. Immunity to $S$. pneumoniae is not completely understood at present in intact or compromised hosts. Among host innate immune players, resident AMs are considered to be the most prominent first line of defense against respiratory pneumococcal infections [93], through their high phagocytic capacity [94-96].

Resident AMs therefore represent an intriguing target for study. However, the strategies for differentiation of resident macrophage subsets in the airways and lung tissues are insufficient to distinguish AMs from lung-resident IMs or inflammatory monocytic cells invading the lung in response to infections. In some studies, AMs have been putatively identified based on their surface immunophenotype as CD11 $\mathrm{c}^{\mathrm{hi}} \mathrm{F} 4 / 80^{\mathrm{hi}}$ cells. Indeed, CD11c and F4/80 surface markers are highly expressed on the AM surface, but some inflammatory macrophages/ dendritic cells can express these markers as well [185-187]. Thus, gating on these two markers only will not differentiate the various types of cells during influenza infection, potentially leading to erroneous conclusions about the absolute numbers of resident macrophages.

${ }^{1}$ Adapted with permission from The American Association of Immunologists, Inc. Ghoneim HE, Thomas PG, McCullers JA. Depletion of Alveolar Macrophages during Influenza Infection Facilitates Bacterial Superinfections. J Immunol 2013; 191:1250-9. 
Using a novel in vivo labeling approach combined with extensive flow cytometric analyses and confocal microscopic examination, we efficiently labeled and characterized different subsets of lung-resident macrophages in lungs of naïve mice. We then investigated the impact of sublethal influenza A virus infections on the two major subsets of lung-resident macrophages - alveolar and interstitial macrophages - using a murine model of sublethal influenza infections using different influenza virus strains.

\section{Materials and Methods}

\section{Influenza Viruses}

We used the St. Jude strain of mouse-adapted influenza virus A/Puerto Rico/8/34 (H1N1), referred to as "PR8", as well as the human clinical isolate of the pandemic influenza virus A/California/04/09 (H1N1) referred to as "pdm H1N1". All viruses were passaged once through Madin-Darby canine kidney (MDCK) cells, stocks were grown by a single passage through eggs, and allantoic fluid was stored at $-80^{\circ} \mathrm{C}$. The viral titers of the stocks were characterized via median tissue culture infective dose (TCID50) assay in MDCK cells.

\section{Mice}

Six- to 8-week old female BALB/c mice (Jackson Laboratory, Bar Harbor, ME) were maintained in a Biosafety Level 2 facility in the Animal Resource Center at St. Jude. Animals were given general anesthesia that consisted of $2.5 \%$ inhaled isoflurane (Baxter Healthcare Corporation, Deerfield, IL) prior to all interventions, and all studies were approved by the Animal Care and Use Committee at St. Jude.

\section{Infectious Model}

Infectious agents were diluted in sterile phosphate buffered saline (PBS) and administered intranasally in a volume of $100 \mu \mathrm{l}(50 \mu \mathrm{l}$ per nostril) to anesthetized mice held in an upright position. In all experiments of influenza infection, PR8 influenza virus was given at a dose of 25 doses infectious for $50 \%$ of tissue culture wells (TCID50) per $100 \mu 1$ per mouse, which caused about $10 \%$ weight loss on day 7 after infection and no mortality when given alone. The virus infectious dose in the pdm H1N1 experiment was 600 TCID50 per mouse, which caused morbidity and weight loss comparable to PR8 infection (data not shown). 


\section{In Vivo Labeling of Lung-resident Macrophages}

One hundred $\mu 1$ of 10 mM PKH26-PCL dye (Sigma-Aldrich, St. Louis, MO) was intranasally administered into anesthetized mice 5 days before influenza infection, as previously described [101].

\section{Flow Cytometric Analysis of Immune Cells in Bronchoalveolar Lavage Fluid, Post- lavage Lungs, and Mediastinal Lymph Nodes}

Following euthanasia by $\mathrm{CO}_{2}$ inhalation, the trachea was exposed and cannulated with a 24-gauge plastic catheter (Becton Dickinson Infusion Therapy Systems, Inc., Sandy, UT). Lungs were lavaged 4 times with $1 \mathrm{ml}$ of cold sterile Hank's buffered salt solution (HBSS) supplemented with $0.1 \mathrm{mM}$ ethylenediaminetetraacetic acid (EDTA).The whole lungs after lavage or mediastinal lymph nodes were harvested and physically homogenized by syringe plunger against a $40-\mu \mathrm{m}$ cell strainer and washed in FACS buffer consisting of HBSS, $0.1 \mathrm{mM}$ EDTA, and 1\% heat-inactivated fetal bovine serum. Cell suspension of bronchoalveolar lavage fluid (BALF), post-lavage lung homogenate, or mediastinal lymph nodes homogenate were centrifuged at $4^{\circ} \mathrm{C}, 350 \times \mathrm{g}$ for $7 \mathrm{~min}$, and the BALF supernatant was stored at $-80^{\circ} \mathrm{C}$. Flow cytometry (LSRII, and LSRII Fortessa, Becton Dickinson, San Jose, CA) was performed on the cell pellets after incubation with $75 \mu$ of 1:200 dilution of Fc block (anti-mouse CD16/CD32, BD Bioscience Inc., San Jose, CA) on ice for $10 \mathrm{~min}$, followed by surface marker staining with anti-mouse antibodies conjugated with different fluorescent probes: CD11c (eFluor 450), F4/80 (FITC), Ly6G (PerCp-Cy5.5), Ly6C (APC), and CD11b (APC-eFluor 780; eBioscience Inc., San Diego, CA). The data were analyzed using FlowJo 8.8.6 (Tree Star, Ashland, OR) where viable cells were gated from an FSC/SSC plot. First, neutrophils $\left(C D 11 b^{\text {hi }}\right.$ Ly6G $\left.{ }^{\text {hi }}\right)$ were gated out, then macrophages (CD11 $c^{\text {hi }}$ F4/80 ${ }^{\text {hi }}$ ) were sub-gated based on CD11b surface expression into AMs (CD11b $)$, IMs (CD11 $\left.b^{\text {low-int }}\right)$, and recruited exudate macrophages $\left(\mathrm{CD} 11 \mathrm{~b}{ }^{\mathrm{hi}}\right)$. Viable and non-viable cells were counted before surface marker staining, and the percentage viability was counted via the trypan blue exclusion method using a Cell Countess System (Invitrogen, Grand Island, NY). The absolute numbers of different cell types were calculated based on the proportion of viable events analyzed by flow cytometry as related to the total number of viable cells per sample. Live/dead aqua dye was added during flow cytometric surface staining to determine the total numbers of dead AMs and IMs. This dye binds to free amines after penetrating the impaired cell membrane of dead cells. First, AMs and IMs were gated as mentioned above but without prior gating for viable events based on forward scatter and side scatter (FSC/SSC) dot plot. Live/dead aqua-positively stained AMs and IMs were gated as dead cells, and their numbers were calculated based on the proportion of dead events from the total events, analyzed by flow cytometry. Average mean fluorescence intensity of PKH26-PCL was measured for different subsets of macrophages in the PE channel. 


\section{Confocal Laser Scanning Microscopy of PKH26-labeled Lungs}

Five days after in vivo PKH26-PCL labeling of lung macrophages, euthanasia by $\mathrm{CO}_{2}$ inhalation was performed, and the trachea was exposed and cannulated with a 24 gauge plastic catheter (Becton Dickinson Infusion Therapy Systems, Inc., Sandy, UT). Lungs were harvested after instillation of $1.2 \mathrm{ml}$ of $4 \%$ freshly prepared formaldehyde with PBS. Harvested lungs were fixed in 4\% formaldehyde with PBS at room temperature for 1 hour. Every lung was then washed with PBS and cut into 4 pieces before mounting into PBS in 4 wells of Nunc Lab-Tek chambered cover glass (Thermo Scientific, Rochester, NY) before microscopic examination. Fluorescence was visualized with a Nikon Eclipse TE2000-E inverted microscope equipped with C2 confocal system and a 40X/1.3NA numeric aperature oil objective (Nikon Instruments, Melville, NY). Image collection and analysis were performed with Nikon NIS-Elements software (Version 4.13).

\section{Cytospin Slides Preparation}

After harvesting BALF as described above, and before staining cells for flow cytometry analysis, BALF cells were resuspended in PBS, cytospun (Thermo Scientific, Ashville, NC) onto glass slides, and stained with Diff-Quick (Quik-Dip stain; Mercedes Medical, Sarasota, FL). Neutrophils, monocytes/macrophages, and lymphocytes were identified by morphology and images were taken from different representative fields of stained macrophages under high power fields.

\section{Statistical Analysis}

Comparison of cell counts, and mean fluorescence intensity of PKH26-PCL in BALF, and post-lavage lung homogenate between groups were compared using analysis of variance (ANOVA). Mann-Whitney U test was used for pair-wise comparisons of mean fluorescence intensity of PKH26-PCL between AMs and IMs in fixed lungs under confocal microscopy, or comparisons of Ly6C surface expression between AMs, and IMs, or recruited macrophages. A p-value of $<0.05$ was considered significant for these comparisons. Prism 4 for Windows (GraphPad Software, Inc., V 4.03) was used for all statistical analyses.

\section{Results}

\section{In Vivo Labeling of Lung-resident Macrophages Can Distinguish Alveolar Macrophages from Interstitial Macrophages}

Before examining any alterations in the lung-resident innate immune cells in influenza-infected hosts, we determined critical techniques for the differential analysis of 
the heterogeneous population of macrophages in both alveolar airspaces and post-lavage lung tissue. First, we refined a flow cytometry technique for gating resident macrophages so that clear differentiation between different cells types could be accomplished. We used the common markers CD1 $1 \mathrm{c}^{\mathrm{hi}}$ and F4/80 ${ }^{\mathrm{hi}}$ and added gating by another marker, CD11b ( $\beta 2$-integrin). CD11b has been shown to be weakly expressed on AM cell surface [172, $188,189]$ while highly expressed on granulocytes, exudate macrophages, monocytes, and some dendritic cells [190-193]. Therefore, AMs are better gated as CD1 $1 \mathrm{c}^{\text {hi }} \mathrm{F} 4 / 80^{\text {hi }}$ CD11 b ${ }^{\text {dim }}$ in BALF as shown recently $[189,194]$. This allowed differentiation in our flow cytometric analysis of the two major subsets of lung-resident macrophages in mockinfected mice. Thus, AMs could be gated as CD11 $\mathrm{c}^{\mathrm{hi}} \mathrm{F} 4 / 80^{\mathrm{hi}} \mathrm{CD} 11 \mathrm{~b}^{-}$and IMs as $\mathrm{CD} 11 \mathrm{c}^{\mathrm{hi}}$ $\mathrm{F} 4 / 80^{\text {hi }} \mathrm{CD} 11 \mathrm{~b}^{\text {low-int }}$ in both BALF and post-lavage lung homogenate (Figure 2-1A).

To confirm our gating strategy for both subsets of lung-resident macrophages and to differentiate them from recruited macrophages, we did in vivo labeling of lungresident macrophages before influenza infection using intranasally administered PKH26-Phagocytic Cell Labeling (PKH26-PCL) dye. Both subsets of lung-resident macrophages were intensely labeled by the dye, showing high means of the fluorescence intensity of PKH26-PCL dye (MFI-PKH26). Nonetheless, significant differences in MFIPKH26 were observed between AMs and IMs in both BALF and post-lavage lung homogenates, with IMs showing significantly lower MFI-PKH26 than AMs (Figure 2-1B). Additionally, selective labeling of lung-resident macrophages was confirmed by confocal microscopy of fixed naïve PKH26-labeled lungs showing the same pattern of difference in PKH26-MFI (Figure 2-2). These differences reflect the spatial and functional differences between the two major subsets of lung-resident macrophages, with IMs having less accessibility to the intranasally administered dye and lower phagocytic capacity than AMs $[94,172,195]$. Thus, the in vivo labeling method confirmed the validity of our flow cytometric gating strategy for the lung-resident macrophage subsets.

\section{Influenza Virus Infection Depletes Alveolar Macrophages}

To determine if influenza infection causes any alteration in the numbers of lungresident macrophages, we determined the percentages of lung-resident macrophages in flow cytometric dot plots and calculated their absolute numbers. We infected BALB/c female mice intranasally with the mouse-adapted $\mathrm{H} 1 \mathrm{~N} 1$ influenza virus strain $\mathrm{A} /$ Puerto Rico8/34 (PR8) using a sublethal dose (25 TCID50 per $100 \mu \mathrm{l})$. Seven days after influenza virus infection, more than $90 \%$ of the AM pool was depleted (Figure 2-3A). To exclude the possibility that the observed depletion of AMs is unique to the use of a mouse-adapted influenza virus strain (PR8), we examined changes in the AM pool size using a sublethal dose of a human clinical influenza isolate from the 2009 influenza pandemic, the A/California/04/09 H1N1 strain (pdm H1N1). We found that AMs were significantly depleted within 7 days after pdm H1N1 infection to levels comparable to PR8 infection (Figure 2-3B). Furthermore, we also tested the impact of sublethal influenza B virus infection in vivo, and we found significant depletion of resident AMs on days 3 and 5 after infection (data not shown). 

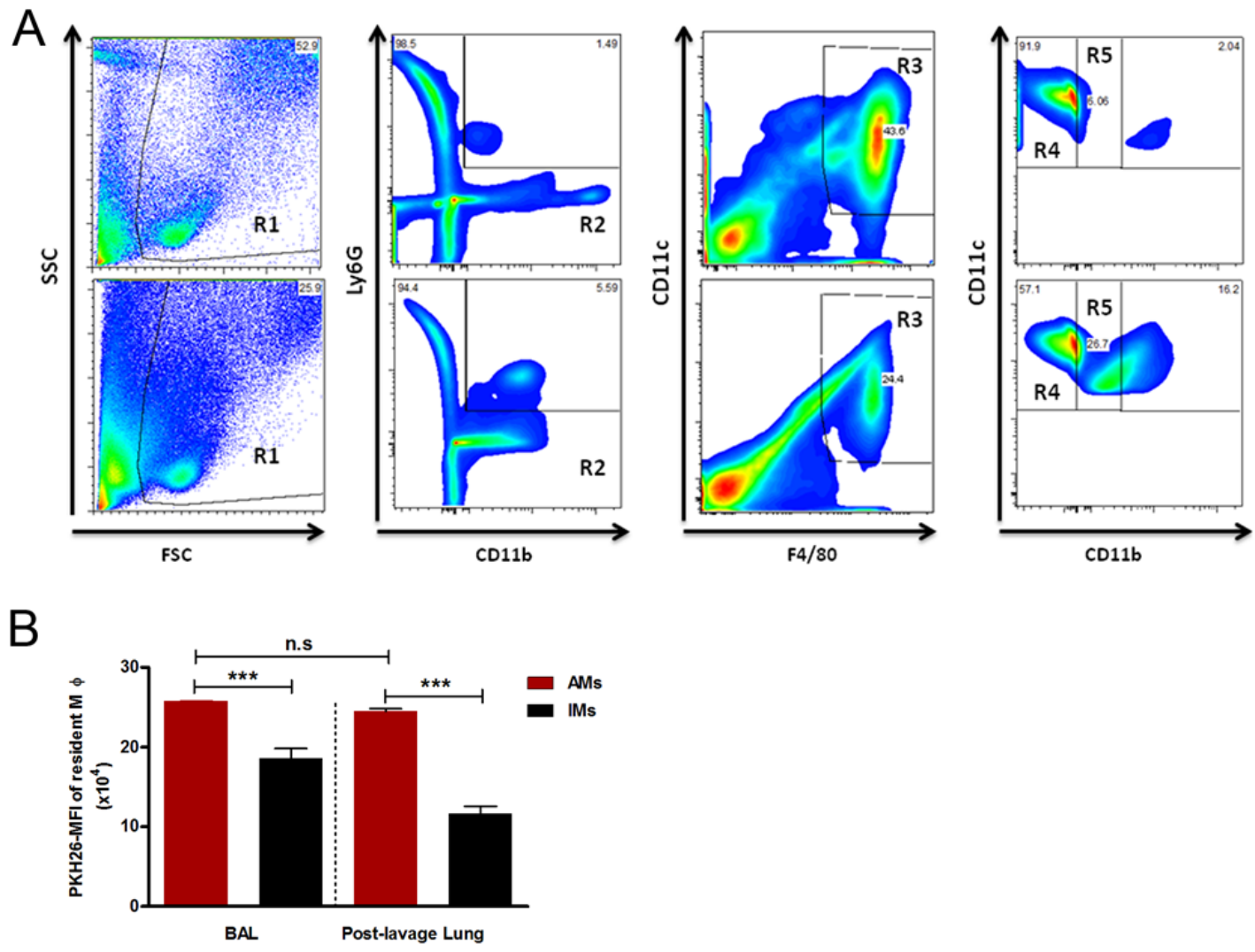

Figure 2-1. Differentiation between lung-resident macrophages subsets

(A) Flow cytometry dot plots show the gating strategy of resident alveolar macrophages (AMs, R4 gate) and interstitial macrophages (IMs, R5 gate) in BALF (top plots) and post-lavage lungs (bottom plots) of mock-infected mice. (B) In vivo labeling of lungresident macrophages using $\mathrm{PKH} 26-\mathrm{PCL}$ dye before influenza infection can distinguish AMs (solid red bars) from IMs (solid black bars) based on the mean fluorescence intensity (MFI) of PKH26-PCL dye in both BALF and post-lavage lungs $(n=4)$. $* * * P<0.001$ by Tukey's multiple comparison test (ANOVA). The bar graphs show the average $\pm \mathrm{SD}$. 

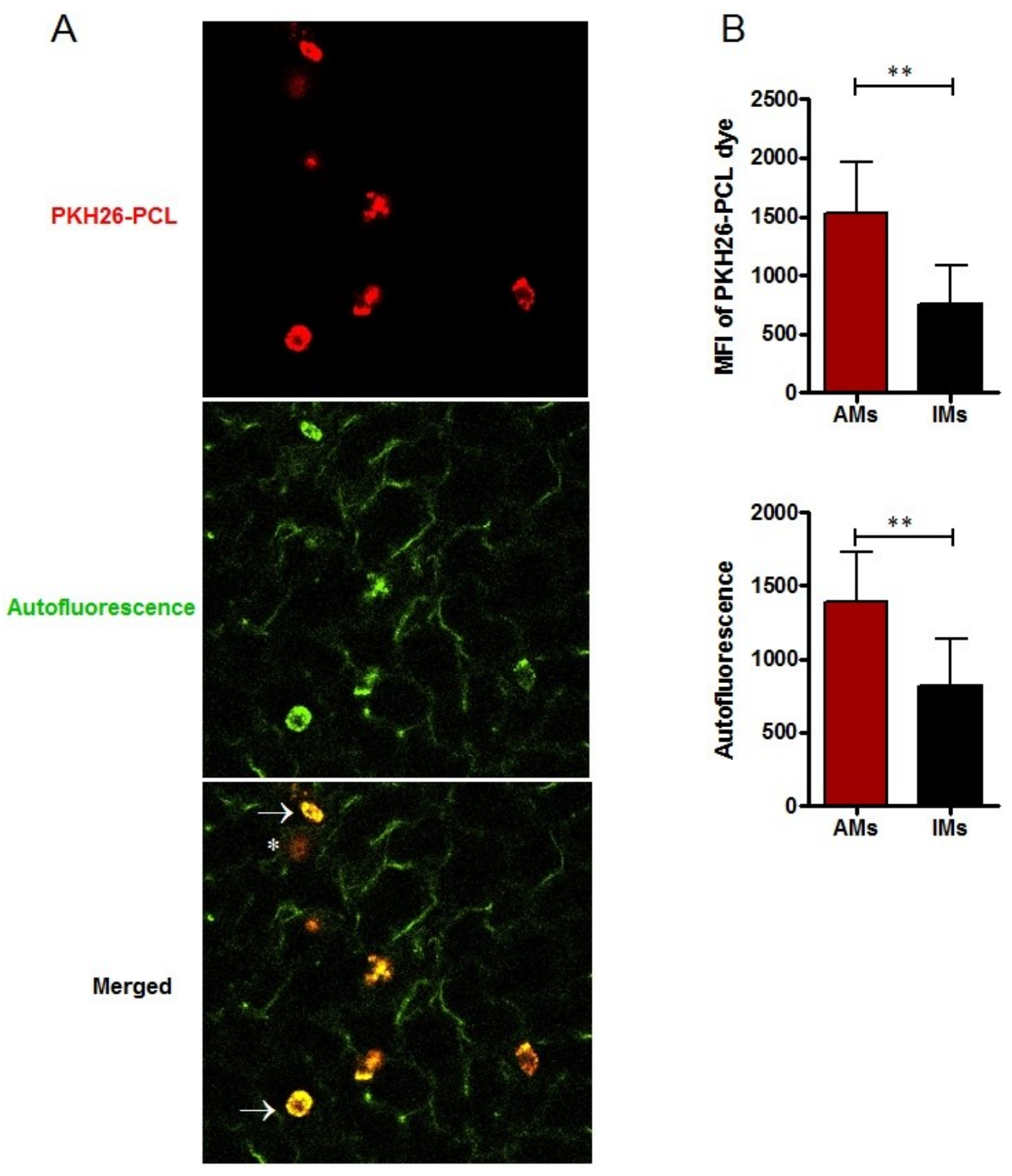

Figure 2-2. Fluorescence microscopic examination of PKH26-labeled naive lungs confirms selective in vivo labeling of lung-resident macrophages

(A) Photomicrographs show that both AMs (arrow) and IMs (asterisk) are selectively labeled by PKH26-PCL dye and emit red PKH26 fluorescence. Green autofuorescence can be detected from AMs, IMs, and elastin and collagen fibers. (B) AMs have significantly higher PKH26-MFI and autofluorescence compared with that of IMs measured from 3 different fields. Images were obtained by a Nikon Eclipse TE2000-E microscope $(\mathrm{n}=3)$.

$* * P<0.01$ by Mann-Whitney $\mathrm{U}$ test. The bar graphs show the average $\pm \mathrm{SD}$. 
A

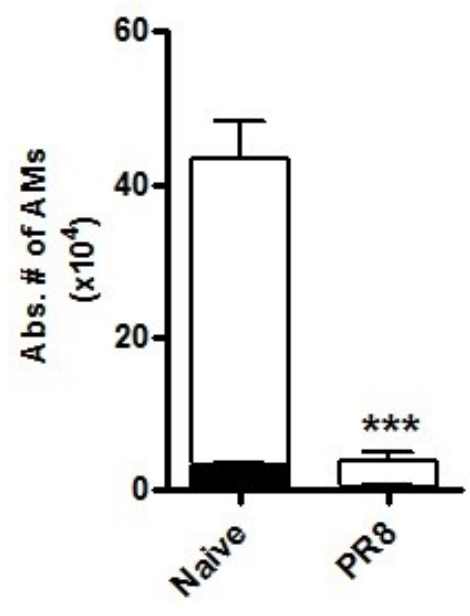

B

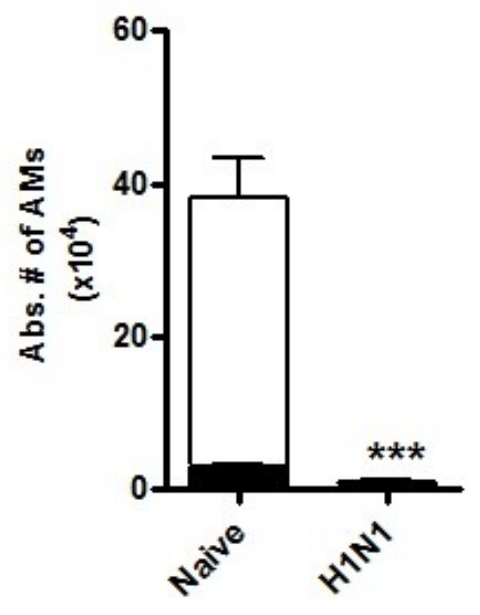

Figure 2-3. Lung-resident alveolar macrophages are depleted during influenza virus infections

Absolute numbers of AMs in bronchoalveolar lavage fluid (solid bars) and post-lavage lungs (open bars) of PR8-infected (A), or 2009 pandemic H1N1-infected mice (B), analyzed 7 days after influenza infection, are significantly lower than in mock-infected (naive) mice ( $\mathrm{n} \geq 5$, in each group of mock-infected or influenza-infected mice).

$* * * P<0.001$ by Mann-Whitney U test, compared with mock-infected (naïve) mice. The bar graphs show the average \pm SEM. 


\section{Tracking of Lung-resident Macrophage Dynamic Changes during Influenza Infection}

To track depletion kinetics of AMs and changes of the IM pool size during influenza infection, we again did in vivo labeling of lung-resident macrophages followed by sublethal PR8 influenza infection and studied different time points in the first 2 weeks after influenza virus infection. We found that AMs were significantly depleted in the alveolar airspaces shortly after PR8 infection starting on the first day p.i. (Figure 2-4A). Considering that some AMs may not be completely harvested in BALF and still adhere to the respiratory tract lining, we also analyzed post-lavage lungs and found that higher numbers of AMs remained in the post-lavage lung homogenate. These remaining AMs were significantly depleted starting 3 days p.i. compared with those in mock-infected mice (Figure 2-4B). Consequently, 3 days after PR8 influenza infection was identified as the earliest time point of significant whole lung AM depletion. The AM pool was partially replenished 9 days p.i. (in BALF only), while complete replenishment appeared 11 days p.i. (Figure 2-4A and B). Conversely, the IM pool was not significantly depleted during the course of influenza infection; however, it was expanded at later time points (Figure 2-4A and B).

By tracking dynamic changes in MFI-PKH26 of AMs and IMs after PR8 influenza infection, we observed that MFI-PKH26 of AMs significantly decreased during the full replenishment period starting 11 days p.i (Figure 2-5A and B). In contrast, MFIPKH26 of IMs decreased shortly after influenza infection without significant changes in their absolute numbers observed in the post-lavage lungs in the first week of infection (Figure 2-4B and Figure 2-5B). However, in the second week, the IM pool significantly expanded with a significant increase in their absolute numbers harvested from BALF (Figure 2-4A). This may suggest partial depletion of IMs pool which can be quickly restored through their rapid proliferative capacity.

\section{Validity and Stability of the In Vivo Labeling Approach to Distinguish Different Subsets of Pulmonary Macrophages during the Tracking Period}

To confirm the validity of the tracking process of lung-resident macrophages during the course of influenza infection, dynamic changes in their MFI-PKH26 were measured. At any time point after influenza infection, AMs showed the highest MFIPKH26 compared to IMs or recruited exudates macrophages (Figure 2-6A and B), despite the observed decrease in MFI-PKH26 of AMs during their full replenishment period starting 11 days p.i. (Figure 2-5A and B). Thus, it confirms our flow cytometric gating strategy that differentiates between resident AMs and IMs, and newly recruited exudate macrophages.

We did further characterization of the different macrophage subsets in the lungs during influenza infection based on their surface expression levels of Ly6C antigen that can be used to differentiate between resident and recruited inflammatory macrophage subsets, in addition to their maturation stages [191, 192]. We found that resident AMs did 

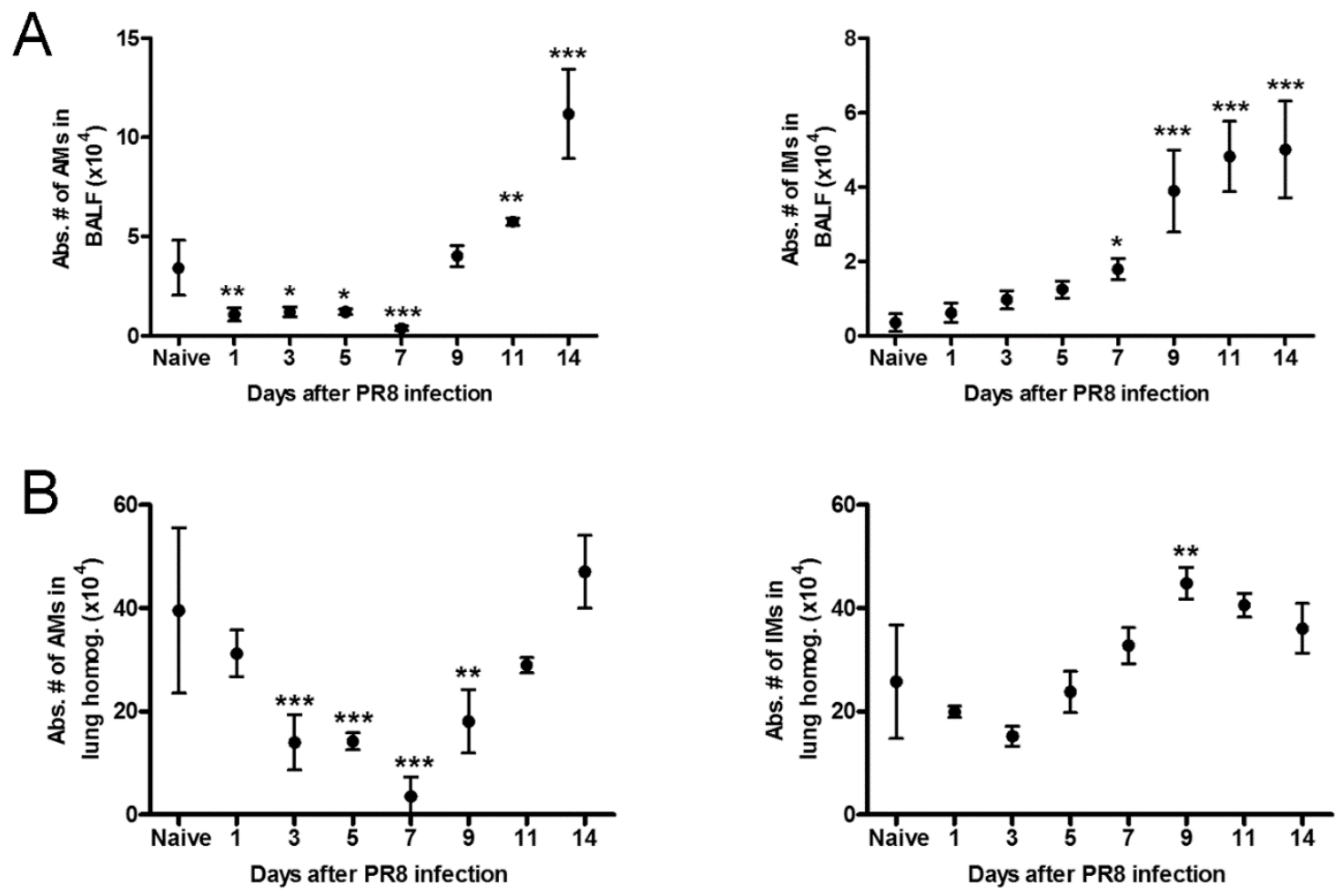

Figure 2-4. Tracking of lung-resident macrophage dynamic changes during influenza infection

Absolute numbers of resident AMs and IMs in BALF (A) and post-lavage lungs (B) of PR8-infected mice harvested $1,3,5,7,9,11$, and 14 days p.i $(\mathrm{n} \geq 4)$.

${ }^{*} P<0.05,{ }^{*} P<0.01,{ }^{*} * P<0.001$ by Dunnett's multiple comparison test (ANOVA), compared with mock-infected (naïve) mice. Data are expressed as the average \pm SD. 

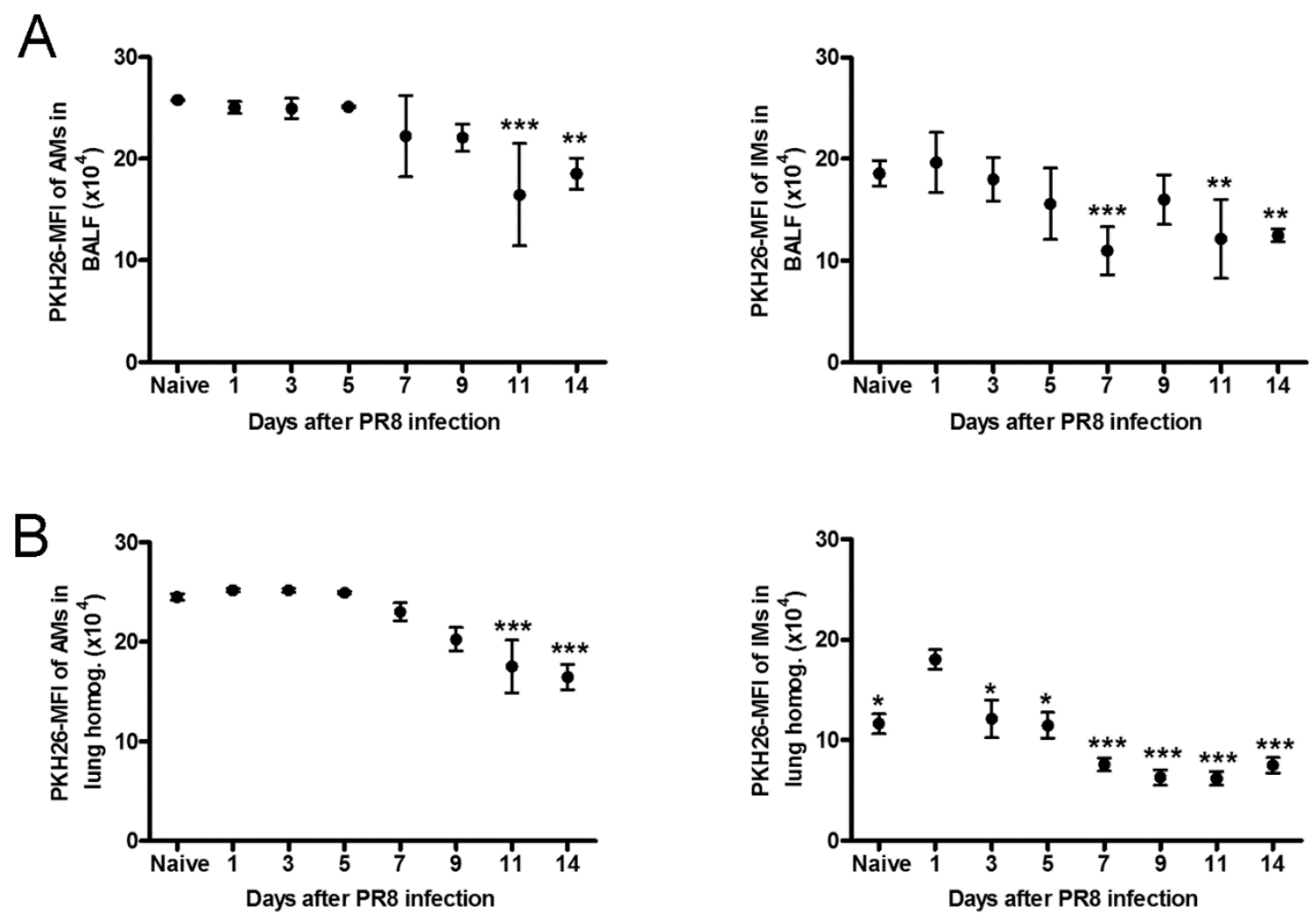

Figure 2-5. Dynamic changes of the mean fluorescence intensity of PKH26-PCL of the lung-resident macrophages during influenza infection

Tracking the changes in MFI (mean fluorescence intensity) of PKH26-PCL dye of resident AMs and IMs in BALF (A) and post-lavage lungs (B) at different time points during influenza infection $(\mathrm{n} \geq 4)$.

${ }^{*} P<0.05, * * P<0.01,{ }^{*} * P<0.001$ by Dunnett's multiple comparison test (ANOVA), compared with influenza-infected mice 1 day p.i. Data are expressed as the average \pm SD. 


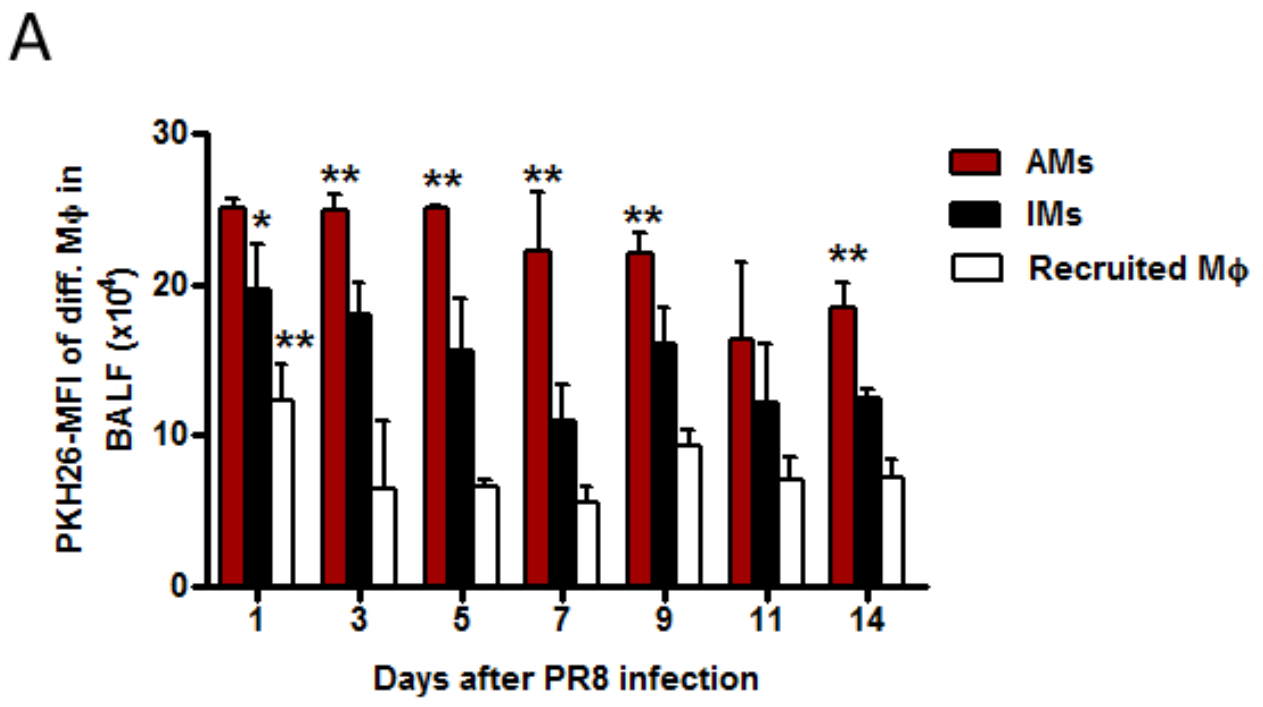

B

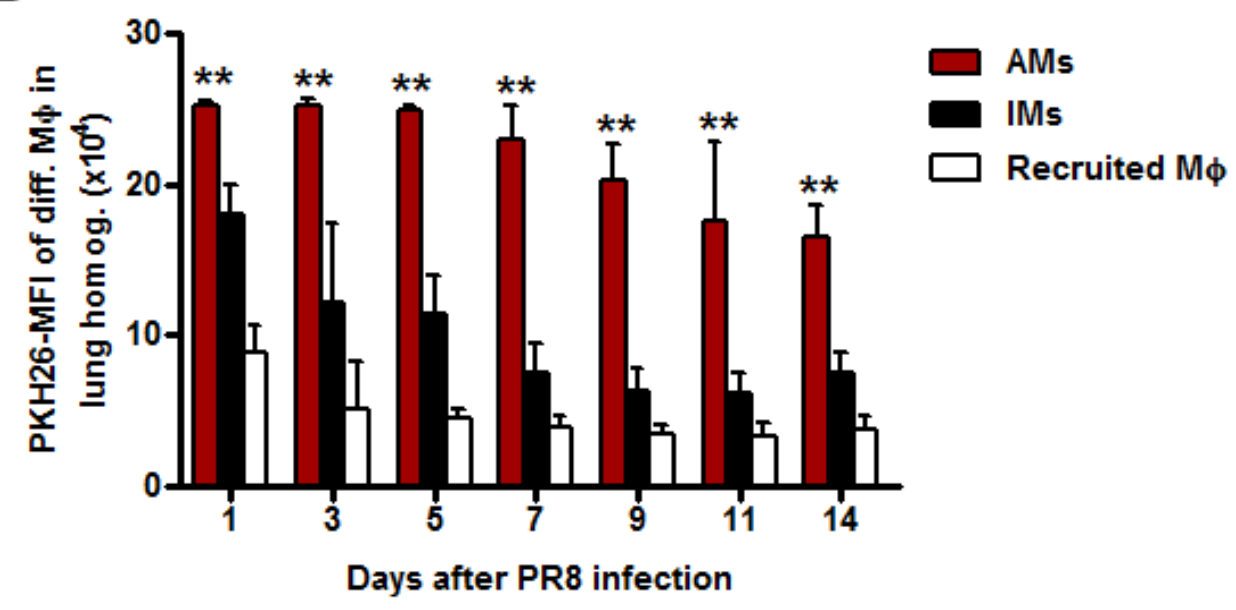

Figure 2-6. Differential mean fluorescence intensity of PKH26-PCL dye of different pulmonary macrophage subsets during influenza infection

Comparisons of MFI-PKH26 (mean fluorescence intensity of PKH26-PCL) of different pulmonary macrophage subsets, including resident AMs, IMs, and recruited macrophages in BALF (A) and post-lavage lungs (B) during the course of influenza infection ( $\mathrm{n} \geq 4$ ). ${ }^{*} P<0.05,{ }^{* *} P<0.01$ by Dunnett's multiple comparison test (ANOVA), compared with $\mathrm{AMs}$ at each time point. The bar graphs show the average $\pm \mathrm{SD}$. 
not express surface Ly6C, while resident IMs express intermediate levels, reflecting their intermediate stage of maturation and supporting the previous studies suggesting IMs as precursors for AMs $[172,196]$. In contrast, the recruited exudates macrophages with $\mathrm{CD} 11 \mathrm{~b}{ }^{\text {high }}$ phenotype showed significantly high expression levels of Ly6C (Figure 2-7).

We also determined the stability of PKH26-PCL dye in vivo in the mock-infected lungs, to exclude the possibility that these observed changes in PKH26-MFI of lungresident macrophages after influenza infection were not due to instability of the dye, but rather due to changes in resident macrophage pool size via maturation of unlabeled macrophages. PKH26-PCL dye demonstrated efficient labeling and stability inside the lung-resident macrophages for at least 14 days after mock-infection (i.e. 19 days after labeling), thereby covering the tracking period during which we did the kinetics analyses (Figure 2-8).

\section{Ontogeny of the Replenished Pool of Alveolar Macrophages after Influenza Infection}

To determine origin of the replenished AM pool during the resolution phase of influenza infection, we sub-gated AMs based on the fluorescence intensity of PKH26-PCL dye into PKH26 ${ }^{\text {high }}$, PKH26 ${ }^{\text {int }}$, and PKH26 subsets. We then measured the frequencies of these 3 sub-populations as percentages of total numbers of resident AMs. Surprisingly, the PKH26- subset did not virtually contribute to the replenishment of resident AM pool, implying a neglected contribution of the recruited, non-labeled, macrophages in resident AM homeostasis. Instead, more than $75 \%$ of AM pool after full replenishment was composed of PKH26 ${ }^{\text {high }}$ subset (on days 11 and 14 p.i), suggesting their probable origin from $\mathrm{PKH} 26^{\text {high }}$ IMs after differentiation and maturation into the resident AM phenotype (Figure 2-9).

The remaining portion of the replenished AMs consists of $\mathrm{PKH} 26^{\mathrm{int}}$ subset and represents about 25\% of total replenished AMs (Figure 2-9). Its intermediate level of PKH26-PCL fluorescence intensity suggests that it developed through proliferation of the remaining PKH26-labeled AMs or PKH26-labeled IMs followed by maturation into the AM phenotype.

\section{Influenza Infection Induces Cell Death of Alveolar Macrophages}

To determine whether AM depletion is due to a cell death process, we measured the total numbers of dead AM cells during PR8 infection using a cell viability dye (live/dead aqua). The total numbers of dead AMs were significantly higher in both BALF and post-lavage lungs of influenza-infected mice than in mock-infected mice (Figure 2-10A). In contrast, there was no significant difference between the total numbers of dead IMs in influenza-infected and mock-infected mice (Figure 2-10B). To determine the type of AM cell death process, we examined alveolar macrophages for any morphologic changes associated with influenza infection. Diff-Quick -stained cytospin slides of BALF 


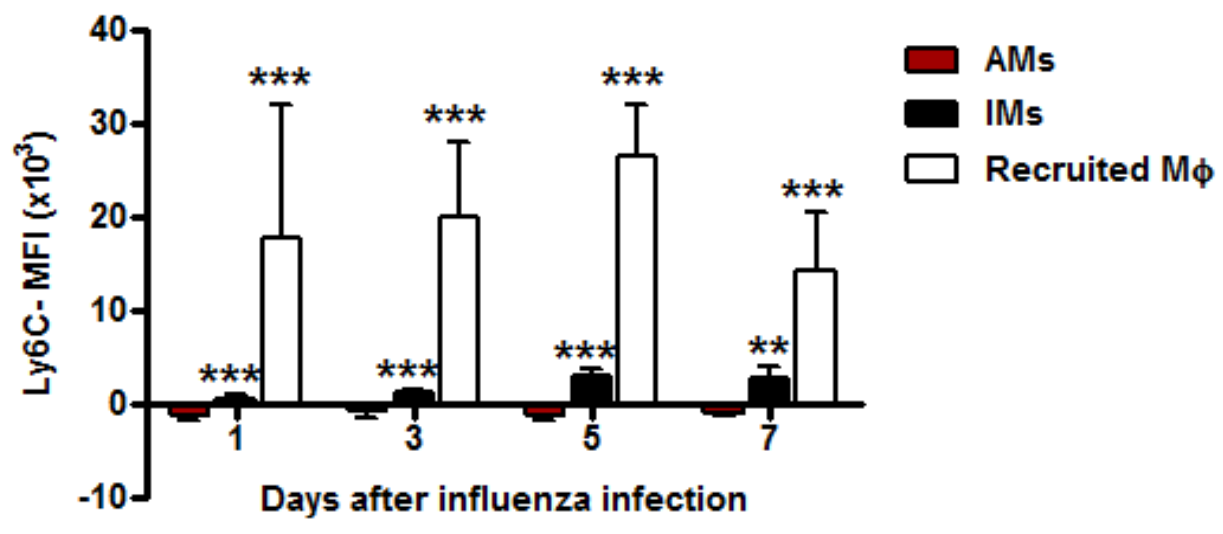

Figure 2-7. Differential surface expression of Ly6C marker on different pulmonary macrophage subsets

Ly6C-MFI are compared at different time points after influenza infection.

$* * P<0.01, * * * P<0.001$ by Mann-Whitney U test, where AMs are compared with IMs, and IMs are compared with recruited MФ at each time point $(n \geq 4)$. The bar graphs show the average \pm SD. 


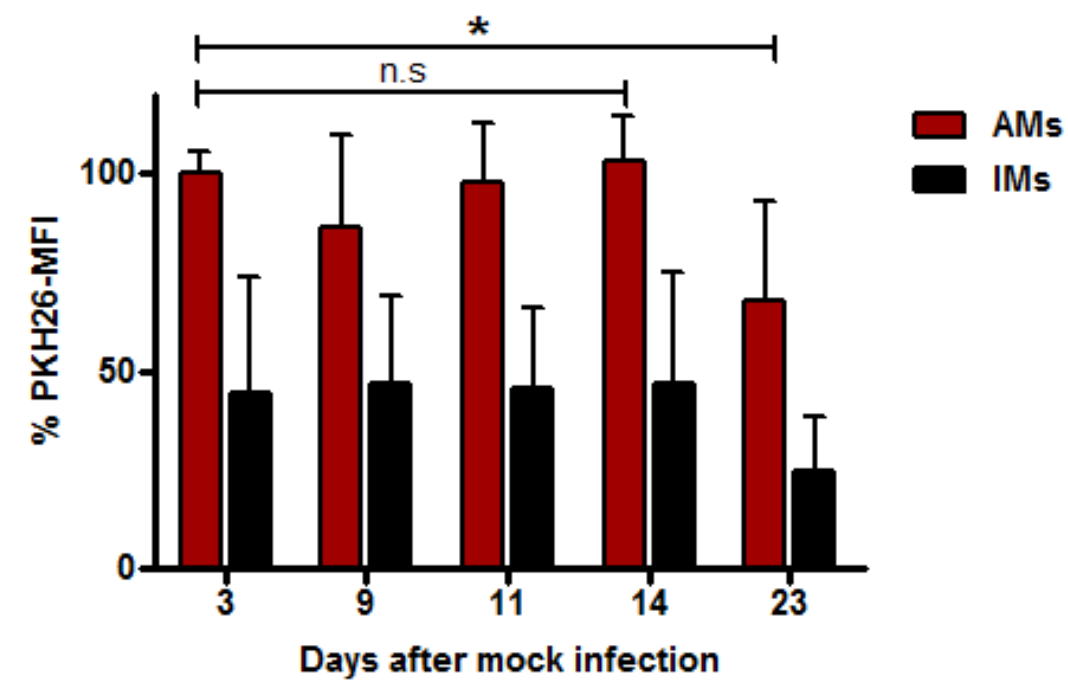

Figure 2-8. Fluorescence intensity of PKH26-PCL dye is stable in lung-resident macrophages for 2 weeks after mock infection

$\%$ PKH26-MFI values are calculated as percentages of the average PKH26-MFI of AMs on day 3 after PBS administration ( $\mathrm{n} \geq 4)$.

$* P<0.05$ by Dunnett's multiple comparison test (ANOVA), compared with \% PKH26MFI of AMs on day 3 after PBS. The bar graphs show the average \pm SD. 


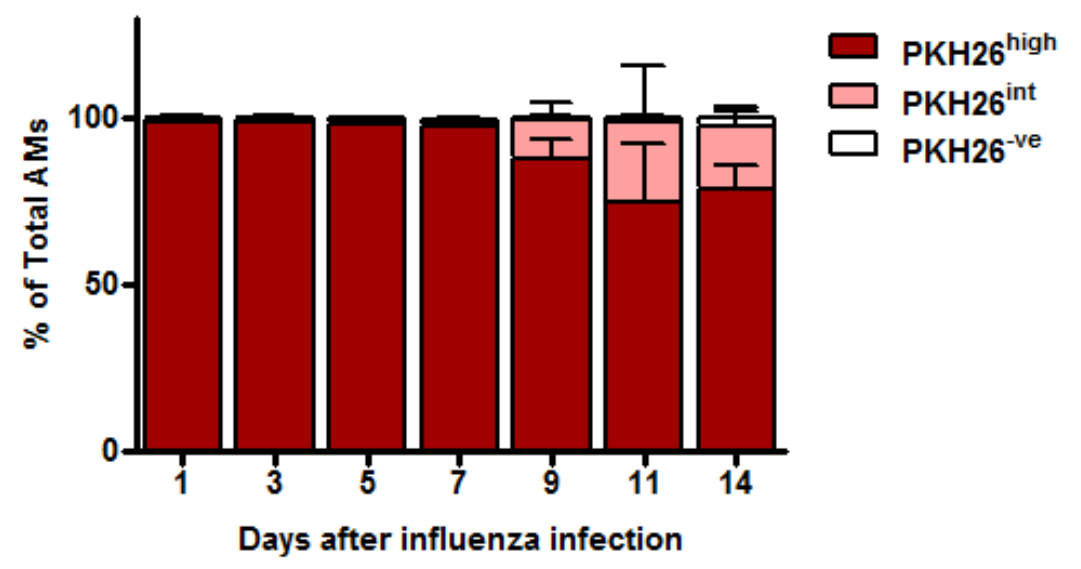

Figure 2-9. Ontogeny of the replenished alveolar macrophage pool during influenza infection

Alveolar macrophages were sub-gated based on degree of PKH26-PCL labeling into PKH26 ${ }^{\text {high }}, \mathrm{PKH}_{26} 6^{\text {int }}$, and PKH26- subsets. Then frequencies of each sub-population were measured as percentages of total numbers of AMs at different time points after PR8 influenza infection $(\mathrm{n}=6-8)$.

The bar graphs show the average \pm SD. 

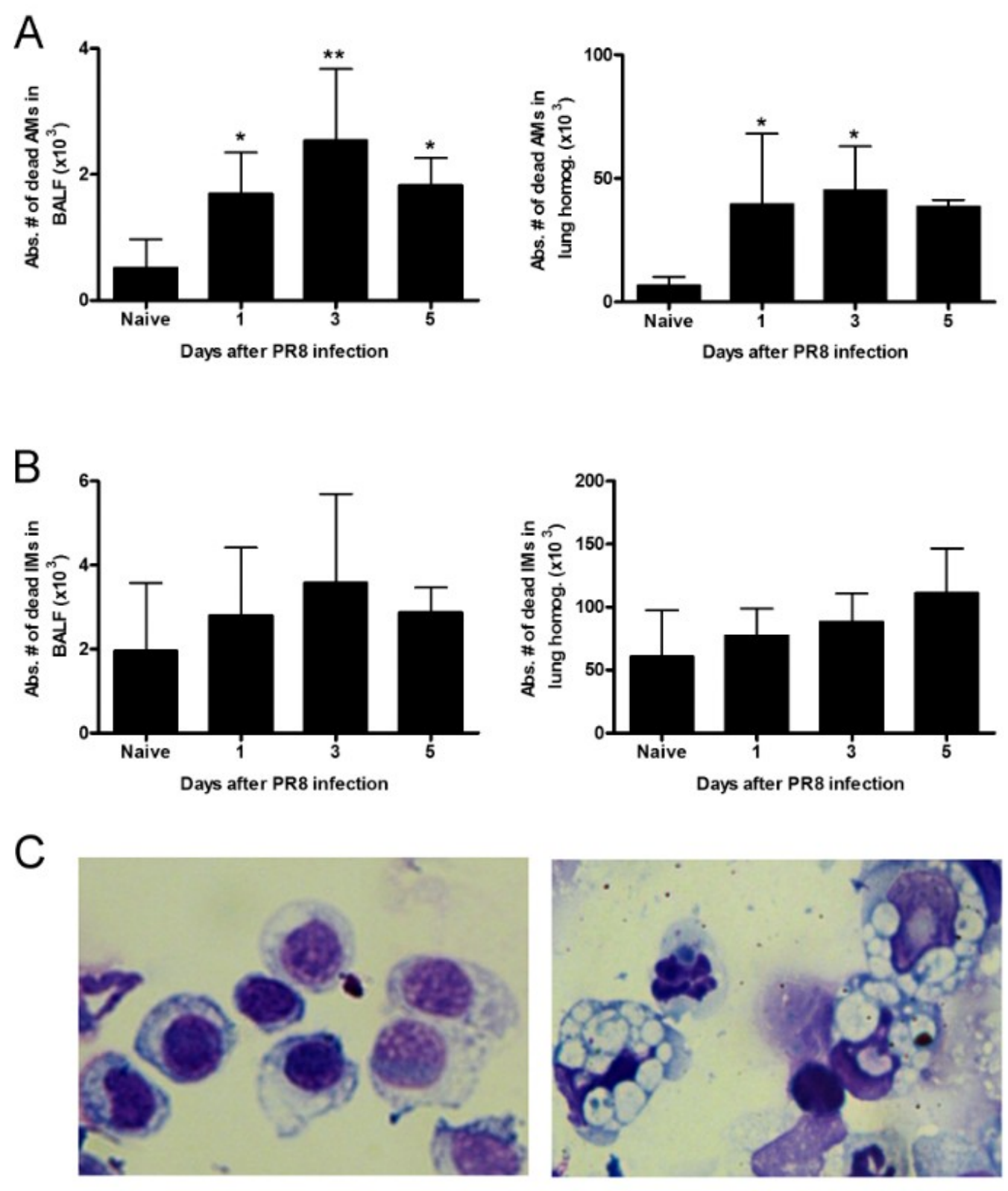

Mock-infected

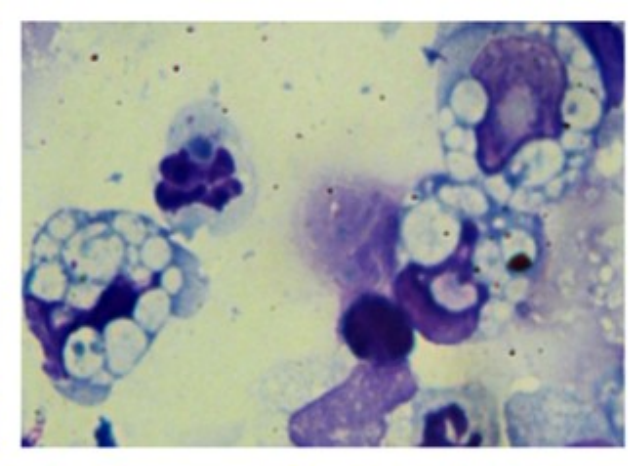

PR8-Infected (Day 3)

Figure 2-10. Influenza infection induces significant alveolar macrophage cell death

Total numbers of dead AMs (A) and IMs (B) are calculated during influenza infection. (C) Cytospin of Diff quick-stained BALF cells from mock-infected and PR8-infected mice 3 days p.i at magnification $\times 500$.

${ }^{*} P<0.05,{ }^{*} P<0.01$ by Dunnett's multiple comparison test (ANOVA), compared with mock-infected naive mice. The bar graphs show the average \pm SD. 
cells harvested from influenza-infected mice 3 days p.i. showed many macrophages with cellular damage manifestations. They were characterized by distorted nuclei and more vacuoles in cytoplasm than in mock-infected mice (Figure 2-10C). This suggests that cell death was due to a secondary necrotic process.

To determine if AMs were lost due to recruitment to the lung-draining lymph nodes, we examined the mediastinal lymph node 7 days after PR8 influenza infection and did not observe significant recruitment of PKH26-labeled AMs to it (data not shown).

\section{Discussion}

In this chapter, we determined the impact of sublethal influenza infections on the two major subsets of murine lung-resident macrophages in vivo. Dramatic depletion of resident AMs, but not IMs, takes place for a certain period during influenza infection, in association with a remarkable increase in the dead AM cell numbers early after infection. This depletion was not only observed during infection by a mouse-adapted H1N1 influenza A virus strain (PR8), but it was also shown during sublethal infections using human clinical isolates of the 2009 pandemic H1N1 influenza A virus and influenza B virus.

Tracking the PKH26-labeled AMs during PR8 influenza infection revealed a temporary depletion process from day 3 to day 9 after infection under the infection settings and with the mouse strain which we used. About $70 \%$ of AMs were depleted after 3 days, reaching a nadir ( $>90 \%$ depletion) 7 days after PR8 influenza infection. This matches the previously established boundaries for maximum synergism in the secondary bacterial infection model [197], which parallel the typical order and timing of infections in humans [198]. Interestingly, in another influenza infection model using a different strain, percentage of resident AMs - gated as CD1 $1 \mathrm{c}^{\text {high }}$ Mac- $1^{-}$- decreased on day 3 p.i [199], which is consistent with our findings. However, the authors did not show the absolute numbers of these cells.

Efficient replenishment of the AM pool then took place during the resolution phase of influenza infection. We investigated the ontogeny of the replenished AMs, and found that their pool was mainly recovered through local proliferation and differentiation of IMs into AM phenotype. Strikingly, our novel findings were also recently suggested by another research group that used a genetic approach to track genetically labeled lungresident macrophages during PR8 influenza infection. They showed significant lungresident macrophage cytoablation on day 6 after influenza infection and suggested that these macrophages could repopulate later, mainly by local proliferation [173].

We highlighted the critical requirement for using approaches that could efficiently distinguish between different lung-resident macrophage subsets - AMs and IMs, and differentiate them from the inflammatory macrophages and DCs recruited during lung infections. Indeed, the PKH26-PCL in vivo labeling approach combined with the flow cytometric analyses and confocal microscopic examination could efficiently label and 
characterize different subsets of lung-resident macrophages in the lungs of naïve mice, in addition to differentiating them from recruited unlabeled immune cells. Therefore, one observation stemming from our findings is that examination of lung-resident macrophages in the setting of influenza infection should be cautiously performed. During influenza infection, diverse chemokines are upregulated in lungs, activating an influx of heterogeneous populations of innate immune cells, such as monocytes, inflammatory macrophages, and monocyte-derived dendritic cells. The dynamic changes in the phagocyte populations in influenza-infected lungs require critical methods to distinguish resident macrophages from recruited ones. Based on the surface phenotype of lungresident macrophages in naïve mice, we used an extensive gating strategy to analyze and differentiate resident and recruited macrophages. Thus, AMs were gated as CD11 $\mathrm{c}^{\text {hi }}$ $\mathrm{F} 4 / 80^{\text {hi }} \mathrm{CD} 11 \mathrm{~b}$, while IMs and recruited macrophages were gated as CD11 $\mathrm{c}^{\text {hi }} \mathrm{F} 4 / 80^{\text {hi }}$ $\mathrm{CD} 11 \mathrm{~b}^{\text {low-int }}$ and CD11 $\mathrm{c}^{\text {hi }} \mathrm{F} 4 / 80^{\text {hi }} \mathrm{CD} 11 \mathrm{~b}^{\text {high }}$ respectively. Further characterization showed the ability of the Ly6C marker to differentiate between resident and recruited phagocytes and their maturation stage in influenza-infected lungs. Ly6C antigen is expressed by circulating blood monocytes and macrophages, which can be recruited to tissues under inflammation conditions [191]. However, Ly6C expression is down-regulated during differentiation of blood monocytes or macrophages into tissue resident macrophages after migration to tissue. Therefore, it can be added as a suitable marker to differentiate macrophage subsets and their stages of maturation into tissue resident phenotype [192]. Moreover, in vivo labeling of lung-resident macrophages was done to validate our gating strategy and to distinguish between resident AMs and recruited macrophages. The PKH26-PCL dye used to label macrophages forms fluorescent microparticles, which can be taken up by the resident phagocytes in the lungs. The dye remains stable intracelullarly for more than 21 days [101], and emits high fluorescence intensity for at least 19 days based on our findings.

Furthermore, monitoring changes of MFI-PKH26 for lung-resident macrophages reflected the dynamic changes in their absolute numbers during influenza infection. For instance, expansion of the AM pool during the recovery phase was accompanied by significant decrease in their MFI-PKH26. Nevertheless, the AM population showed the highest MFI-PKH26 at various times during the first 2 weeks after influenza infection. Thereby, the significantly high MFI-PKH26 of AMs during their replenishment phase in addition to the high percentages of $\mathrm{PKH} 26^{\text {high }}$ sub-populations implied that the AM pool was mainly replenished via local proliferation and differentiation of the PKH26-labeled IMs into an AM surface phenotype, rather than maturation of PKH26-unlabeled recruited blood macrophages. In contrast, the quick drop in MFI-PKH26 of IMs in the first week after influenza infection, without apparent changes in their absolute cell numbers, suggests that influenza infection may induce partial depletion of the IM pool. However, this partial depletion can be quickly re-compensated by the proliferative capacity of IMs, maturation of the recruited macrophages, or both [94, 172].

Several investigators, who used animal models of influenza infections, couldn't identify depletion of resident AMs during influenza infections because of some limitations in their analysis. Mostly they used gating strategies not sufficient to distinguish between resident AMs, IMs, and recruited exudate macrophages. For 
example, gating for a single marker like F4/80 antigen or macrophage-specific esterase staining of macrophages isolated from BAL fluid during influenza infection is targeting mixed populations of resident and recruited macrophages whose total cell numbers increase during infection [200]. In addition, although the same in vivo labeling method was used before, AM depletion couldn't be identified during influenza [101]. Based on their analysis, they gated AMs as $\mathrm{CD} 11 \mathrm{c}^{+} \mathrm{PKH} 26^{+}$which may be mixed with IMs. They analyzed AM numbers on day 9 after influenza infection in BAL fluid only. Based on our model, we showed certain period for AM depletion from day 3 to day 9 after influenza infection. Analyzing depletion events by monitoring AMs at a single time point (day 9 p.i) during influenza infection is inconclusive. Furthermore, analyzing cells in BAL fluid may cause us to miss AMs that remain adherent in the lungs after lavage leading to the underestimation of the resident AM pool size during the comparison between influenzainfected and mock-infected mice. Likewise, in another study the authors did not show AM depletion because of missing CD11b marker in their AM gating, as well as harvesting only BAL fluid for kinetics analysis [185]. Taken together, missing an important marker that distinguishes between different lung-resident macrophage subsets and recruited monocytic cells or using BAL fluid only for analyzing resident AMs may lead to erroneous conclusions regarding their kinetics during infections.

In summary, sublethal influenza infection induces transient depletion of the resident AMs, which temporally parallels the period of the increased clinical susceptibility to SBP following influenza infections in humans. These findings led us to propose a novel mechanism of influenza-mediated immunosuppression in the lungs. However, whether this proposed mechanism contributes to the increased vulnerability to SBP still needs to be experimentally tested. 


\section{CHAPTER 3. DEPLETION OF RESIDENT ALVEOLAR MACROPHAGES DURING INFLUENZA INFECTION CONTRIBUTES TO ENHANCED SUSCEPTIBILITY TO BACTERIAL PNEUMONIA ${ }^{2}$}

\section{Introduction}

Bacterial super-infections are among the common complications during influenza infections. Specifically, SBP has been found complicating significant percentages of severe cases and deaths during periods of influenza outbreaks and seasonal influenza. Furthermore, SBP remarkably contributed to the increased mortality during several influenza pandemics even after antibiotics discovery and their wide routine use. For instance, in the last 2009 influenza pandemic, $25 \%-50 \%$ of severe or fatal cases had SBP $[35,36,126]$.

Mechanisms of the increased predisposition to SBP following influenza infections have been investigated for a long time [35, 147, 201]. Several studies have been performed to explain the synergistic interactions between influenza viruses and secondary bacterial pathogens. Previous studies of viral-bacterial synergism in our laboratory have focused on influenza virus virulence factors and their damaging effects on respiratory tract epithelial cells, together with synergistic inflammatory lung injury during coinfection $[35,129]$. However, our findings in Chapter 2 provided insights of a novel mechanism underlying the increased permissiveness to SBP through influenza-induced loss of the most critical innate immune cells in the lungs - the resident AMs.

Lung-resident AMs are considered the most important cells of the first line of innate defense against respiratory pneumococcal infections [93] due to their high phagocytic capacity [94-96]. We reasoned that early escape from the first line of defense in the lungs could have profound effects on immunity to a variety of pathogens, including secondary bacterial invaders. Enhanced bacterial growth and replication through this mechanism could allow enhanced expression of virulence factors and the resulting inflammatory response. Therefore, we sought to determine if the resident AM depletion during influenza infection contributes to the enhanced susceptibility to SBP.

Additionally, we tested an immunomodulation intervention to prevent this defect in the antibacterial immunity by expanding the lung-resident macrophage pool during influenza infection so that it might protect influenza-infected mice against SBP.

We used standard murine influenza virus infection and co-infection models [202], in which BALB/c female mice are infected intranasally by a sublethal dose of influenza A virus - this primary infection may then be followed by a sublethal dose of $S$. pneumoniae at different time points after influenza infection. We demonstrated that

${ }^{2}$ Adapted with permission from The American Association of Immunologists, Inc. Ghoneim HE, Thomas PG, McCullers JA. Depletion of Alveolar Macrophages during Influenza Infection Facilitates Bacterial Superinfections. J Immunol 2013; 191:1250-9. 
resident AM depletion impairs early bacterial clearance, supporting development of SBP during the AM depletion phase. These data have implications for understanding virusinduced host immune suppression that may lead to improved prevention and treatment of primary or secondary infections in the lungs.

\section{Materials and Methods}

\section{Infectious Agents}

For influenza infections, we used PR8 influenza virus and prepared the infectious dose from the same stocks as described previously (page 29).

For secondary bacterial infections, we used the clinical human isolate of $S$. pneumoniae A66.1, a type 3 encapsulated strain. It was engineered to express luciferase (Kevin Francis and Jun Yu, Xenogen Corporation, Alameda, CA). Pneumococci were grown in Todd Hewitt broth (Difco Laboratories, Detroit, MI) to an OD620 of approximately 0.4 and then frozen at $-80^{\circ} \mathrm{C}$ mixed $2: 1$ with $5 \%$ sterile glycerol. The titers of the frozen stocks were quantitated on tryptic soy agar (Difco Laboratories, Detroit, MI) supplemented with 3\% v/v sheep erythrocytes (blood agar). In all instances, the infectious dose administered was confirmed by serial dilution and plating of the bacterial suspension on blood agar plates.

\section{Mice}

Six- to 8-week-old female BALB/c mice (Jackson Laboratory, Bar Harbor, ME) were maintained in a Biosafety Level 2 facility in the Animal Resource Center at St. Jude. Animals were given general anesthesia that consisted of $2.5 \%$ inhaled isoflurane (Baxter Healthcare Corporation, Deerfield, IL) prior to all interventions, and all studies were approved by the Animal Care and Use Committee at St. Jude.

\section{Infectious Model}

Infectious agents were diluted in sterile PBS and administered intranasally in a volume of $100 \mu \mathrm{l}(50 \mu \mathrm{l}$ per nostril) to anesthetized mice held in an upright position. In all experiments of influenza infection, PR8 influenza virus was given at a dose of 25 TCID50 per $100 \mu 1$ per mouse, which did not cause mortality when given alone. In coinfection experiments, PR8 infection was followed at the specified time point by pneumococcal challenge with 200 colony forming units (CFUs) per mouse. Infected mice were weighed and assessed daily for illness and mortality for 7 days after pneumococcal challenge. 


\section{Imaging of Live Mice}

Mice were then imaged for 60 seconds using an IVIS CCD camera (Caliper Life Sciences, Alameda, CA) daily after pneumococcal challenge to monitor in vivo pneumococcal pneumonia development. Total photon emission from selected and defined areas within the images of each mouse was quantified using Living Image software (Caliper Life Sciences, Alameda, CA) as described previously [152, 203, 204] and expressed as the flux of relative light units per minute. Pneumonia was defined as visible bioluminescence within the thorax and detection of a flux of $>11,000$ relative light units per minute.

\section{Flow Cytometric Analysis of Immune Cells in BALF and Post-lavage Lungs}

Following euthanasia by $\mathrm{CO}_{2}$ inhalation, the trachea was exposed and cannulated with a 24-gauge plastic catheter (Becton Dickinson Infusion Therapy Systems, Inc., Sandy, UT). Lungs were lavaged 4 times with $1 \mathrm{ml}$ of cold sterile HBSS supplemented with $0.1 \mathrm{mM}$ EDTA. The whole lungs after lavage were harvested and processed, in addition to BALF cell suspension, for surface marker staining as described previously (page 30). AMs and IMs were gated as described previously (page 30). Viable and nonviable cells were counted before surface marker staining, and the percentage viability was counted via the trypan blue exclusion method using a Cell Countess System (Invitrogen, Grand Island, NY). The absolute numbers of different cell types were calculated based on the proportion of viable events analyzed by flow cytometry as related to the total number of viable cells per sample.

\section{Determination of Early Pneumococcal Clearance}

Early pneumococcal clearance was determined by measuring the pneumococcal count remaining within alveolar airspaces. Briefly, mice were euthanized by $\mathrm{CO}_{2}$ inhalation $3 \mathrm{~h}$ after pneumococcal inoculation. BALF was harvested using sterile HBSS supplemented with $0.1 \mathrm{mM}$ EDTA (lavage by $1 \mathrm{ml}$ twice), and then half the BALF was spread on blood agar plates supplemented with $0.4 \mathrm{mg}$ kanamycin $/ \mathrm{ml}$ blood agar to select for kanamycin-resistant luciferase-expressing pneumococci [203] and incubated at $37^{\circ} \mathrm{C}$ overnight.

\section{Local GM-CSF Treatment Regimen}

We treated anesthetized mice intranasally with $25 \mu \mathrm{g}$ of recombinant mouse GMCSF (Granulocyte/Monocyte-Colony Stimulating Factor, Invitrogen, Grand Island, NY) in $100 \mu \mathrm{l}$ of sterile PBS on days -1 and +1 before and after PR8 infection. We treated control mice with vehicle (PBS) only. 


\section{Statistical Analysis}

Comparison of survival and pneumonia development between groups of mice was done with the log-rank chi-squared test on the Kaplan-Meier survival data. Comparison of bacterial titers, and cell counts in BALF, and post-lavage lung homogenate between groups were compared using analysis of variance (ANOVA). Comparison of weight loss between groups of mice was done using Mann-Whitney $U$ test for pair-wise comparisons. A p-value of $<0.05$ was considered significant for these comparisons. Prism 4 for Windows (GraphPad Software, Inc., V 4.03) was used for all statistical analyses.

\section{Results}

\section{Influenza Infection Enhances Susceptibility to Secondary Pneumococcal Pneumonia}

Influenza-mediated death of AMs is likely to have significant effects on primary and secondary immunity. To explore one potential defect, we studied the permissiveness of influenza-infected hosts to secondary respiratory bacterial infections. We first infected $\mathrm{BALB} / \mathrm{c}$ female mice intranasally with PR8 influenza virus using a sublethal dose (25 TCID50). Influenza-infected mice showed mild morbidity manifested as loss of about $10 \%$ of their original body weight within 7 days after influenza infection (Figure 3-1A). At that time point, we induced secondary bacterial infection via intranasal administration of a small inoculum (200 CFUs) of the serotype 3 clinical isolate of $S$. pneumoniae, A66.1. Influenza-infected mice showed high susceptibility to secondary pneumococcal infection with a significant increase in morbidity and continuous body weight loss (Figure 3-1A). All co-infected mice died within 3 to 5 days after pneumococcal inoculation (Figure 3-1B). In contrast, all single influenza- or single pneumococcusinfected mice recovered quickly with no mortality (Figure 3-1A and B). To determine whether the co-infected mice died due to pneumococcal pneumonia, we monitored pneumococcal growth in vivo through bioluminescent imaging of the lungs [203]. Only co-infected mice developed serious pneumococcal infections; pneumonia occurred within $48 \mathrm{~h}$ after pneumococcal inoculation (Figure 3-1C). Likewise, sublethal influenza B virus infection enhanced the susceptibility to develop lethal secondary pneumococcal pneumonia, if influenza-infected mice get secondarily infected with 200 CFUs of pneumococci at time points with significant AM depletion (data not shown).

\section{AM Depletion during Influenza Infection Impairs Early Pneumococcal Clearance}

To determine whether the observed depletion of the AM pool contributes to enhanced susceptibility to secondary pneumococcal infection in influenza-infected hosts, AM phagocytic function was assessed in vivo by measuring early pneumococcal clearance with or without influenza infection. First, we determined the earliest time point at which a small pneumococcal inoculum (200 CFUs) could be efficiently cleared within the alveolar airspaces of naive mice. Pneumococcal early clearance was tested at different 
A

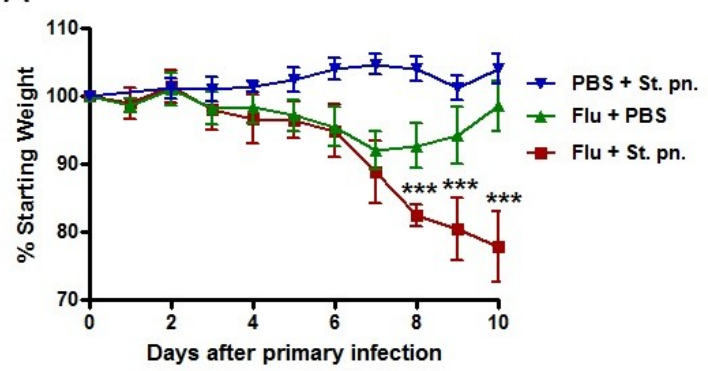

C

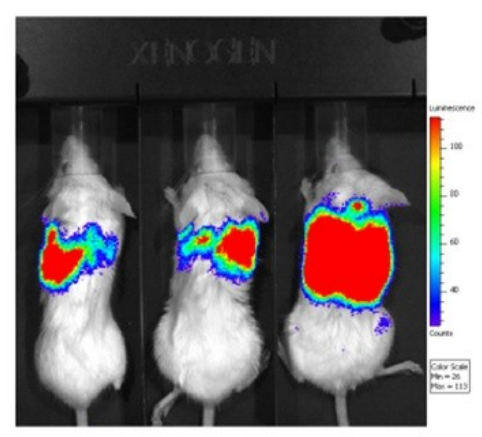

B

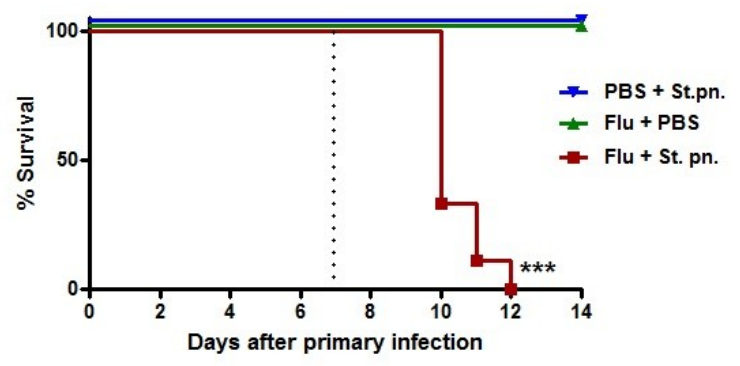

D

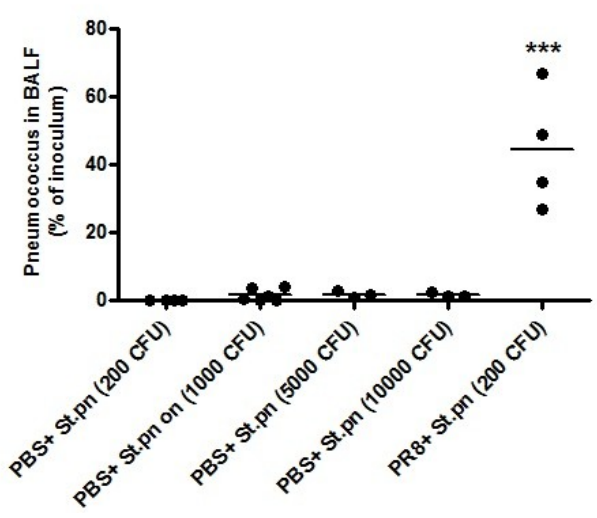

Figure 3-1. Murine influenza-pneumococcal co-infection model

Body weight loss (A) and survival rate (B) of single pneumococcal-infected (PBS and $S$. pneumoniae (St. pn.), $\mathrm{n}=6$ ), single influenza-infected (influenza and PBS, $\mathrm{n}=8$ ), and co-infected (influenza and $S$. pneumoniae, $\mathrm{n}=9$ ) mice with 7 days interval between two doses in each group. (C) Thorax bioluminescence of luciferase-expressing A66.1 pneumococcus from 3 representative co-infected mice showing development of pneumococcal pneumonia. Images were taken $48 \mathrm{~h}$ after secondary bacterial challenge using 200 CFUs of pneumococcus on day 7 after influenza infection. (D) Pneumococcal titers harvested $3 \mathrm{~h}$ after bacterial inoculation (inoculum of 200 or more CFUs) from alveolar airspaces of mock-infected and influenza-infected mice 7 days p.i. are shown as percentage of inoculum.

*** $P<0.001$ by Mann-Whitney $\mathrm{U}$ test, compared at each time point with single influenza-infected mice group (Panel A), by log-rank test on the Kaplan Meier survival data (Panel B), or by Dunnett's multiple comparison test (ANOVA), compared with mock-infected mice (Panel D). Data represent the average \pm SD. 
time points $(1,2,3$, and $4 \mathrm{~h})$ after bacterial inoculation. Complete pneumococcal clearance was observed within $3 \mathrm{~h}$ after pneumococcal inoculation in naïve mice (data not shown). Then, we tested the ability of influenza-infected lungs to clear this small dose of pneumococcus (200 CFUs) at this early time point. This experiment demonstrated that early pneumococcal clearance was significantly impaired in the alveolar airspaces of influenza-infected mice compared with mock-infected controls. Mock-infected mice could efficiently clear more than $95 \%$ of up to 50 -fold higher doses of pneumococcus, while influenza virus-infected mice were unable to clear the basal inoculum (Figure 3-1D).

\section{Influenza-infected Mice Demonstrate Increased Susceptibility to Secondary Pneumococcal Pneumonia during the AM Depletion Phase}

To determine whether AM depletion during influenza infection correlates with the susceptibility to secondary pneumococcal infection, we examined the early pneumococcal clearance at different time points after influenza infection. Early pneumococcal clearance was significantly impaired during PR8 infection from 3 until 9 days p.i. (Figure 3-2A). This period of early pneumococcal clearance impairment closely mirrors the phase of the AM pool depletion observed during PR8 infection (Figure 2-4A and B). Later, after the full replenishment of the AM pool, the impaired early pneumococcal clearance was restored starting from day 11 p.i (Figure 3-2A).

To determine whether the impaired early pneumococcal clearance during influenza infection enhances susceptibility to secondary pneumococcal pneumonia development, we monitored mouse lungs via bioluminescence imaging $24 \mathrm{~h}$ after bacterial inoculation. Among the influenza-infected mice, 100\% developed secondary pneumococcal pneumonia when they were secondarily infected by pneumococcus 3,5 , or 7 days after influenza infection. In contrast, all influenza-infected mice that were secondarily infected either 1 days or 14 days p.i. cleared the bacterial dose efficiently and did not develop secondary pneumococcal pneumonia (Figure 3-2B). Secondary pneumococcal pneumonia development was less frequent in influenza-infected mice that were secondarily infected by pneumococcus on 9 or 11 days p.i., with only $60 \%$ or $40 \%$ of pneumonic mice, respectively (Figure 3-2B). As expected in this model, all coinfected mice that developed secondary pneumococcal pneumonia died within few days due to bacterial pneumonia (Figure 3-2C).

\section{Local GM-CSF Treatment Expands the Lung-resident Macrophage Pool in Influenza-infected Mice}

To restore the early bacterial clearance efficiency that was impaired during the AM depletion phase, we tested local recombinant GM-CSF treatment as a means to accelerate replenishment of the depleted AM pool in influenza-infected mice.

Recombinant GM-CSF was intranasally administered in 2 doses on days -1 and +1 before and after PR8 infection (Figure 3-3A). First, we analyzed the effect of this 


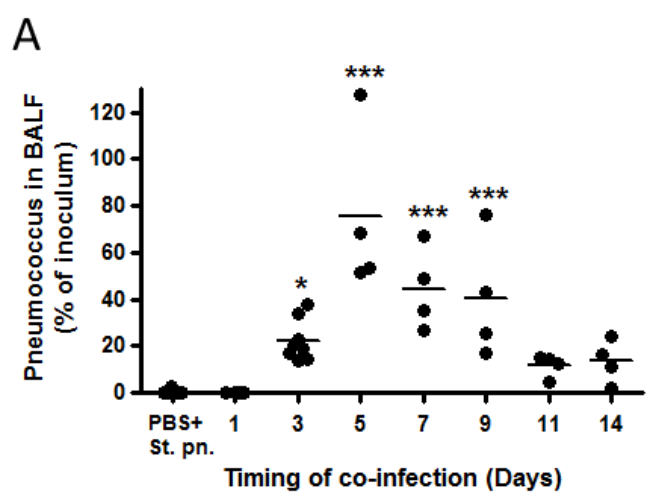

B

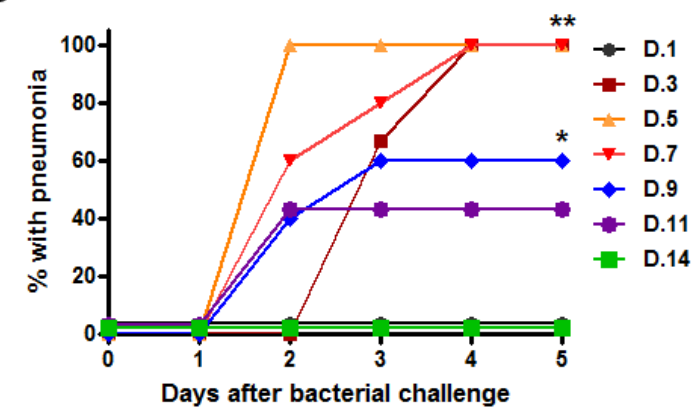

C

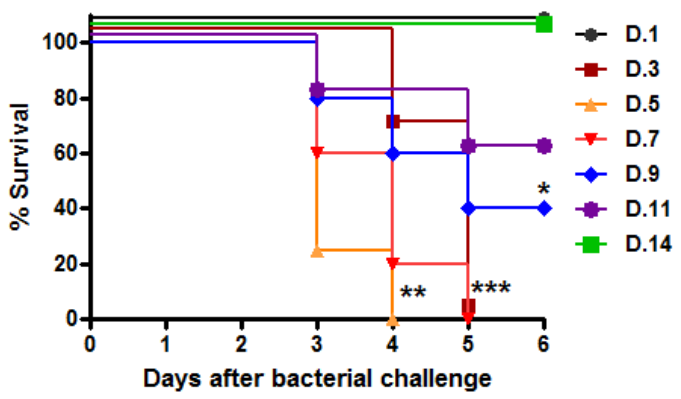

Figure 3-2. Successful co-infection synergism during the AM depletion phase in influenza-infected mice

(A) Pneumococcal CFUs, as percentages of inocula, harvested $3 \mathrm{~h}$ after bacterial inoculation (inoculum of $200 \mathrm{CFUs}$ ) from the alveolar airspaces of mock-infected and influenza-infected mice 1, 3, 5, 7, 9, 11, and 14 days p.i. Secondary pneumococcal pneumonia development (B) and mortality (C) are manifested in influenza-infected mice that are secondarily pneumococcal-infected during the AM depletion phase (D.1 group; $n$ $=5$, D. 3 group, $\mathrm{n}=6$; D.5 group, $\mathrm{n}=4$; D.7 group, $\mathrm{n}=10$; D.9 group, $\mathrm{n}=5$; D.11 group, $\mathrm{n}=5$; and D.14 group, $\mathrm{n}=5$ ).

$* P<0.05, * * P<0.01, * * * P<0.001$ by Dunnett's multiple comparison test (ANOVA), compared with mock-infected mice (Panel A), or compared with Day 1 or Day 14 coinfection groups by log-rank test on the Kaplan Meier survival data (Panel B and C). 
A

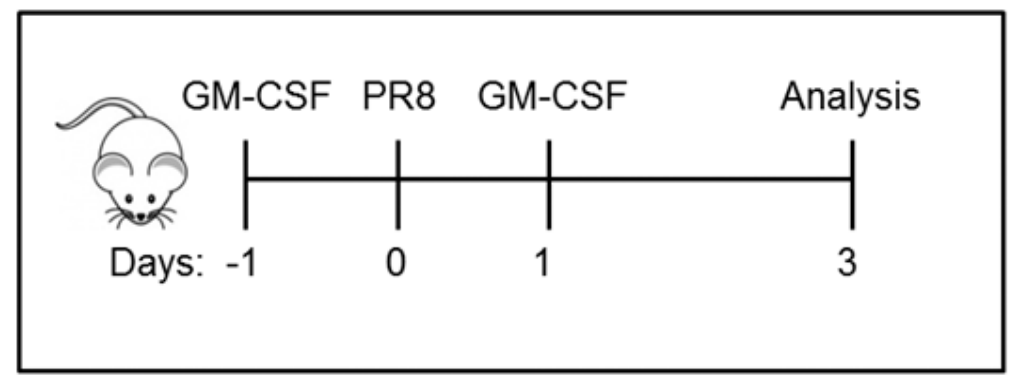

B
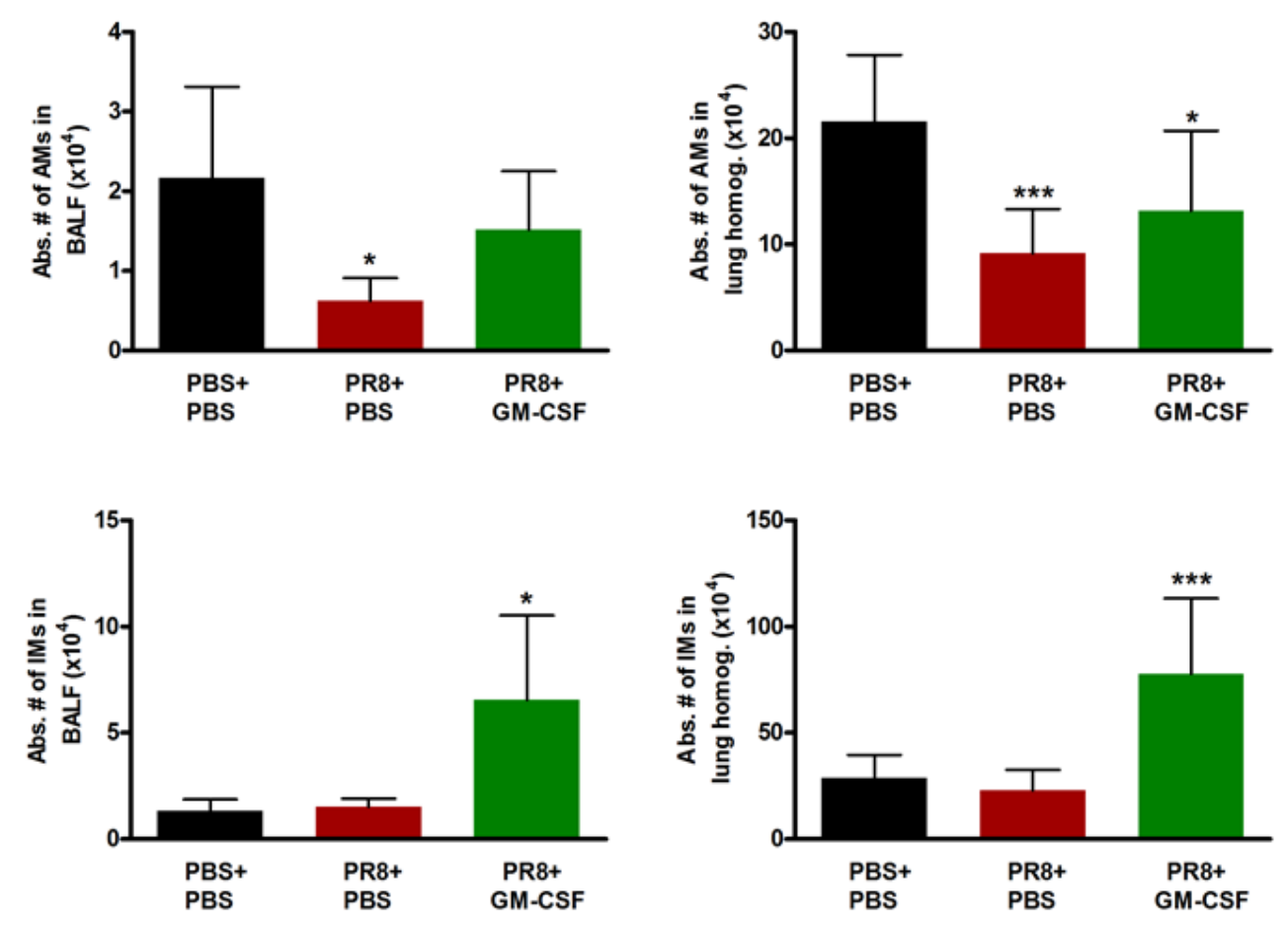

Figure 3-3. Local GM-CSF treatment expands the lung-resident macrophage pool in influenza-infected mice

(A) Diagram showing local GM-CSF treatment regimen, where recombinant GM-CSF was intranasally administered into PR8-infected mice on days -1 and +1 before and after infection. Then BALF and post-lavage lungs were harvested for analysis of lung-resident macrophages. (B) The absolute numbers of AMs and IMs increased in GM-CSF-treated influenza-infected mice analyzed 3 days p.i $(\mathrm{n} \geq 4)$. ${ }^{*} P<0.05,{ }^{* * *} P<0.001$ by Dunnett's multiple comparison test (ANOVA), compared with mock-treated mock-infected mice. The bar graphs show the average \pm SD. 
GM-CSF treatment regimen on the size of the AM and IM pools. Local GM-CSF treatment in influenza-infected mice resulted in a significant expansion of the IM pool with partial replenishment of the AM pool (Figure 3-3B).

\section{Local GM-CSF Treatment Decreases the Susceptibility to Secondary Pneumococcal Pneumonia Following Influenza}

To evaluate the efficacy of local GM-CSF treatment in the co-infection model, pneumococcus was administered 3 days after PR8 infection into GM-CSF-treated and mock-treated mice groups (Figure 3-4A). Local GM-CSF treatment led to better early pneumococcal clearance in influenza-infected mice than in mock-treated mock-infected mice. Conversely, early pneumococcal clearance remained impaired in mock-treated, influenza-infected mice (Figure 3-4B). Interestingly, some of the GM-CSF-treated influenza-infected mice could efficiently clear the bacterial inoculum, while others could not. Next, we tested the ability of local GM-CSF treatment to prevent secondary pneumococcal pneumonia development after bacterial inoculation 3 days p.i. Local GMCSF treatment protected more than $50 \%$ of co-infected mice against secondary pneumococcal pneumonia. Meanwhile, all mock-treated co-infected mice developed pneumococcal pneumonia (Figure 3-4C).

\section{Discussion}

Influenza is well known to increase susceptibility to secondary bacterial infections, such as SBP. The increased permissiveness of influenza-infected lungs to pneumococcal outgrowth suggests a defect in the host innate immune defenses that establishes a niche for bacterial infections. In this Chapter, we examined a novel mechanism of influenza-mediated immune suppression, which is the depletion of resident AMs. The resulting immune defect is likely to have pleiotropic effects on primary and secondary immunity. We examined one potential effect; bacterial escape from early innate immunity contributing to enhanced vulnerability to SBP. Resident AMs are essential for early bacterial clearance and protection against bacterial infections [93, 101]. We found that early clearance of small pneumococcal inocula was significantly impaired during the AM depletion phase. The impaired early bacterial clearance correlated with successful progression to SBP.

In most studies of primary or secondary pneumococcal pneumonia in animal models, AM-mediated protection has not been rigorously studied [101, 147, 205]. This appears mainly to be because of the use of high doses of pneumococcus $\left(\geq 1 \times 10^{5}\right.$ CFUs) to overwhelm the phagocytic capacity of resident AMs and induce a robust and reproducible pneumonia. Under these settings, neutrophil influx coupled with the development of early adaptive response in the form of serotype-specific antibody was found in these models to be important for host protection against primary pneumococcal pneumonia $[84,104,161]$. However, in the perhaps more physiologically relevant setting of a relatively small dose of pneumococcus (200 CFUs) after sublethal mild influenza 
A

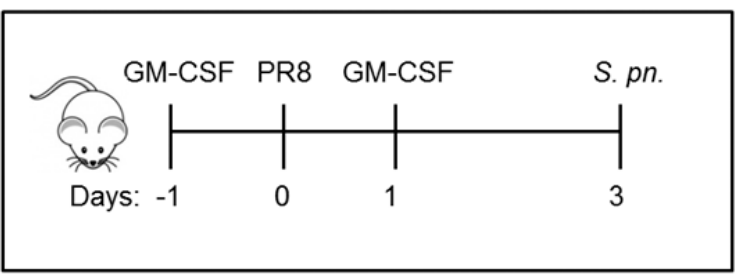

B

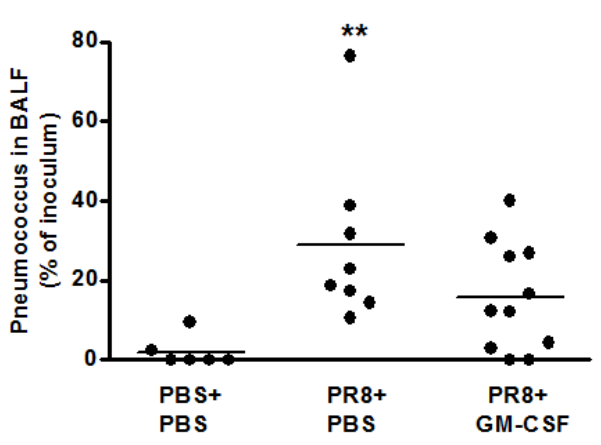

C

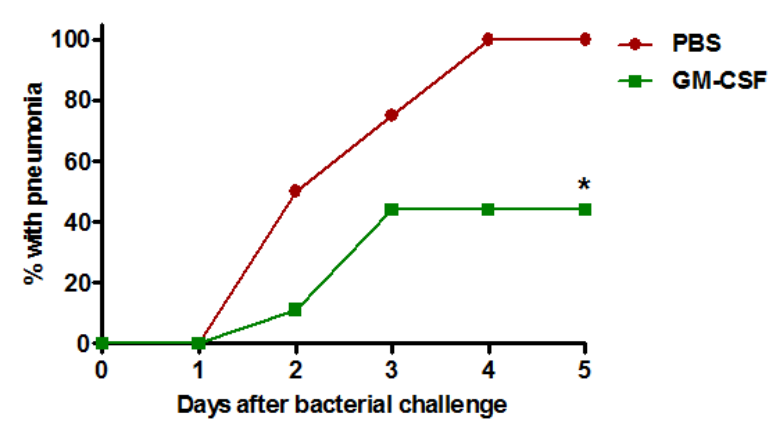

Figure 3-4. Local GM-CSF treatment decreases the susceptibility to secondary pneumococcal pneumonia following influenza

(A) Diagram showing local recombinant GM-CSF treatment into PR8-infected mice on days -1 and +1 before and after infection, followed by intranasal administration of 200 CFUs of pneumococcus on day 3. GM-CSF treatment improved early pneumococcal clearance within the alveolar airspaces $(\mathrm{B})$ and secondary pneumococcal pneumonia development (C) compared with mock-treated co-infected mice (GM-CSF-treated group, $\mathrm{n}=9$, PBS-treated group, $\mathrm{n}=4$ ).

$* * P<0.01$ by Dunnett's multiple comparison test (ANOVA), compared with mocktreated mock-infected mice (Panel B), or $* P<0.05$ by log-rank test on the Kaplan Meier survival data, compared with mock-treated co-infected mice (Panel C). 
infection, we could demonstrate a strong effect mediated by AMs. This low bacterial inoculum successfully caused lethal secondary pneumococcal pneumonia within 48-72 h after bacterial inoculation, pneumonia that was mainly dependent on an absence of the resident AMs.

GM-CSF is a cytokine with diverse functions and is known to be critical for effective innate immunity in lungs. It regulates AM differentiation and activation [188, 206], enhances proliferation of resident pulmonary macrophages [173, 192], and expands the pool of resident AMs [207]. It also has an important role in pulmonary surfactant homeostasis [208]. Recent studies showed that GM-CSF over expression in lungs has prophylactic activity against lethal influenza and pneumococcal pneumonias [207, 209, 210]. We found that local recombinant GM-CSF treatment in influenza-infected mice induced significant expansion of the IM pool. In addition, absolute numbers of AMs increased under this treatment regimen in influenza-infected mice. This increase was less than that of IMs, possibly because they were analyzed at an earlier time point (3 days p.i). Our data are consistent with previous studies showing that GM-CSF enhances the proliferation capacity of lung-resident macrophages and maturation of AMs. Considering the higher proliferative capacity of IMs over AMs, our short treatment regimen could significantly expand the pool of IMs more than AMs. As a result of the partial replenishment of the AM pool with pulmonary GM-CSF treatment, influenza-infected mice had partial restoration of efficient early pneumococcal clearance. Furthermore, they manifested improved protection against secondary pneumococcal pneumonia. Although pulmonary GM-CSF treatment has some drawbacks such as inflammatory activity [211] that can render its use in humans problematic by exacerbating inflammatory lung injury, the results of our study are promising. They suggest that strategies seeking to balance the protective AM replenishment effects and the adverse effect of exuberant inflammation induction via combining an adjunctive anti-inflammatory therapy with GM-CSF treatment may be successful.

Overall, these findings suggest a novel mechanism of influenza-mediated immune suppression that resulted in increased permissiveness of influenza-infected hosts to SBP. Resident AM depletion during influenza infection establishes a niche for secondary pneumococcal infection by altering early cellular innate immunity in the lungs, thereby allowing pneumococcal outgrowth causing lethal pneumococcal pneumonia. The precise functional characterization of this novel finding can change the way researchers look at the alteration of pulmonary cellular innate immunity during sublethal influenza infections. There are likely to be important effects on immunity beyond the bacterial escape studied here. Furthermore, these findings open avenues for novel immunomodulating therapeutic interventions to prevent respiratory bacterial superinfections by quick replenishment of the critical innate immune effectors during both pandemic and seasonal influenza. 


\section{CHAPTER 4. ADJUNCTIVE IMMUNOMODULATOR THERAPY IMPROVES MORTALITY DURING ANTIBIOTIC TREATMENT OF SECONDARY PNEUMOCOCCAL PNEUMONIA}

\section{Introduction}

Globally, pneumonia is the leading killer of children outside of the neonatal period [181]. In addition, there has been little change in mortality due to respiratory tract infections for more than five decades $[119,120]$. SBP is a common complication of influenza, and outcomes are worse in co-infections than in uncomplicated cases [35, 212]. In the 1918 influenza pandemic, the majority of deaths were complicated by SBP, and $S$. pneumoniae was the predominant pathogen [123]. However, the advent of antibiotic therapy in 1940s and the widespread use of effective pneumococcal vaccines in the developed world decreased the frequency of pneumococcal diseases over the last century. Nonetheless, $S$. pneumoniae continues to be the leading bacterial cause of community-acquired pneumonia (CAP) and SBP [213]. For example, during the 2009 H1N1 influenza pandemic, $25 \%$ to $50 \%$ of severe or fatal cases were complicated by SBP, mostly with S. pneumoniae and Staphylococcus aureus [35, 126, 214].

These severe outcomes including mortality are seen in patients with SBP despite appropriate antimicrobial treatment $[212,215]$. Although globally increased rates of antimicrobial resistance among common respiratory bacterial pathogens are a concern $[165,166]$, the mechanism of killing utilized by standard antibiotics also appears to affect treatment outcomes [183, 204]. Ampicillin is considered the first line therapy for bacterial pneumonia in hospitalized children, including those co-infected with influenza [216]. In a murine model of SBP following influenza, treatment with ampicillin was shown to induce robust inflammatory lung injury [202]. This poor treatment outcome, despite its efficient bactericidal activity, was attributed to rapid bacterial cell wall lysis and the release of copious amounts of bacterial PAMPs, including cell wall fragments, potentiating the inflammatory response in the co-infected lungs [204]. Trials in adults have demonstrated an improved cure rate for inpatient CAP treatment with either fluoroquinolone antibiotics or combination therapy with a $\beta$-lactam and a macrolide [217]. Our mouse studies suggest that, along with broadened coverage of atypical pathogens, the addition of the macrolide to the recommended treatment regimen reduces the inflammatory response by decreasing the massive influx of neutrophils and accompanying tissue damage characteristic of SBP [204]. However, treatment with antibiotics alone appears to still be sub-optimal in some patients and in these mouse models.

Due to their potent anti-inflammatory and diverse immunomodulatory activities, corticosteroids have been widely used to treat many inflammatory and immune diseases $[218,219]$. However, the clinical use of corticosteroids as an adjunctive therapy for treating pneumonia has been controversial. Some randomized controlled clinical trials suggest beneficial activity in treating CAP with a significant reduction in the length of hospital stay [220,221] or a decrease in mortality of patients with septic shock [222]. 
However, others have shown no benefit or harmful outcomes [223, 224]. Based on these data and expert opinion, the World Health Organization (WHO) discouraged corticosteroid treatment during the 2009 influenza pandemic [225]. Collectively, there is not currently strong evidence in the literature for recommending adjunctive corticosteroid therapy. Nevertheless, steroids are often used in clinical practice, particularly in severe cases with acute lung injury, suggesting a need for further study [226-228].

We hypothesized that adjunctive corticosteroid therapy would improve the poor outcomes associated with antibiotic treatment of SBP following influenza infection through modulation of inflammatory responses. We tested our hypothesis using a wellestablished murine co-infection model in which we could monitor the progression of SBP in vivo [229]. To analyze the relationship between disease severity and outcomes, we categorized co-infected mice based on the lung bacterial load at the onset of antibiotic treatment to control for its impact on different treatment outcomes. We report here that dexamethasone therapy administered during the inflammatory period of severe SBP has a beneficial effect on outcomes. Early therapy, during primary influenza, is not beneficial and may enhance the infection by interfering with immune responses to the virus.

Additionally, we tested the efficacy of a novel immunomodulator drug, 2,3diacetyloxybenzoic acid (DABA). DABA was designed as a pro-drug of 2,3dihydroxybenzoic acid (DHBA) to increase its lipophilicity and thus increase its intracellular delivery [230]. DHBA is an old drug known by its strong iron chelation activity for a long time [231]. It was also identified as a siderophore secreted from some bacterial pathogens to facilitate iron uptake in the iron limited environment [232]. DHBA was shown to decrease vancomycin-induced nephrotoxicity in rats, which was assumed to be mediated through decreasing free hydroxyl radical formation [233].

However, DHBA showed limited efficacy in treating animal models of acute lung injury and sepsis, whereas its pro-drug, DABA, was shown to attenuate the endotoxininduced acute lung injury in two separate animal models. Early systemic treatment by DABA before and after endotoxin administration could significantly decrease lung microvascular permeability [230]. Therefore, we sought to test if adjunctive DABA therapy would also prevent the increased lung immunopathology associated with antibiotic treatment of SBP. We also tested the efficacy of DABA with early administration during primary influenza infection.

\section{Materials and Methods}

\section{Infectious Agents}

PR8 influenza virus and S. pneumoniae A66.1, a type 3 encapsulated strain, were prepared and used for murine co-infections as described previously (pages 29 and 49). 


\section{Mice}

Six- to 8-week-old female BALB/c mice (Jackson Laboratory, Bar Harbor, ME) were maintained in a Biosafety Level 2 facility in the Animal Resource Center at St. Jude. Animals were given general anesthesia that consisted of $2.5 \%$ inhaled isoflurane (Baxter Healthcare Corporation, Deerfield, IL) prior to all interventions, and all studies were approved by the Animal Care and Use Committee at St. Jude.

\section{Co-infection Model}

Infectious agents were diluted in sterile PBS and administered intranasally in a volume of $100 \mu \mathrm{l}(50 \mu \mathrm{l}$ per nostril) to anesthetized mice held in an upright position. For primary influenza infection, influenza virus was given at a dose of 25 TCID50 per $100 \mu 1$ per mouse, which caused about $10 \%$ weight loss on day 7 p.i (post infection) with no mortality when given alone. To engender SBP, influenza infection was followed on day 7 p.i by bacterial challenge with 200 CFUs of pneumococcus per mouse. Infected mice were weighed and assessed daily for illness and mortality for 7 days after pneumococcal challenge; based on preliminary studies in this model and animal care considerations, any mouse losing more than $26 \%$ of its starting body weight was euthanized and considered to have died on that day.

\section{Imaging and Ampicillin Treatment of Live Co-infected Mice}

Mice were imaged for 60 seconds using an IVIS CCD camera (Caliper Life Sciences, Alameda, CA) daily after pneumococcal challenge to monitor in vivo pneumococcal pneumonia development. Total photon emission from selected and defined areas within the images of each mouse was quantified using Living Image software (Caliper Life Sciences, Alameda, CA) as described previously [152, 203, 204], and expressed as the flux of relative light units per minute. Pneumonia was defined as visible bioluminescence within the thorax and detection of a flux of $>11,000$ relative light units per minute (RLU/min) [229]. Based on previous studies that classified detection stage of SBP into early and late detection $[152,204]$, we assigned mild pneumonia to mice showing thorax bioluminescence and flux of more than 11,000 but less than 90,000 RLU/min, while severe pneumonia was defined for mice with flux of more than 90,000 $\mathrm{RLU} / \mathrm{min}$. Once SBP was detected, ampicillin (Sigma-Aldrich) was given i.p. as 200 $\mathrm{mg} / \mathrm{kg} /$ daily in two divided doses every 12 hours for 5 days.

\section{Flow Cytometric Analysis of Immune Cells in BALF and Post-lavage Lungs}

Following euthanasia by $\mathrm{CO}_{2}$ inhalation, the trachea was exposed and cannulated with a 24-gauge plastic catheter (Becton Dickinson Infusion Therapy Systems, Inc., Sandy, UT). Lungs were lavaged, harvested, and homogenized as described previously (page 30). Then, cell suspensions of BALF and post-lavage lung homogenate were 
centrifuged at $4^{\circ} \mathrm{C}, 350 \times \mathrm{g}$ for $7 \mathrm{~min}$. BALF and lung homogenate supernatants were stored at $-80^{\circ} \mathrm{C}$. Flow cytometry (LSRII, and LSRII Fortessa, Becton Dickinson, San Jose, CA) was performed on the cell pellets after incubation with Fc block (anti-mouse CD16/CD32, BD Bioscience Inc., San Jose, CAio), followed by surface marker staining with cocktail of anti-mouse antibodies conjugated with different fluorescent probes, including CD11c (eFluor 450), F4/80 (FITC), Ly6G (PerCp-Cy5.5), and CD11b (APCeFluor 780; eBioscience Inc., San Diego, CA), or CD3 (FITC), CD4 (APC), and CD8a (eFluor 450 or APC-eFluor 780; eBioscience Inc., San Diego, CA). FlowJo 8.8.6 (Tree Star, Ashland, OR) was used for data analysis, where viable cells were gated from an FSC/SSC plot, and then neutrophils were gated as (CD11 b ${ }^{\text {hi }}{\text { Ly } 6 G^{\text {hi }}}$ CD $11 c^{\text {lo }}$ F4/80 $\left.{ }^{\text {lo }}\right)$. T cells were gated as $\mathrm{CD}^{+} \mathrm{SSC}^{\text {low-int }}$, followed by sub-gating of $\mathrm{CD} 4^{+} \mathrm{CD} 8^{-}$and $\mathrm{CD} 8^{+}$ $\mathrm{CD}^{-} \mathrm{T}$ cells subsets. Total viable cells were counted and absolute numbers of different cell types were calculated as described previously (page 30).

\section{Dexamethasone Treatment Regimen}

Dexamethasone sodium phosphate injection solution $(4 \mathrm{mg} / \mathrm{ml}$; APP

Pharmaceuticals, Schaumburg, IL) was diluted by sterile PBS solution to $0.5 \mathrm{mg} / \mathrm{ml}$. In combined treatment experiments, dexamethasone dose $(2.5 \mathrm{mg} / \mathrm{kg} /$ day $)$ or vehicle (PBS) was given i.p. once daily 2-3 hours after ampicillin injection for 5 days as adjunctive therapy. In early treatment experiments, dexamethasone or PBS was given i.p. once daily starting from day 3 until day 13 after influenza infection.

\section{2,3-Diacetyloxybenzoic Acid Treatment Regimen}

DABA was a kind gift from Dr. Ze-Qi Xu (Advanced Life Sciences Co., Woodridge, IL). DABA injection solution $(25 \mathrm{mg} / \mathrm{ml})$ was prepared freshly according to the manufacturer's instructions. Briefly, $50 \mathrm{mg}$ of the drug was dissolved in $0.5 \mathrm{ml}$ of $8.4 \%$ sodium bicarbonate injection, USP. Then, $1.5 \mathrm{ml}$ of sterile distilled water was added followed by vortex and sonication until the drug powder gets completely dissolved, then the solution was filter sterilized. The injection solution was kept at $4{ }^{\circ} \mathrm{C}$ and was used within 1-2 days. Mice were i.p injected with DABA in the same dose as used before for treating acute lung injury $(150 \mathrm{mg} / \mathrm{kg} /$ day $)$ [230], but following the same regimen as dexamethasone.

\section{Measurement of Viral Titers}

Influenza viral titers were measured in the stored post-lavage lung homogenate supernatants by TCID50 assay in MDCK cells. 


\section{Measurement of Total Protein and Albumin Levels in BALF Supernatant}

BALF supernatant aliquots were thawed and total proteins levels were measured spectrophotometrically using a NanoDrop 2000 Spectrophotometer (Thermo Fisher Scientific, Wilmington, DE). To measure albumin levels, BALF supernatant samples were diluted in sterile PBS, then albumin concentrations were measured using an ELISA kit (USCN Life Science Inc, Houston, TX).

\section{Statistical Analysis}

Comparison of survival between groups of mice was done with the log-rank chisquared test on the Kaplan-Meier survival data. Means of relative bioluminescence units per minute were compared between groups using analysis of variance (ANOVA). Comparison of weight loss, immune cells numbers, or viral titers between groups of mice was done using Mann-Whitney $U$ test for pair-wise comparisons. A p-value of $<0.05$ was considered significant for these comparisons. Prism 4 for Windows (GraphPad Software, Inc., V 4.03) was used for all statistical analyses.

\section{Results}

\section{Ampicillin Treatment during Secondary Pneumococcal Pneumonia Fails to Rescue Mice Despite Viral and Bacterial Clearance}

To engender SBP, naïve BALB/c mice were intranasally infected by a sublethal dose of influenza virus, followed by a small inoculum of pneumococcus on day 7 after influenza infection (Figure 4-1A). Within $48 \mathrm{~h}$ after bacterial inoculation, co-infected mice developed SBP that could be detected in vivo by bioluminescence imaging. Without treatment all co-infected mice succumbed to bacterial pneumonia within a few days (Figure 4-1B). To confirm the poor efficacy of a standard antibiotic treatment in rescuing mice with SBP, I started ampicillin treatment upon pneumonia detection via bioluminescence (Figure 4-1A and C). As seen previously [202, 204, 234], ampicillin could only rescue about $40 \%$ of pneumonic mice (Figure 1B), despite rapid declines in bacterial burden within $12 \mathrm{~h}$ after the first dose of ampicillin (Figure 4-1C and D). Viral titers were undetectable in the alveolar airspaces $24 \mathrm{~h}$ after the first ampicillin dose (data not shown).

\section{Differential Outcomes Depend on the Severity of Secondary Pneumococcal Pneumonia at the Onset of Antibiotic Treatment}

To determine if the severity of SBP at the onset of antibiotic treatment affects treatment outcomes, we classified the severity of pneumonic mice prior to antibiotic treatment into mild or severe pneumonia. This classification was based on the 

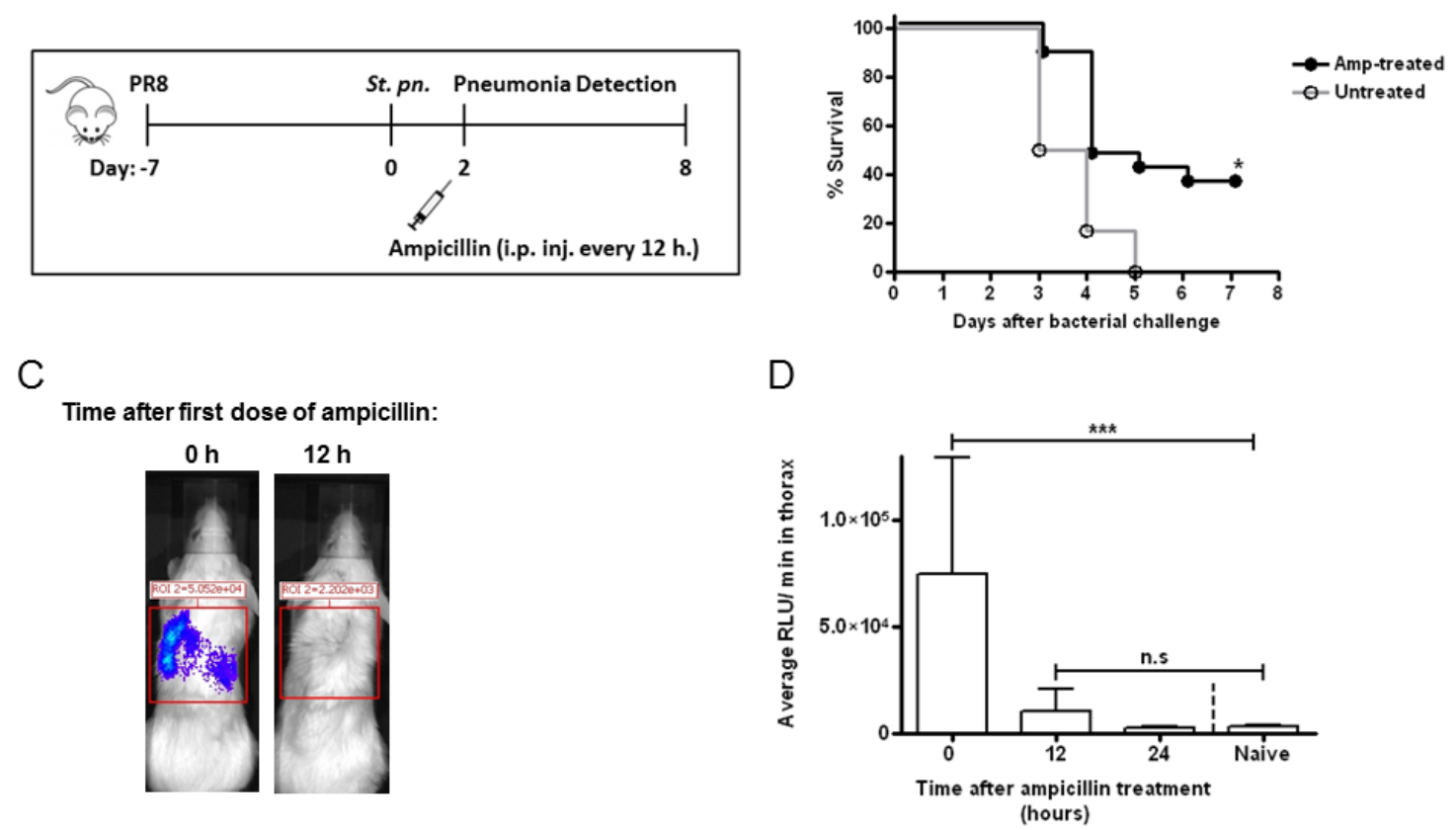

Figure 4-1. Ampicillin treatment of secondary pneumococcal pneumonia

(A) Diagram showing the co-infection model where mice are intranasally infected by influenza virus (PR8) followed by secondary pneumococcal challenge (S. pneumoniae) 7 days later. The ampicillin treatment regimen is started upon secondary pneumococcal pneumonia detection (often on day 9 p.i). (B) Survival rates of co-infected mice either untreated $(n=6)$ or after ampicillin treatment $(n=17)$. (C) Two representative bioluminescent images of co-infected mice showing development of secondary pneumococcal pneumonia $(0 \mathrm{~h})$, and effective pneumococcal killing $12 \mathrm{~h}$ after first ampicillin dose. (D) Significant reduction of throax bioluminescence flux from mice with secondary pneumococcal pneumonia $(n=8)$ after first doses of ampicillin treatment to levels comparable to naïve mice $(n=6$, measurements expressed as relative luminescence units (RLU)/min).

${ }^{*} P<0.05$ by log-rank test on the Kaplan-Meier survival data, compared with untreated group (Panel B). $* * * P<0.001$ by ANOVA test, compared with all other groups (Panel $\mathrm{D})$. The bar graph shows the average $\pm \mathrm{SD}$. 
pneumococcal load in co-infected lungs, as monitored through bioluminescence imaging as defined in the methods (Figure 4-2A).

Ampicillin treatment was associated with disparate mortality outcomes in mice with different degrees of pneumonia at the onset of treatment (Figure 4-2B). All ampicillin-treated mice with severe pneumonia succumbed to pneumonia in a similar pattern to that of the untreated group. In contrast, more than $60 \%$ of ampicillin-treated mice with mild pneumonia were rescued (Figure 4-2B). These differential mortality outcomes after ampicillin treatment were not due to lack of ampicillin bactericidal activity in mice with severe pneumonia; the first ampicillin dose demonstrated in vivo efficacy with rapid bacterial killing, reflected by the significant decrease in bioluminescence signals to levels comparable to uninfected mice, in a similar pattern to ampicillin-treated mice with mild pneumonia (Figure 4-2C).

However, differential accumulation of neutrophils was observed within the alveolar airspaces of mildly and severely pneumonic mice $26 \mathrm{~h}$ after the first ampicillin dose (Figure 4-3A). Interestingly, ampicillin treatment of mice with severe SBP induced significantly greater accumulation of neutrophils than in the untreated mice with SBP at the same time point (day 10 after influenza infection) (Figure 4-3A). Furthermore, significantly higher total proteins levels were detected in the BALF supernatant of ampicillin-treated mice with severe pneumonia (Figure 4-3B).

\section{Adjunctive Dexamethasone Treatment Rescues Ampicillin-treated Mice with Severe Secondary Pneumococcal Pneumonia}

Since treatment by ampicillin alone failed to rescue mice with severe pneumonia, despite its efficient bactericidal activity, we hypothesized that an anti-inflammatory agent could dampen the associated inflammatory responses and improve outcomes. Adjunctive dexamethasone therapy significantly improved the survival rate, rescuing about $70 \%$ of mice with severe pneumonia (Figure 4-4A). Mice with mild pneumonia also demonstrated a modest increase in survival that was not statistically significant after adjunctive dexamethasone treatment, but suffered morbidity reflected by significant delay in regaining their body weight as compared to the PBS and ampicillin-treated control group (Figure 4-4B). Improved outcomes with combined therapy were associated with significantly decreased neutrophil accumulation after the second dose (Figure 4-5A) and significantly reduced serum albumin leakage into alveolar airspaces after the first dose (Figure 4-5B).

\section{Early Dexamethasone Treatment Leads to Loss of Its Protective Activity}

To test if dexamethasone administration early after influenza infection can enhance its protective activity and increase survival of ampicillin-treated pneumonic mice, we started dexamethasone or PBS treatment 3 days after influenza infection and continued thereafter until day 13 p.i using the same co-infection model. As before, 
A

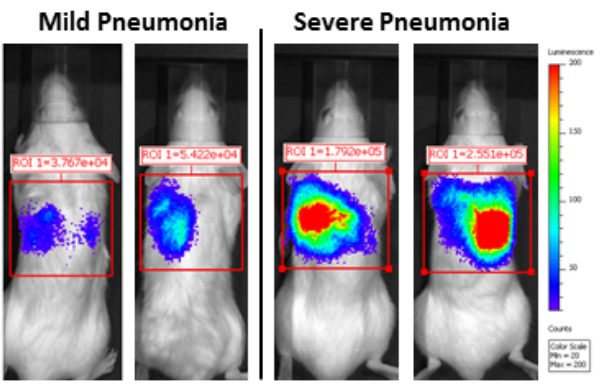

C

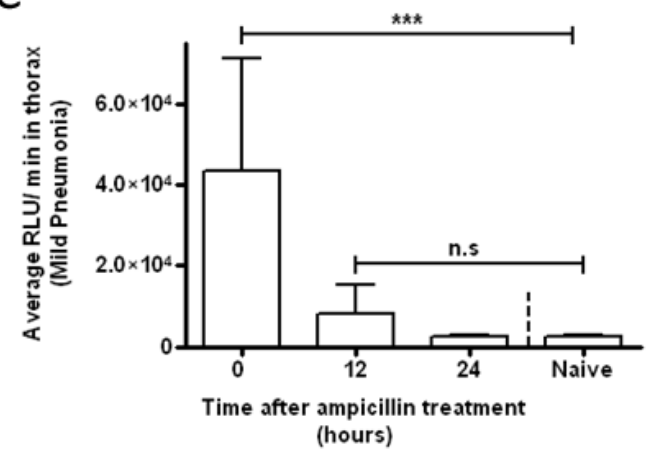

B
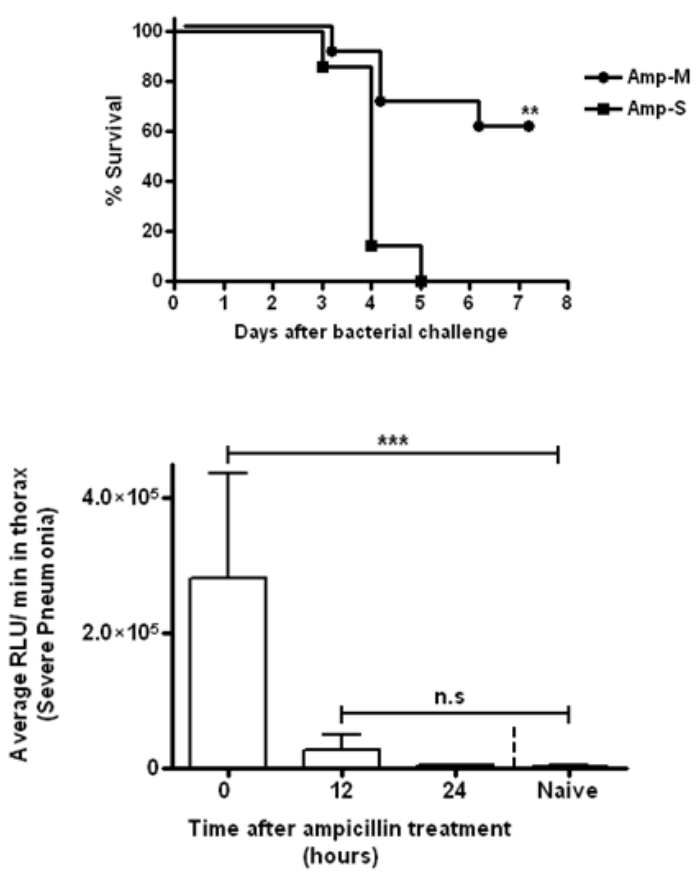

Figure 4-2. Differential mortality outcomes depend on the severity of secondary pneumococcal pneumonia at the onset of antibiotic treatment

(A) Representative images showing bioluminescence measurements from two different co-infected mice with mild or severe secondary pneumococcal pneumonia. Images were taken $48 \mathrm{~h}$ after secondary pneumococcal challenge and prior to ampicillin treatment. (B) Survival rates of co-infected mice with mild (Amp-M, $n=10)$ or severe (Amp-S, $n=7$ ) secondary pneumococcal pneumonia treated with ampicillin. (C) Significant reduction in thorax bioluminescence flux from co-infected mice with either mild or severe secondary pneumococcal pneumonia after first doses of ampicillin treatment $(n=10)$ to levels comparable to naïve mice $(\mathrm{n}=6)$.

** $P<0.01$ by log-rank test on the Kaplan-Meier survival data, compared with Amp-S group (Panel B), *** $P<0.001$ by ANOVA test, compared with all other groups (Panel $\mathrm{C})$. The bar graphs show the average $\pm \mathrm{SD}$. 
A

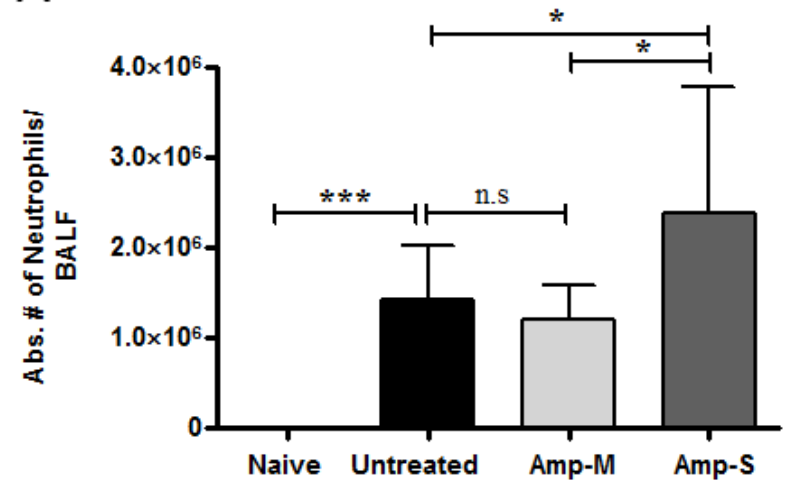

B

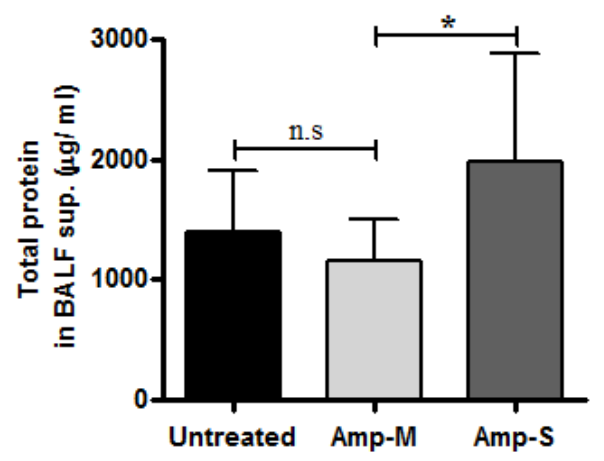

Figure 4-3. Differential lung immunopathology depends on the severity of secondary pneumococcal pneumonia at the onset of antibiotic treatment

(A) Absolute numbers of neutrophils in BALF of mock-infected (naïve, white-filled, $\mathrm{n}=$ 12), untreated co-infected mice on day 10 after influenza infection (black-filled, $n=15$ ), or ampicillin-treated mice with mild (light gray-filled, $n=7$ ) or severe (dark gray-filled, $n$ $=6$ ) secondary pneumococcal pneumonia 3 days after bacterial challenge (i.e. $26 \mathrm{~h}$ after first ampicillin dose). (B) Total proteins levels in BALF supernatant of untreated coinfected mice on day 10 after influenza infection (black-filled, $n=5$ ), ampicillin-treated mice with mild (light gray-filled, $n=7$ ) or severe (dark gray-filled, $n=9$ ) pneumonia, measured $26 \mathrm{~h}$ after first ampicillin dose.

$* P<0.05, * * * P<0.001$ by ANOVA test, compared with all other groups (Panel A and $\mathrm{B})$. The bar graphs show the average $\pm \mathrm{SD}$. 
A
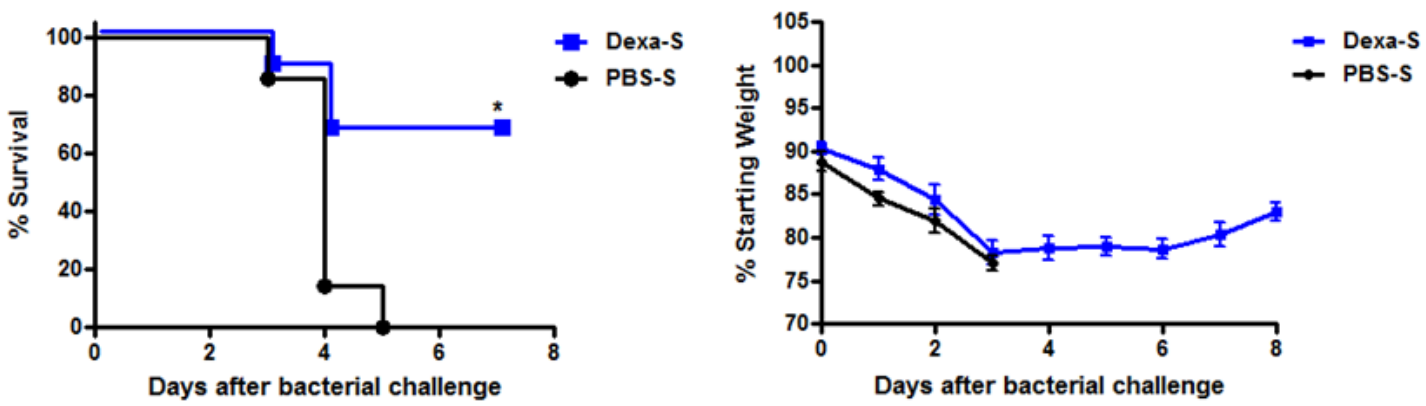

B
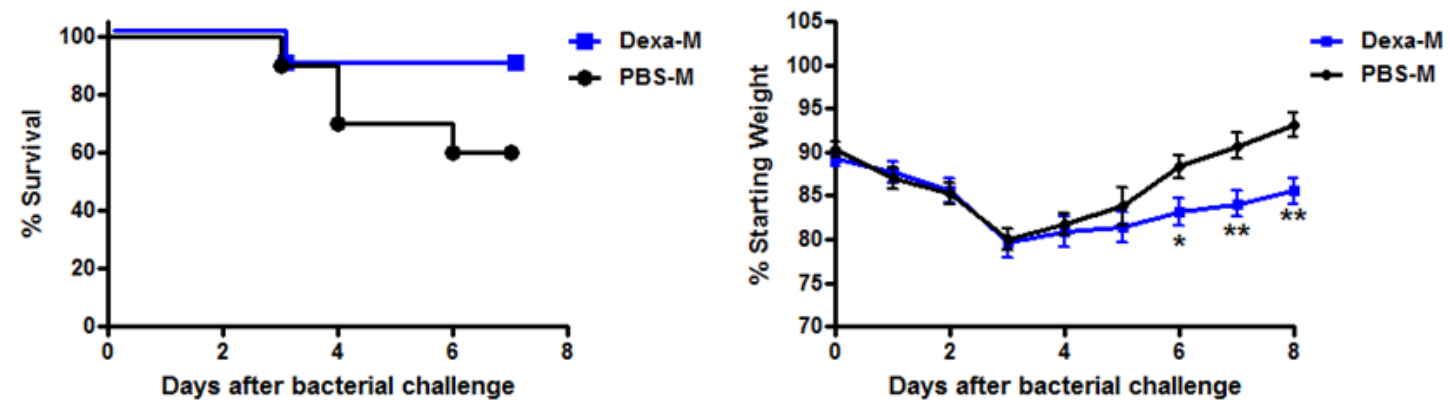

Figure 4-4. Adjunctive dexamethasone treatment rescues ampicillin-treated mice with severe secondary pneumococcal pneumonia

Survival rates and body weight loss curves of ampicillin-treated mice with either severe (Panel $A, n \geq 7$ ) or mild (Panel $B, n \geq 9$ ) secondary pneumococcal pneumonia with adjunctive dexamethasone or mock (PBS) therapy.

${ }^{*} P<0.05$, ${ }^{*} P<0.01$ compared with adjunctive PBS therapy group by log-rank test on the Kaplan-Meier survival data (Panel A), or Mann-Whitney U test for body weight loss curve (Panel B). Data are expressed as the average \pm SEM. 
A

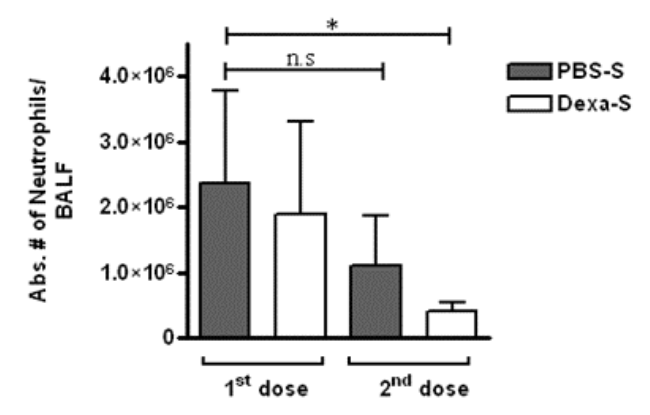

$\mathrm{B}$

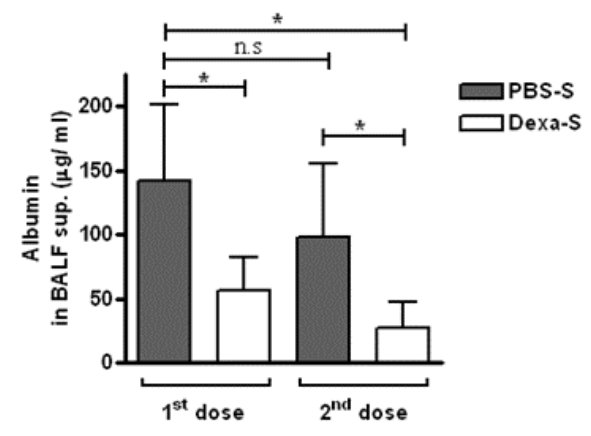

Figure 4-5. Adjunctive dexamethasone therapy improves lung inflammation and pulmonary vascular permeability in mice with severe secondary pneumococcal pneumonia

(A) Absolute numbers of neutrophils within alveolar airspaces of mice with severe secondary pneumococcal pneumonia, measured $26 \mathrm{~h}$ after the first or second dose of dexamethasone (white-filled, $n=4$ or 3 , respectively) or mock therapy (dark gray-filled, $\mathrm{n}=6$ or 5 , respectively) combined with ampicillin treatment. (B) Albumin levels in BALF supernatant harvested from mice with severe secondary pneumococcal pneumonia $26 \mathrm{~h}$ after the first or second dose of adjunctive dexamethasone (white-filled, $\mathrm{n}=4$ or 3 , respectively) or mock therapy (dark gray-filled, $n=6$ at both time points) combined with ampicillin treatment.

${ }^{*} P<0.05$ by Mann-Whitney $\mathrm{U}$ test. The bar graphs show the average $\pm \mathrm{SD}$. 
ampicillin treatment was initiated upon detection of SBP. Interestingly, early dexamethasone treatment did not augment the survival improvement of ampicillin-treated pneumonic mice; rather, it led to loss of its protective activity (Figure 4-6A). Survival of ampicillin-treated mice with severe pneumonia dropped from about $70 \%$ with adjunctive dexamethasone therapy (Figure 4-4A) to about 30\% with early dexamethasone regimen (Figure 4-6A). Furthermore, ampicillin-treated mice with mild pneumonia demonstrated a modest, non-significant increase in mortality after early dexamethasone treatment as compared to mock-treated group (Figure 4-6A). In addition, similar to the poor morbidity outcome of adjunctive therapy in mice with mild pneumonia, early dexamethasone treatment was associated with increased body weight loss from primary influenza and delayed body weight regain after ampicillin treatment of SBP (Figure 4-6B).

\section{Early Dexamethasone Treatment Suppresses Adaptive Immunity}

Glucocorticoids are known to suppress adaptive immunity [235]. Therefore, we hypothesized that the increased morbidity associated with early dexamethasone therapy during primary influenza infection was due to suppressed adaptive immunity, leading to elevated influenza viral titers and increased lung injury. We measured the numbers of T cells in both BALF and post-lavage lungs on day 7 after influenza infection under early dexamethasone or mock treatment (i.e., $24 \mathrm{~h}$ after the fourth dose of dexamethasone or PBS). As expected, early dexamethasone treatment significantly reduced the absolute numbers of $\mathrm{CD}^{+}$and $\mathrm{CD} 8^{+} \mathrm{T}$ cells in lungs as compared to mock treatment (Figure 4-7A). To determine if this reduction in the respiratory pool of $\mathrm{T}$-cells was associated with impaired influenza viral clearance, we measured influenza viral titers on day 7 p.i. Early dexamethasone-treated mice had significantly higher influenza viral titers than mock-treated mice (Figure 4-7B).

\section{Adjunctive DABA Treatment Improves Survival of Ampicillin-treated Mice with Secondary Pneumococcal Pneumonia}

The findings that adjunctive dexamethasone therapy worsened the morbidity of ampicillin-treated mice with mild SBP, reflected by the delayed body weight regain (Figure 4-4B), put the proposed use of dexamethasone into question. Therefore, we tested the efficacy of a novel immunomodulator drug, DABA, for treating SBP using the same treatment regimen as dexamethasone. We found that adjunctive DABA therapy improved survival of ampicillin-treated mice with severe or mild SBP in a rate similar to that of adjunctive dexamethasone treatment. Combined DABA plus ampicillin treatment could significantly increase the survival rate of mice with severe SBP from $0 \%$ to about $70 \%$. In addition, adjunctive DABA therapy could rescue all ampicillin-treated mice with mild SBP, but the increase in the survival rate was not statistically significant compared to the mice group subjected to mock adjunctive therapy (Figure 4-8A and $\mathbf{B}$ ).

In contrast to dexamethasone treatment, adjunctive DABA therapy did not worsen 
A
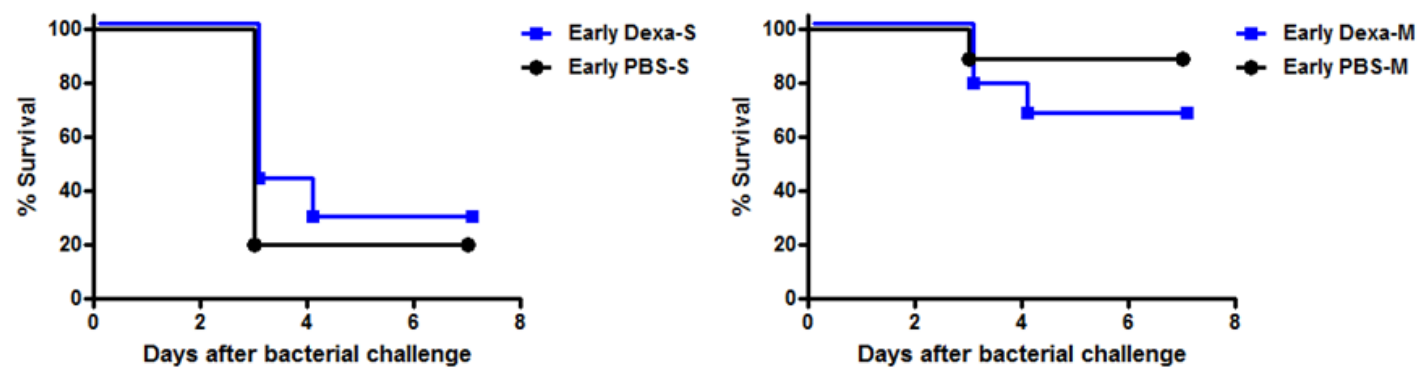

B

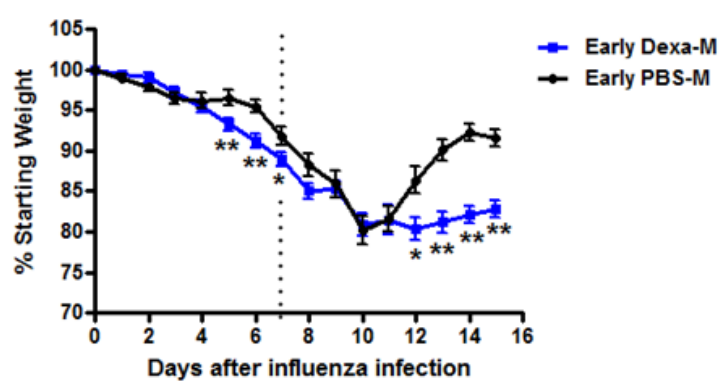

Figure 4-6. Early dexamethasone treatment leads to loss of its protective activity

(A) Survival rates of co-infected mice with early dexamethasone or mock (PBS) therapy started during primary influenza infection, and continued with ampicillin treatment after detection of severe (Early Dexa-S, $n=7$ or Early PBS-S, $n=5$ respectively) or mild (Early Dexa-M, $\mathrm{n}=9$ or Early PBS-M, $\mathrm{n}=9$, respectively) secondary pneumococcal pneumonia. (B) Body weight loss curve of influenza-infected mice with early dexamethasone or mock (PBS) therapy, challenged with bacteria on day 7 p.i, and treated later with ampicillin after detection of mild secondary pneumococcal pneumonia $(\mathrm{n}=9)$. ${ }^{*} P<0.05,{ }^{*} P<0.01$ by Mann-Whitney $\mathrm{U}$ test (Panel B). Data are expressed as the average \pm SEM. 

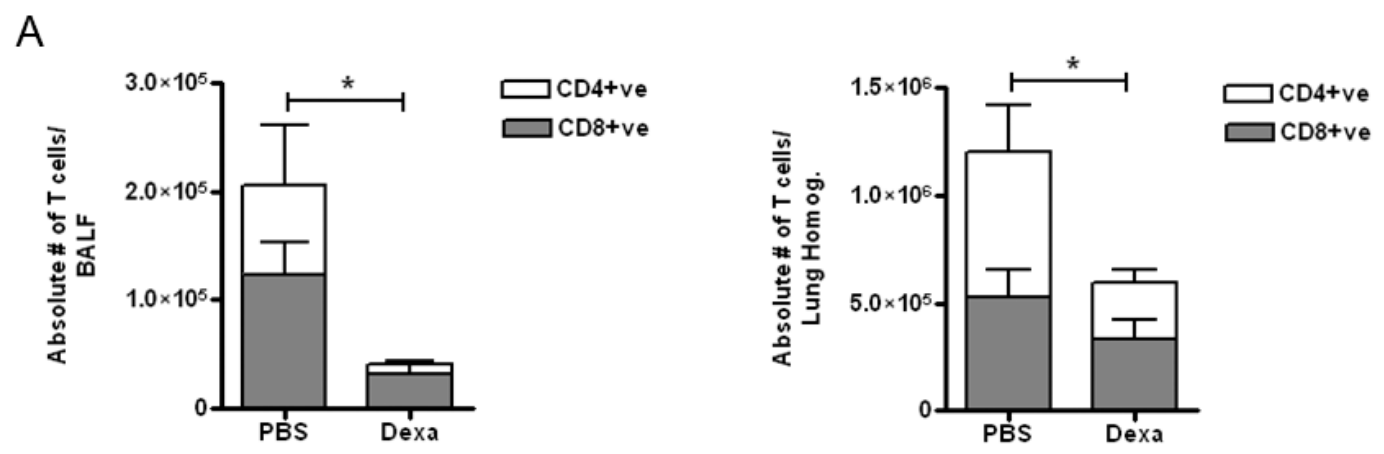

$\mathrm{B}$

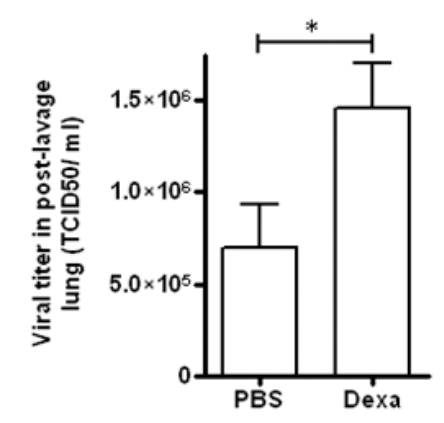

Figure 4-7. Early dexamethasone treatment suppresses adaptive immunity

(A) Absolute numbers of $\mathrm{CD}^{+}$and $\mathrm{CD}^{+} \mathrm{T}$ cells within alveolar airspaces (BALF), or in post-lavage lungs of influenza-infected mice measured on day 7 p.i with early dexamethasone or mock (PBS) therapy ( $n=4$ or 5 , respectively). (B) Influenza viral titers in post-lavage lungs of influenza-infected mice measured on day 7 p.i with early dexamethasone or mock (PBS) therapy ( $\mathrm{n}=4$ or 5 , respectively).

${ }^{*} P<0.05$ by Mann-Whitney $U$ test. The bar graphs show the average $\pm \mathrm{SD}$. 
A
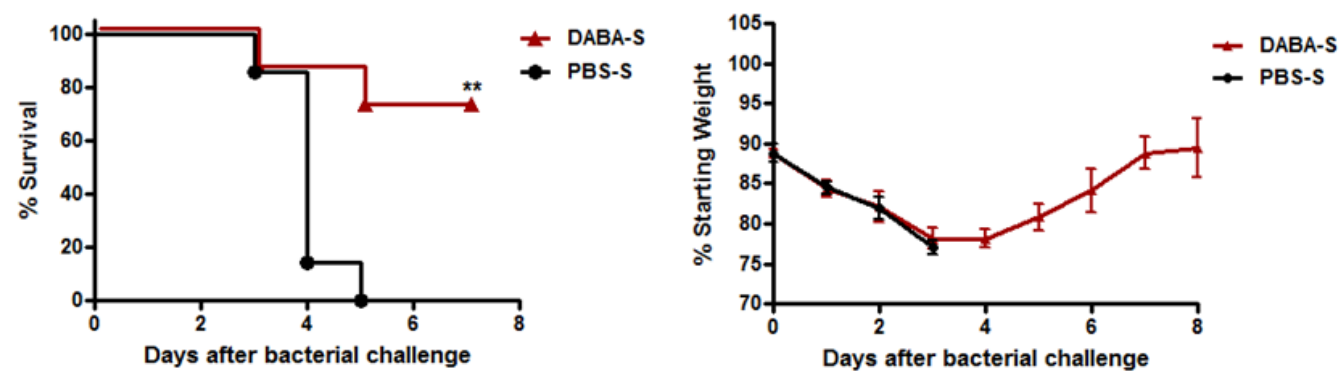

B
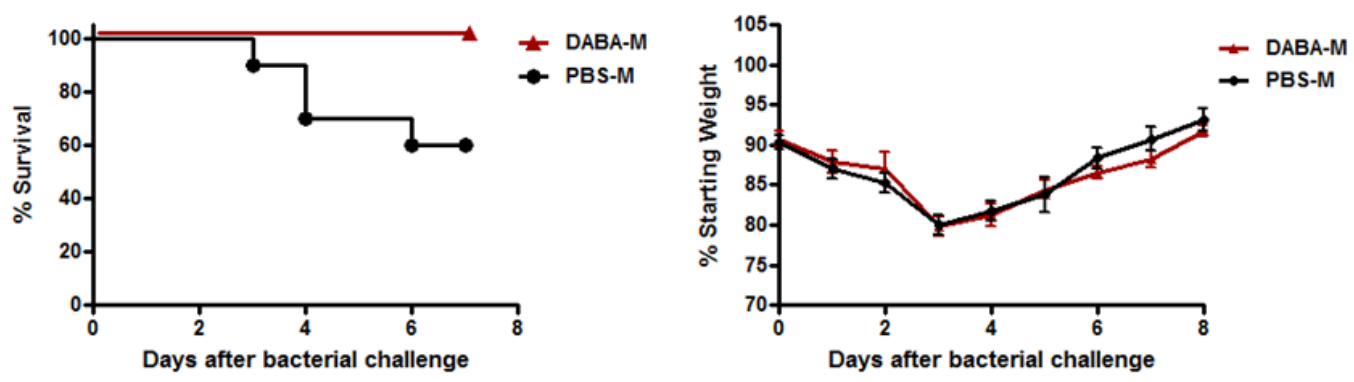

C

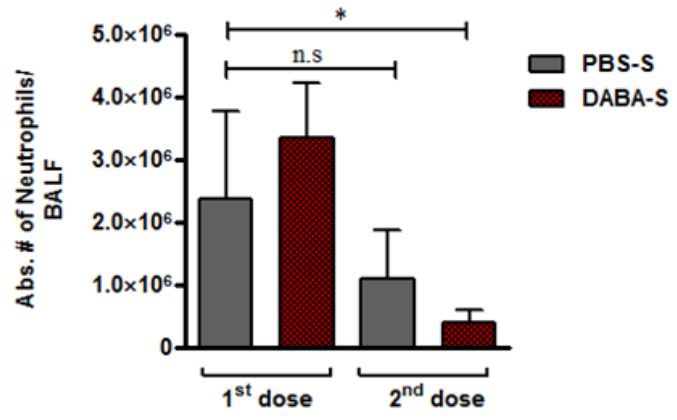

Figure 4-8. Adjunctive 2,3-diacetyloxybenzoic acid treatment improves survival of ampicillin-treated mice with secondary pneumococcal pneumonia

Survival rates and body weight loss curves of ampicillin-treated mice with either severe (Panel $\mathrm{A}, \mathrm{n}=7$ for each group) or mild (Panel $\mathrm{B}, \mathrm{n} \geq 8$ for each group) secondary pneumococcal pneumonia with adjunctive 2,3-diacetyloxybenzoic acid (DABA) or mock (PBS) $^{*}$ therapy. (C) Absolute numbers of neutrophils within alveolar airspaces of mice with severe secondary pneumococcal pneumonia, measured $26 \mathrm{~h}$ after the first or second dose of adjunctive DABA (red-filled, $\mathrm{n}=5$ or 3 , respectively) or mock therapy (dark gray-filled, $\mathrm{n}=6$ or 5 , respectively) combined with ampicillin treatment.

** $P<0.01$ by log-rank test on the Kaplan-Meier survival data, ${ }^{*} P<0.05$ by MannWhitney $U$ test (Panel C). Data are expressed as the average \pm SEM (Panel A and B), or as the average $\pm \mathrm{SD}$ (Panel $\mathrm{C})$.

"I used the same mock-treated groups in (Figure 4-4), as I used different drug treatment in the same experimental setting. 
the morbidity of ampicillin-treated mice with mild SBP. This was implicated by monitoring the body weight recovery of mice with mild SBP, as DABA-treated mice showed comparable rate of body weight regain to the mice group with adjunctive mock therapy (Figure 4-8A and B). Improved survival with combined DABA therapy of mice with severe SBP was accompanied by significantly decreased neutrophil accumulation after the second dose (Figure 4-8C).

\section{Early DABA Treatment Does Not Worsen the Morbidity of Influenza-infected Mice}

Another caveat of dexamethasone use that was revealed in our model of SBP is the significant impact of changing its administration timing. We demonstrated that early dexamethasone therapy during the primary influenza infection had a negative impact on the course of influenza infection and was associated with increased morbidity of influenza-infected mice. Therefore, we sought to test if early DABA treatment would have similar bad outcomes as early dexamethasone therapy. Early DABA treatment was initiated from day 3 until day 13 after influenza infection, while ampicillin treatment was given upon detecting SBP. Early DABA treatment did not worsen the morbidity of influenza-infected mice (Figure 4-9B). Nonetheless, early DABA therapy showed partial loss of their protective activity against mortality of ampicillin-treated mice with severe SBP (Figure 4-9A), in a pattern similar to that of early dexamethasone therapy.

\section{Discussion}

Historically, death from SBP was a common feature of all influenza pandemics in the pre-antibiotic era [123]. Indeed, mortality from pneumococcal pneumonia significantly decreased after the discovery of penicillin [236]. Nevertheless, SBP still contributes significantly to morbidity and mortality during seasonal influenza and recent influenza pandemics, despite the use of effective antibiotics [214]. Furthermore, rates of mortality due to pneumococcal pneumonia have been relatively stable throughout the antibiotic era even with the routine use of more advanced $\beta$-lactam antibiotics [237, 238]. In a similar pattern, ampicillin treatment of SBP in our murine model demonstrated therapeutic failure here and in previous studies [202, 204, 234]. Despite its effective rapid bactericidal activity and significant pneumococcal clearance, ampicillin therapy failed to rescue more than 50\% of mice with SBP. This has been previously attributed to rapid bacterial cell wall lysis, accompanied by release of bacterial cell wall fragments activating exuberant inflammatory responses in lungs, thereby leading to increased morbidity and mortality [204].

As the degree of severity of SBP may affect ampicillin treatment outcomes, we determined pneumococcal load in vivo using a sensitive non-invasive bioluminescence imaging to approximate pneumococcal outgrowth in the co-infected lungs prior to treatment, then classified SBP into mild or severe based on this surrogate for pneumococcal load. Interestingly, ampicillin treatment caused disparate mortality rates in mice with different degrees of pre-treatment severity of SBP. This finding suggests 

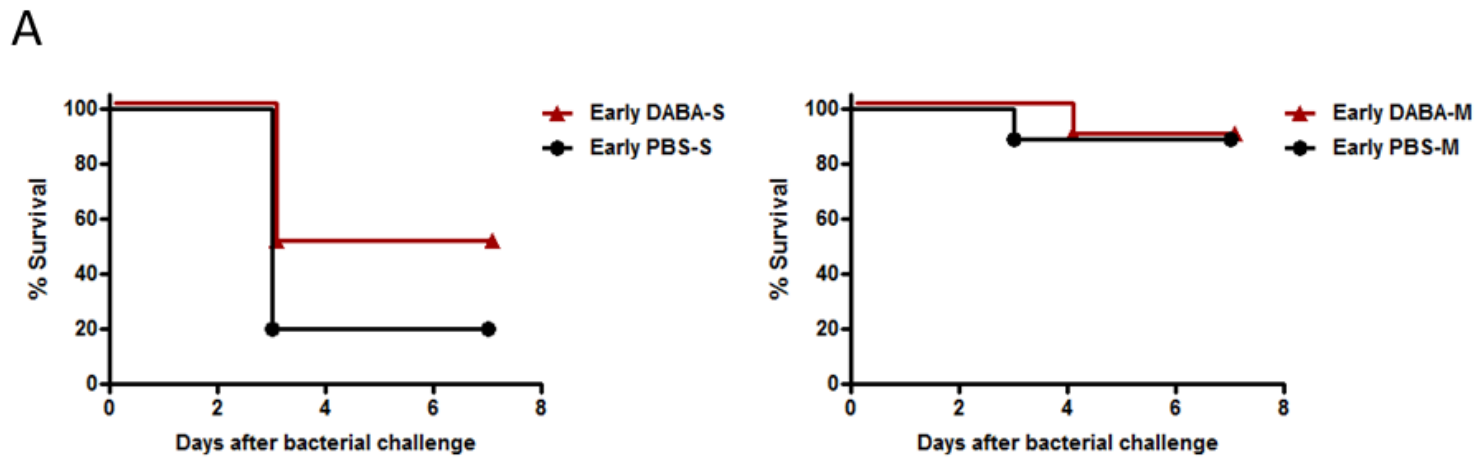

B

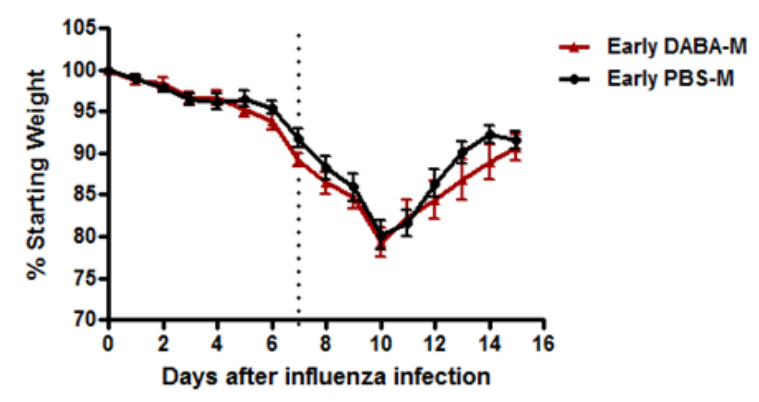

Figure 4-9. Early 2,3-diacetyloxybenzoic acid treatment does not worsen morbidity of influenza-infected mice

(A) Survival rates of co-infected mice with early 2,3-diacetyloxybenzoic acid (DABA) or mock (PBS) ${ }^{*}$ therapy started during primary influenza infection, and continued with ampicillin treatment after detection of severe (Early DABA-S, $n=4$ or Early PBS-S, $n=$ 5 ) or mild ( $\mathrm{n}=9$ for each group) secondary pneumococcal pneumonia. (B) Body weight loss curve of influenza-infected mice with early DABA or mock therapy, challenged with bacteria on day 7 p.i, and treated later with ampicillin after detection of mild secondary pneumococcal pneumonia $(n=9)$. Data are expressed as the average \pm SEM.

*I used the same mock-treated groups in (Figure 4-6), as I used different drug treatment in the same experimental setting. 
a direct correlation between pneumococcal load size at the onset of antibiotic therapy and the poor survival outcomes during antibiotic treatment of SBP. This relationship was inferred through modulating the degree of lung immunopathology induced after bacterial cell lysis with ampicillin. Thus, ampicillin treatment of mice with severe SBP aggravated lung immunopathology compared to those with mild SBP. Yet, both groups of mice showed comparable rapid pneumococcal clearance after the first two doses of ampicillin. The differential ampicillin treatment-associated immunopathology was evident on the second day of treatment, when mice with severe SBP had significantly increased accumulation of neutrophils within the alveolar airspaces, and increased pulmonary vascular permeability compared to ampicillin-treated mice with mild SBP.

To protect against the lung immunopathology induced during antibiotic treatment of SBP, we used the standard anti-inflammatory drug dexamethasone, which is well known for its potent inflammatory suppressive and immunomodulatory activities. Dexamethasone is one of the synthetic corticosteroids that are clinically used to treat several inflammatory disorders and autoimmune diseases [219]. However, the benefits of adjunctive corticosteroid therapy of CAP have been under debate for a long time [227, 239] due to conflicting results from different studies of this potential therapy [220, 221, $223,224,240-242]$. In our murine model of SBP, adjunctive dexamethasone treatment improved survival rates of pneumonic mice. Interestingly, best results were observed in ampicillin-treated mice with severe SBP, in which treatment-associated immunopathology was significantly attenuated. This was achieved after the second dose of dexamethasone, where accumulation of neutrophils within the alveolar airspaces was significantly reduced compared to mock plus ampicillin-treated mice. Furthermore, a significant decrease in pulmonary vascular permeability was observed one day after the first dose of dexamethasone. These treatment outcomes of severe SBP in our murine model are consistent with several randomized controlled trials that showed that the beneficial outcomes of adjunctive corticosteroids therapy are limited to severe cases of community-acquired pneumonia in humans [220, 221, 241, 242]. We used dexamethasone at a dose $(2.5 \mathrm{mg} / \mathrm{kg})$ that was previously shown to suppress the pulmonary inflammatory responses in various murine models of airway inflammation, such as endotoxin-induced acute lung injury, house dust-induced asthma, and allergic bronchopulmonary aspergillosis [243-245]. Other researchers have shown that dexamethasone treatment from days 3-14 after highly pathogenic avian influenza infection had no beneficial effect on acute respiratory distress syndrome caused by the $\mathrm{H} 5 \mathrm{~N} 1$ infection in mice [246], data which support our findings regarding the poor outcomes associated with early dexamethasone treatment during primary influenza infection. Additionally, our dose of dexamethasone is clinically relevant to the corticosteroid doses, which showed beneficial outcomes as adjunctive therapy of adult humans with severe CAP [247-249].

However, corticosteroids have potential drawbacks, as they can cause systemic immunosuppression $[219,235]$. Therefore, we tested the effect of early administration of dexamethasone during primary influenza infection to determine whether it would remain beneficial in ampicillin-treated mice with severe SBP. As expected, early dexamethasone treatment significantly decreased numbers of both $\mathrm{CD}^{+}$and $\mathrm{CD} 4{ }^{+} \mathrm{T}$ cells in the lungs on 
day 7 after influenza infection. This was associated with delayed influenza viral clearance and accelerated morbidity progression during primary influenza infection and before secondary pneumococcal challenge. This had a negative impact on ampicillin treatmentassociated fatality with eventual loss of the protective functions of the adjunctive dexamethasone regimen in mice with either mild or severe SBP.

Therefore, we tested the efficacy of another novel immunomodulator drug DABA that could effectively attenuate the endotoxin-induced acute lung injury in 2 different animal models by decreasing the pulmonary vascular permeability. Adjunctive DABA therapy improved survival of ampicillin-treated mice with SBP, with more pronounced effect on mice with severe SBP associated with significant decrease in neutrophil accumulation within the alveolar airspaces. Additionally, DABA did not worsen the morbidity of mice with mild SBP. Furthermore, early DABA therapy during influenza infection did not have detrimental effect on the morbidity of influenza-infected mice. Nevertheless, early DABA therapy did not augment the improved survival of adjunctive DABA-treated mice, but rather it led to a partial loss of its protective functions.

Taken together, our data suggest that the general unrestricted use of adjunctive corticosteroid therapy during influenza or CAP is likely not warranted. Several factors may participate in balancing the beneficial and detrimental outcomes after systemic corticosteroid treatment, such as the drug dose, the onset and duration of treatment, the causative infectious agents, and whether the pneumonia is caused by a single bacterial or viral agent or is due to a co-infection. Our findings demonstrate that the bacterial burden during pneumonia has an impact on treatment outcomes with larger burdens generating more inflammation and higher mortality upon antibiotic-mediated lysis. Furthermore, these experiments support the observed preferential beneficial activity of corticosteroids when combined with antibiotic treatment in severe cases of CAP. Interestingly, our data point out a significant impact of the timing of dexamethasone therapy on its protective functions, which would be of great importance in modifying treatment protocols of severe cases of SBP following viral infections. Thus, our experiments suggest that early corticosteroid administration during primary influenza infection can worsen adaptive immunity against influenza infection, thereby increasing viral titers and consequently increasing viral-mediated lung damage. Late treatment with steroids, when virus has cleared and inflammation is driving disease, can improve outcomes. Additionally, our preliminary experiments on adjunctive DABA therapy showed promising results for treating CAP without detrimental impact on mild cases. However, it would be interesting to investigate the mechanism of action of DABA treatment in the context of antibiotic therapy of SBP, which may promote its clinical use during treatment of CAP. Finally, our study further advances an animal model with sophisticated tools for classifying the severity of bacterial pneumonia, which will help investigating the efficacy of different immunomodulators as adjunctive therapy, as well as different or new classes of antibiotics for treatment of SBP. 


\section{CHAPTER 5. GENERAL DISCUSSION, INSIGHTS, AND FUTURE DIRECTIONS}

Bacterial super-infections have been implicated to mediate the increased morbidity and deaths during influenza pandemics, epidemics, and even seasonal influenza, with consequent tremendous socioeconomic burden [36, 120]. Their great clinical impact was initially observed long ago, when a French physician, René Laennec, observed the increased frequency of "double" pneumonia as a fatal complication during an influenza epidemic in 1803 [121]. Later, during the 1918 influenza pandemic, many investigators also pointed out the significant contribution of SBP in causing the most deaths in an event that exceeded 30 million fatal cases worldwide. Even though the discovery of penicillin and other antibiotics led to a significant reduction in the mortality rate due to SBP, bacterial super-infections remained a serious medical problem during influenza outbreaks and pandemics, such as the 1957, 1968, and 2009 influenza pandemics [35].

S. pneumoniae is the predominant secondary bacterial pathogen during influenza infections. Because of its nasopharyngeal colonization in some asymptomatic carriers, pneumococcus can easily spread among influenza-infected patients via respiratory droplet inhalation. However, unique lethal synergism has been observed between primary influenza infections and secondary pneumococcal infections.

Although many investigators have established the respiratory viral-bacterial synergism in animal models, the mechanisms underlying it are still unclear. A complete understanding of this synergism was hindered by the commonly believed concept of influenza-induced epithelial damage as the major predisposing factor to secondary bacterial infections. Indeed, some influenza viral virulence factors displayed important roles in this synergism. However, these factors are basically critical for viral pathogenesis, which is a prerequisite for secondary bacterial infections. Thus, this concept would be valid for highly virulent influenza virus strains. However, the findings that even mild seasonal influenza viruses can also enhance the vulnerability to secondary bacterial infections have suggested the existence of more critical mechanisms for this unique synergism drawing the attention to explore possible alterations of the host immunity during influenza infections.

Alterations in host immunity have become an increasingly important aspect of the influenza-mediated predisposition to SBP. Among numerous alterations in host immune responses during influenza infections, defects in pulmonary antibacterial immunity have been observed explaining the strikingly increased vulnerability to bacterial infections. Recently, several mechanisms of the defects in various antibacterial immune effectors have been proposed in influenza-infected lungs [147]. Among those immune effectors, resident AMs play a central role in effective protection against bacterial pathogens in the lungs. 


\section{Impact of Influenza Infections on Resident Alveolar Macrophages In Vivo}

Early studies in the $20^{\text {th }}$ century indicated defective phagocytic functions of AMs during mild and severe influenza infections. [145-148]. Recently, it's been suggested that the AM dysfunction during influenza infections is due to IFN $\gamma$-induced downregulation of the expression of MARCO receptor [101], a scavenger receptor on the surface of AMs, which was implicated in non-opsonized phagocytosis of pneumococci [97]. They proposed this mechanism of defective phagocytosis of AMs only when IFN $\gamma$ secretion is significantly elevated in the lungs, which is temporally related to adaptive immune cell accumulation during the resolution phase of sublethal influenza infection $[101,150]$. However, this mechanism fails to explain the common clinical observations and studies on animal models of bacterial co-infections that showed increased predisposition to secondary bacterial infections during the first week of influenza, even before the appearance of adaptive immune cells and the peak of IFN $\gamma$ production. In addition, another study showed that augmented IFN $\gamma$ production in the lungs can provide protective activity, possibly by increasing neutrophil influx, against lethal pneumococcal pneumonia [205].

Despite the repeated investigation of influenza infection impact on resident AMs, the results were confounding, because most studies were performed using in vitro infection systems, which may not be pathologically relevant to the in vivo influenza infections due to differences in the microenvironment conditions [47, 48, 51]. Even though some investigators have studied the alterations of AM functions in vivo, the misrepresentation of resident AM subset by the total macrophage population harvested in BAL during influenza infections, without strict discrimination approaches, may lead to profoundly erroneous conclusions about the actual state of resident AMs. As a part of the innate immune responses to influenza infections, circulating macrophages and monocytes are recruited to and accumulate in influenza-infected lungs. Therefore, macrophages isolated by BAL process would represent heterogeneous populations of macrophages with different phenotypes. Thus, some investigators implied no change or a significant increase in the numbers of AMs associated with functional phenotype changes during in vivo influenza infections [101, 185].

In contrast, I argue that most of these studies showed erroneous conclusions about the mechanistic influence of influenza infection on resident AMs due to the lack of approaches discriminating resident AMs from the recruited exudate inflammatory macrophages during influenza infection. Thereby, these technical limitations have hindered clear understanding of mechanisms underlying the defects in their protective functions during influenza infections.

To determine the impact of sublethal influenza infection on resident AMs in vivo, I initially characterized different lung-resident macrophage subsets using flow cytometry analyses coupled with a quite novel in vivo labeling approach through intranasal administration of the fluorescent PKH26-PCL dye. This in vivo labeling technique selectively labeled lung-resident macrophages with efficient stability for more than 2 weeks. Comparisons of the fluorescence intensity of the intracellular PKH26-PCL dye 
facilitated successful discrimination between the two major subsets of lung-resident macrophages - AMs and IMs, and could clearly distinguish them from all myeloid subsets recruited during influenza infection.

Surprisingly, I found that sublethal influenza infections using the mouse-adapted PR8 influenza or a human clinical isolate of 2009 pandemic H1N1 influenza virus strains caused significant depletion of resident AMs, but not IMs, with a maximum nadir on day 7 after influenza infection. AM depletion was temporally aligned with the impaired early pneumococcal clearance within the alveolar airspaces from day 3 to day 9 after influenza infection. Later, the AM pool was fully replenished leading to recovery of antibacterial immunity in the lungs. Synergistic interaction between primary influenza and secondary pneumococcal infections was successfully implemented during AM depletion phase, reflected by the progression of lethal secondary pneumococcal pneumonia.

What suggested the causal relationship between AM depletion and increased permissiveness to SBP are the protective outcomes of the immunomodulation approach that we used to expand the pool size of resident AMs during influenza infection. Local pulmonary GM-CSF treatment could significantly expand the pool size of IMs with partial expansion of AMs during influenza infection. These effects could partially restore the protective early bacterial clearance in the lungs and protected co-infected mice against development of SBP.

\section{Insights}

Because of their unique anatomical position at the interface between air and alveolar epithelial cells and continuous exposure to the external environment, resident AMs have a pivotal role for maintaining protection in the lungs against inhaled noxious agents, including environmental particles and bacterial pathogens. Their functions rely on two unique features: high phagocytic capacity and immunosuppressive nature. Therefore, pathological AM depletion during mild influenza infections would have several consequences on the innate and adaptive immune responses in the lungs.

We examined the first obvious consequence of AM depletion during influenza infections which is the expected defect in the first line of pulmonary innate immunity protecting against bacterial pathogens. Indeed, the early bacterial clearance was notably impaired during the AM depletion phase. Pulmonary phagocytic capacity was carefully tested using small inocula of pneumococci rather than big inocula that were used in almost all animal models of secondary bacterial infections following influenza. The use of relatively big inocula of pneumococci for studying protective functions of resident AMs is considered a critical limitation in these animal studies, because these big inocula bypass the phagocytic threshold of resident AMs, and perhaps they are not relevant to clinical settings. In addition, in some studies bacterial clearance was examined at later time points, such as 24 and $48 \mathrm{~h}$ after bacterial inoculation, when neutrophils accumulate as the second line of innate defense. Thereby, they ended up studying neutrophil functions rather than that of resident AMs. 
Consequently, lethal SBP was successfully developed if influenza-infected mice were secondarily infected by small inocula of pneumococci during the AM depletion phase. Taken together, the novel finding of AM depletion during influenza infection implied a novel potential mechanism of the pulmonary viral-bacterial synergism which coincides with the order and timings of SBP in humans [198]. In addition, this mechanism may sound more reasonable to explain the increased vulnerability of influenza-infected hosts to different multiple bacterial pathogens, instead of specific viral-bacterial interactions.

Indeed, two recent studies confirmed our data using completely different, yet elegant, approaches. In the first study, the research group used mice with genetically engineered fluorescent reporter in tissue-resident macrophages, including AMs. They demonstrated dramatic cytoablation of lung-resident macrophages 6 days after sublethal PR8 influenza infection [173]. Furthermore, they also showed that most of the recovery of lung-resident macrophage, after diphtheria toxin-induced depletion, was achieved by stochastic local cellular proliferation in a macrophage colony-stimulating factor (MCSF)- and GM-CSF-dependent manner but independently of IL-4 [173]. These data correlate with our findings about the ontogeny of replenished AMs after influenza infection, which we proposed to be achieved by local proliferation of labeled IMs and their maturation into resident AM phenotype (Figure 2-9).

Additionally, a recent study using mathematical modeling of bacterial coinfection, with the same infectious agents as in our model, expected significant loss of about $90 \%$ of AM functions by day 7 after sublethal PR8 influenza infection [250]. We experimentally demonstrated the same results with depletion of about $90 \%$ of resident AMs on day 7 after sublethal PR8 influenza infection. Furthermore, they hypothesized that this AM dysfunction is one of the major mechanisms underlying the increased influenza-pneumococcal co-infection synergism supporting our experimental data [250].

There may also be other effects of AM depletion during influenza infections, which may not be related to their phagocytic functions, rather stem from their immunosuppressive activities demonstrated in several in vitro and in vivo studies. They display poor antigen presenting abilities and consequently poor $\mathrm{T}$ cell stimulation, as well as inhibitory effects on local DC functions [175-178]. Recently, lung-resident macrophages were shown to be critical mediators of the airway immune tolerance through inducing the generation of Foxp ${ }^{+}$regulatory $\mathrm{T}$ cells in a TGF- $\beta$ - and retinoic acid-dependent manner [251]. Indeed, artificial depletion of pulmonary macrophages using clodronate liposomes abrogated the immunosuppressive state while augmented adaptive immune responses in the lungs [252]. In addition, their artificial depletion was shown to increase secondary IgE responses and infiltration of B cells and T cells into the lungs after allergen exposure [253], as well as increase NK cells and functionality of $\mathrm{CD}^{+}$and $\mathrm{CD} 8^{+} \mathrm{T}$ cells during respiratory pneumovirus infection in mice [254]. Furthermore, a recent study using a murine model of ovalbumin-induced acute asthma demonstrated that clodronate-mediated depletion of AMs increased the airway infiltration of inflammatory immune cells, eosinophils and lymphocytes as well [255]. In contrast, 
the allergic airway inflammation and hyperresponsiveness were attenuated after adoptive transfer of naive AMs $[255,256]$. However, liposome-encapsulated clodronate may also induce depletion of IMs along with AMs, which may complicate the results, as IMs have stronger antigen presenting abilities and stimulatory functions than AMs. In contrast, pathological depletion of AM subset alone may have even more profound impact on the adaptive immune responses and allergic reactions in influenza-infected lungs.

Based on the kinetics of the adaptive immune responses during influenza infections, the peak of their cellular effectors recruitment and functionality in the lungs is quite coincident with the nadir of AM depletion at the end of the first week of influenza infection. This correlation may give novel insights about a probable role of AM depletion in releasing the immunosuppressive brakes in the lungs. Thereby, allowing an efficient functionality of influenza-specific adaptive immune cells in the lungs.

Among other probable outcomes of AM depletion during influenza infections is the influence on the progression of airway hypersensitivity and asthma by possibly lowering the activation threshold for allergen-specific immune cells. Indeed, influenza infections in infants have been recognized as a potent risk factor for asthma development later. Furthermore, asthma exacerbation has been well documented during seasonal and pandemic influenza infections and other respiratory viral infections $[55,257,258]$. In addition, a recent study, in an infant mouse allergy model using house dust mite, demonstrated exacerbation of airway allergic responses when they get infected by influenza viruses [259]. Yet, the mechanisms underlying this synergism have not been completely elucidated.

\section{Future Directions}

The question of how influenza viruses cause depletion of resident AMs in vivo still needs to be fully answered. Indeed, some previous studies showed direct influenza infection of AMs, followed by apoptosis [260, 261]. However, infection was observed in only a small percentage of AMs, and thus cannot totally account for the significant depletion observed. In contrast, some investigators showed no apoptotic events of AMs during influenza infections in pigs [262]. I believe that this significant AM depletion during sublethal influenza infections might have resulted from changes in their key survival signals in the lung microenvironment. These survival signals are probably altered and/or reduced by the damage and death of influenza-infected epithelial cells. To address this possibility, cross-talk between resident AMs and respiratory epithelial cells needs to be explored carefully and tested for major alterations during influenza infections. In addition, it is critical to determine the type of resident AM cell death in vivo during influenza infection, which may elucidate signals causing this depletion process. Generally, there are several ways to determine the type of cell death, including microscopic examination of the morphological changes in the cellular structures to differentiate, for example, between apoptotic and non-apoptotic cell death events. Also, monitoring certain biochemical changes inside the cells may indicate the type of cell death. However, these methods may have some intrinsic technical limitations leading to 
detecting cell death only under limited experimental settings. Also, some techniques may not be able to define the exact cell death type, as the detected features may overlap with various types of cell death $[263,264]$. Therefore, several techniques with unrelated methodologic principles should be performed for better assessment of the type of cell death.

Different respiratory viral infections have been associated with secondary bacterial infections. Although we found significant depletion of the resident AMs during sublethal influenza infections using two different strains of $\mathrm{H} 1 \mathrm{~N} 1$ influenza A viruses as well as another influenza B virus strain, it will be essential to determine the impact of in vivo infections by other influenza A virus subtypes on lung-resident macrophages. In addition, it will be of paramount importance to test if other respiratory viruses, known to increase predisposition to SBP such as respiratory syncytial virus and parainfluenza viruses, can also induce resident AM depletion. If this novel mechanism is observed with different respiratory viruses, it will suggest AM depletion as a general mechanism for the increased respiratory viral-bacterial synergism. Thus, exploring new prophylactic and therapeutic interventions targeting this mechanism would be of great significance to reduce the global burden of SBP during seasonal influenza and any future influenza pandemics. In addition, testing different influenza virus strains for AM depletion ability may infer novel features contributing to viral infection outcomes away from the classical viral virulence determinants.

Another serious clinical aspect associated with influenza infections is the increased risk for airway hyperresponsiveness and asthma exacerbation. Therefore, establishing animal models of influenza infections concomitant with acute or chronic asthma pathogenesis may reveal novel interactions elicited by the loss of lung immunosuppressive state during resident AM depletion.

\section{Treatment Strategies of Secondary Bacterial Pneumonia Following Influenza}

Acute lower respiratory tract infections represent a major public health problem causing significant clinical and socioeconomic burdens worldwide. Compared to other serious infections, they are the leading cause of morbidity and mortality in the United States [120]. Pneumonia is caused by the infection of the alveolar airspace, associated with accumulation of inflammatory cells and secretions in the alveoli causing acute inflammatory lung injury and impaired gas exchange. Treatment of this serious medical problem has been challenging. Despite the wide use of antibiotics since the advent of penicillin to the clinical use in 1940s, the significant mortality rates of influenza and pneumonia patients have persisted with minor changes for more than 50 years $[119,120]$. Many patients progress from uncomplicated respiratory viral infections to severe complicated pneumonia with a high case fatality rate despite adequate antibiotic use, such as patients with SBP following influenza [212]. A recent example of this treatment failure was evident during the $2009 \mathrm{H} 1 \mathrm{~N} 1$ influenza pandemic, in which about one third of the severe or fatal cases were complicated by SBP [35]. Therefore, in addition to new antibiotic classes, we definitely need a non-antibiotic approach for the effective 
management of pneumonia. This will require a better understanding of lung immunopathology and the poor outcomes during antibiotic treatment of pneumonic patients.

One of the enduring questions in this field has been whether adjunctive corticosteroid treatment of pneumonia can improve the outcomes. [227, 239]. The answer to this question has been perplexed by the mixed results shown in several clinical studies of adjunctive corticosteroid use for treating pneumonia in humans [220, 221, 223, 224, 240-242]. Some of these clinical trials showed beneficial effects, while others showed detrimental or no effect after combining corticosteroids with the standard antibiotic treatment of pneumonia.

Using a well-established mouse model of SBP following influenza infection, we tested the hypothesis that adjunctive corticosteroid therapy can modulate antibioticinduced immunopathology during treatment of severe cases of SBP. We also tested the impact of early corticosteroid treatment on primary influenza infection and the outcomes of ampicillin treatment of SBP under these settings. We could monitor the progression of SBP in vivo using bioluminescence imaging, which is a non-invasive accurate surrogate measure of the pneumococcal burden in the lungs. Thus, prior to antibiotic treatment, we classified the pneumonic mice into groups with mild or severe SBP based on the pulmonary bacterial burden.

First, we found an interesting direct correlation between the pneumococcal burdens in the lungs at the onset of ampicillin treatment and the treatment outcomes. Larger bacterial burdens generated more inflammation upon ampicillin-mediated bacterial cell lysis. The release of copious amounts of bacterial PAMPs aggravated the ongoing inflammatory responses in the lungs by activating the inflammatory signaling pathways of different PRRs, including TLR2 detecting pneumococcal cell wall fragments [204]. Thus, the increased lung immunopathology associated with antibiotic treatment of mice with severe SBP eventually caused higher mortality.

Additionally, adjunctive dexamethasone therapy could significantly rescue severe cases of SBP after dampening ampicillin treatment-induced inflammation in the lungs and improving pulmonary vascular permeability. However, it worsened the morbidity of mice with mild SBP reflected by delayed recovery of their body weight.

On the other hand, the early start of dexamethasone therapy during influenza infection resulted in loss of its protective functions. Corticosteroids do not only display potent anti-inflammatory activities, but can also cause systemic immunosuppression $[219,235]$. We examined if early dexamethasone therapy had detrimental impact on the adaptive immunity against primary influenza infection. Indeed, early dexamethasone treatment significantly reduced numbers of $\mathrm{CD} 8^{+}$and $\mathrm{CD} 4^{+} \mathrm{T}$ cells in the lungs on day 7 after influenza infection. This was associated with delayed influenza viral clearance and consequently increasing viral-mediated lung damage, which was implied by the accelerated body weight loss before secondary pneumococcal challenge. 
We also tested the efficacy of DABA, a novel immunomodulator that was shown to attenuate endotoxin-induced acute lung injury [230]. Combined DABA therapy with ampicillin treatment of SBP demonstrated protective effect similar to that of dexamethasone. However, it did not have a negative effect on the morbidity of mice with mild SBP after either adjunctive or early DABA treatment regimen. Nonetheless, its early administration during influenza infection was associated with partial loss of its protective functions.

\section{Insights}

Our findings highlighted several important aspects about the efficacy of adjunctive corticosteroid therapy in CAP, which may give insights explaining part of the discrepancies in the clinical studies of corticosteroid use in CAP. The first aspect is the impact of the severity of pneumonia and the bacterial load size in the lungs at the onset of antibiotic treatment on the adjunctive therapy outcomes. Indeed, the differential treatment outcomes in our murine model of SBP are consistent with several randomized controlled clinical trials that demonstrated positive outcomes of adjunctive corticosteroid therapy only in the severe cases of CAP in humans [220, 221, 241, 242]. However, the criterion that we used for defining the severity in our murine model of SBP relies on the bacterial burden in the lungs, different from the clinical criteria for severe CAP in humans, which depend mostly on the lung functions.

Another critical aspect is the timing of dexamethasone therapy. Our experiments suggested that early dexamethasone treatment during the respiratory viral infections, such as influenza, would be associated with detrimental outcomes due to its suppressive effects on the anti-viral adaptive immunity. This aspect would be of paramount importance in patients with CAP that have simultaneous viral and bacterial etiologies. Therefore, we propose that the best treatment outcomes can be achieved if dexamethasone is administered during the resolution phase of the primary viral infection in conjunction with antibiotic treatment of the complicating bacterial pathogen. Taken together, adjunctive corticosteroid therapy can be used with great efficacy in the severe cases of CAP with careful consideration of the timing of treatment and the onset of any suspected primary viral infection. These findings will be interesting to the trans lational researchers, clinicians, and public health officials for better therapeutic management of lower respiratory tract infections.

\section{Future Directions}

Our findings showed that the murine model of SBP can be used as a good surrogate for preliminary studies on novel immunomodulation approaches during antibiotic treatment of CAP. This model showed results that were clinically relevant to the randomized clinical trials of adjunctive corticosteroid therapy of CAP in humans. Moreover, in vivo bioluminescence imaging proved to be an accurate powerful tool for monitoring the bacterial outgrowth as well as the bacterial clearance in the lungs after 
antibiotic treatment, which was also showed in several previous studies [202, 204, 265, 266]. Therefore, our murine model of SBP and the powerful bioluminescence imaging tool would form a very useful technical approach for objective evaluation of the efficacy of novel prophylactic and therapeutic interventions for treating SBP following influenza.

Indeed, we started exploring novel immunomodulators that can be used for either preventing the increased lung immunopathology elicited during antibiotic treatment of SBP, or promoting the lung tissue repair processes. DABA is a novel immunomodulator that could improve survival of antibiotic-treated mice with severe SBP when given as adjunctive therapy. Furthermore, it did not affect body weight recovery after treatment, which was a potential drawback of adjunctive dexamethasone treatment of mice with mild SBP. DHBA, the active form of DABA, has multiple activities, such as strong iron chelation and free radical scavenging. DHBA has also been shown to inhibit the activity of matrix metalloproteinase-2 (MMP-2), which may help ameliorate the lung tissue damage during pneumonia [267]. MMP-2 is a member of the matrix metalloproteinases (MMPs) that have diverse catalytic functions, mainly facilitating degradation of different components of the extracellular matrix required for tissue remodeling [268]. Interestingly, increased levels of some MMPs were strongly correlated with the clinical severity of CAP and were suggested to contribute to increased lung tissue destruction [269]. Therefore, the probable MMP-inhibitory functions of DABA may mediate its protective functions during treatment of severe SBP. However, further investigation is needed to determine the exact mechanism of action of DABA treatment. In addition, exploring other immunodulators is crucial to help prevent the antibiotic treatment failure in recuing severely pneumonic patients by suppressing the exuberant inflammatory responses in the lungs and/or promoting the lung tissue repair process. 


\section{LIST OF REFERENCES}

1. A revision of the system of nomenclature for influenza viruses: a WHO memorandum. Bull World Health Organ 1980; 58:585-91.

2. Rohm C, Zhou N, Suss J, Mackenzie J, Webster RG. Characterization of a novel influenza hemagglutinin, H15: criteria for determination of influenza A subtypes. Virology 1996; 217:508-16.

3. Fouchier RA, Munster V, Wallensten A, et al. Characterization of a novel influenza A virus hemagglutinin subtype (H16) obtained from black-headed gulls. J Virol 2005; 79:2814-22.

4. Bouvier NM, Palese P. The biology of influenza viruses. Vaccine 2008; 26 Suppl 4:D49-53.

5. Webster RG, Bean WJ, Gorman OT, Chambers TM, Kawaoka Y. Evolution and ecology of influenza A viruses. Microbiol Rev 1992; 56:152-79.

6. Neumann G, Chen H, Gao GF, Shu Y, Kawaoka Y. H5N1 influenza viruses: outbreaks and biological properties. Cell Res 2010; 20:51-61.

7. Olsen B, Munster VJ, Wallensten A, Waldenstrom J, Osterhaus AD, Fouchier RA. Global patterns of influenza a virus in wild birds. Science 2006; 312:384-8.

8. Ducatez MF, Webster RG, Webby RJ. Animal influenza epidemiology. Vaccine 2008; 26 Suppl 4:D67-9.

9. Chen W, Calvo PA, Malide D, et al. A novel influenza A virus mitochondrial protein that induces cell death. Nat Med 2001; 7:1306-12.

10. Pinto LH, Holsinger LJ, Lamb RA. Influenza virus M2 protein has ion channel activity. Cell 1992; 69:517-28.

11. Mackay. IM. Virology Down Under, http://www.uq.edu.au/vdu/ (Last accessed on September 5, 2013).

12. Jagger BW, Wise HM, Kash JC, et al. An overlapping protein-coding region in influenza A virus segment 3 modulates the host response. Science 2012; 337:199204.

13. Rogers GN, Paulson JC, Daniels RS, Skehel JJ, Wilson IA, Wiley DC. Single amino acid substitutions in influenza haemagglutinin change receptor binding specificity. Nature $1983 ; 304: 76-8$.

14. Couceiro JN, Paulson JC, Baum LG. Influenza virus strains selectively recognize sialyloligosaccharides on human respiratory epithelium; the role of the host cell in selection of hemagglutinin receptor specificity. Virus Res 1993; 29:155-65.

15. Matrosovich MN, Matrosovich TY, Gray T, Roberts NA, Klenk HD. Human and avian influenza viruses target different cell types in cultures of human airway epithelium. Proc Natl Acad Sci U S A 2004; 101:4620-4.

16. Suzuki Y. Sialobiology of influenza: molecular mechanism of host range variation of influenza viruses. Biol Pharm Bull 2005; 28:399-408.

17. Stevens J, Blixt O, Tumpey TM, Taubenberger JK, Paulson JC, Wilson IA. Structure and receptor specificity of the hemagglutinin from an H5N1 influenza virus. Science 2006; 312:404-10. 
18. Kuchipudi SV, Nelli R, White GA, Bain M, Chang KC, Dunham S. Differences in influenza virus receptors in chickens and ducks: Implications for interspecies transmission. J Mol Genet Med 2009; 3:143-51.

19. Sieczkarski SB, Whittaker GR. Viral entry. Curr Top Microbiol Immunol 2005; 285:1-23.

20. Martin K, Helenius A. Transport of incoming influenza virus nucleocapsids into the nucleus. J Virol 1991; 65:232-44.

21. Cros JF, Palese P. Trafficking of viral genomic RNA into and out of the nucleus: influenza, Thogoto and Borna disease viruses. Virus Res 2003; 95:3-12.

22. Fujii Y, Goto H, Watanabe T, Yoshida T, Kawaoka Y. Selective incorporation of influenza virus RNA segments into virions. Proc Natl Acad Sci U S A 2003; 100:2002-7.

23. Chou YY, Vafabakhsh R, Doganay S, Gao Q, Ha T, Palese P. One influenza virus particle packages eight unique viral RNAs as shown by FISH analysis. Proc Natl Acad Sci U S A 2012; 109:9101-6.

24. Palese $\mathrm{P}$, Compans $\mathrm{RW}$. Inhibition of influenza virus replication in tissue culture by 2-deoxy-2,3-dehydro-N-trifluoroacetylneuraminic acid (FANA): mechanism of action. J Gen Virol 1976; 33:159-63.

25. Morens DM, North M, Taubenberger JK. Eyewitness accounts of the 1510 influenza pandemic in Europe. Lancet 2010; 376:1894-5.

26. Palese P. Influenza: old and new threats. Nat Med 2004; 10:S82-7.

27. Dawood FS, Iuliano AD, Reed C, et al. Estimated global mortality associated with the first 12 months of 2009 pandemic influenza A H1N1 virus circulation: a modelling study. The Lancet infectious diseases 2012; 12:687-95.

28. Salomon R, Webster RG. The influenza virus enigma. Cell 2009; 136:402-10.

29. Tumpey TM, Garcia-Sastre A, Taubenberger JK, Palese P, Swayne DE, Basler CF. Pathogenicity and immunogenicity of influenza viruses with genes from the 1918 pandemic virus. Proc Natl Acad Sci U S A 2004; 101:3166-71.

30. Kobasa D, Takada A, Shinya K, et al. Enhanced virulence of influenza A viruses with the haemagglutinin of the 1918 pandemic virus. Nature 2004; 431:703-7.

31. Yen HL, Webster RG. Pandemic influenza as a current threat. Curr Top Microbiol Immunol 2009; 333:3-24.

32. Imai M, Watanabe T, Hatta M, et al. Experimental adaptation of an influenza H5 HA confers respiratory droplet transmission to a reassortant $\mathrm{H} 5 \mathrm{HA} / \mathrm{H} 1 \mathrm{~N} 1$ virus in ferrets. Nature 2012; 486:420-8.

33. Herfst S, Schrauwen EJ, Linster M, et al. Airborne transmission of influenza A/H5N1 virus between ferrets. Science 2012; 336:1534-41.

34. Cumulative number of confirmed human cases of avian influenza A (H5N1) reported to WHO: World Health Organization (WHO), 2013.

35. McCullers JA. Preventing and treating secondary bacterial infections with antiviral agents. Antivir Ther 2011; 16:123-35.

36. Chertow DS, Memoli MJ. Bacterial coinfection in influenza: a grand rounds review. JAMA 2013; 309:275-82.

37. Ishii KJ, Koyama S, Nakagawa A, Coban C, Akira S. Host innate immune receptors and beyond: making sense of microbial infections. Cell host \& microbe 2008; 3:352-63. 
38. Takeuchi O, Akira S. Pattern recognition receptors and inflammation. Cell 2010; 140:805-20.

39. Takeuchi $\mathrm{O}$, Akira $\mathrm{S}$. Innate immunity to virus infection. Immunological reviews 2009; 227:75-86.

40. Ichinohe T, Pang IK, Iwasaki A. Influenza virus activates inflammasomes via its intracellular M2 ion channel. Nature immunology 2010; 11:404-10.

41. Allen IC, Scull MA, Moore CB, et al. The NLRP3 inflammasome mediates in vivo innate immunity to influenza A virus through recognition of viral RNA. Immunity 2009; 30:556-65.

42. Thomas PG, Dash P, Aldridge JR, Jr., et al. The intracellular sensor NLRP3 mediates key innate and healing responses to influenza A virus via the regulation of caspase-1. Immunity 2009; 30:566-75.

43. Kreijtz JH, Fouchier RA, Rimmelzwaan GF. Immune responses to influenza virus infection. Virus Res 2011; 162:19-30.

44. Kohlmeier JE, Woodland DL. Immunity to respiratory viruses. Annual review of immunology 2009; 27:61-82.

45. Moltedo B, Lopez CB, Pazos M, Becker MI, Hermesh T, Moran TM. Cutting edge: stealth influenza virus replication precedes the initiation of adaptive immunity. J Immunol 2009; 183:3569-73.

46. Subbarao K, Murphy BR, Fauci AS. Development of effective vaccines against pandemic influenza. Immunity 2006; 24:5-9.

47. Tate MD, Schilter HC, Brooks AG, Reading PC. Responses of mouse airway epithelial cells and alveolar macrophages to virulent and avirulent strains of influenza A virus. Viral Immunol 2011; 24:77-88.

48. van Riel D, Leijten LM, van der Eerden M, et al. Highly pathogenic avian influenza virus $\mathrm{H} 5 \mathrm{~N} 1$ infects alveolar macrophages without virus production or excessive TNF-alpha induction. PLoS pathogens 2011; 7:e1002099.

49. Wijburg OL, DiNatale S, Vadolas J, van Rooijen N, Strugnell RA. Alveolar macrophages regulate the induction of primary cytotoxic $\mathrm{T}$-lymphocyte responses during influenza virus infection. J Virol 1997; 71:9450-7.

50. Tate MD, Pickett DL, van Rooijen N, Brooks AG, Reading PC. Critical role of airway macrophages in modulating disease severity during influenza virus infection of mice. J Virol 2010; 84:7569-80.

51. Wang J, Nikrad MP, Travanty EA, et al. Innate immune response of human alveolar macrophages during influenza A infection. PLoS One 2012; 7:e29879.

52. Neyt K, Lambrecht BN. The role of lung dendritic cell subsets in immunity to respiratory viruses. Immunological reviews 2013; 255:57-67.

53. Aldridge JR, Jr., Moseley CE, Boltz DA, et al. TNF/iNOS-producing dendritic cells are the necessary evil of lethal influenza virus infection. Proc Natl Acad Sci U S A 2009; 106:5306-11.

54. Lin KL, Suzuki Y, Nakano H, Ramsburg E, Gunn MD. CCR2+ monocyte-derived dendritic cells and exudate macrophages produce influenza-induced pulmonary immune pathology and mortality. J Immunol 2008; 180:2562-72.

55. Yoo JK, Kim TS, Hufford MM, Braciale TJ. Viral infection of the lung: Host response and sequelae. The Journal of allergy and clinical immunology 2013. 
56. van de Sandt CE, Kreijtz JH, Rimmelzwaan GF. Evasion of influenza A viruses from innate and adaptive immune responses. Viruses 2012; 4:1438-76.

57. Tumpey TM, Garcia-Sastre A, Taubenberger JK, et al. Pathogenicity of influenza viruses with genes from the 1918 pandemic virus: functional roles of alveolar macrophages and neutrophils in limiting virus replication and mortality in mice. $\mathbf{J}$ Virol 2005; 79:14933-44.

58. Tate MD, Brooks AG, Reading PC. The role of neutrophils in the upper and lower respiratory tract during influenza virus infection of mice. Respiratory research 2008; 9:57.

59. Tate MD, Brooks AG, Reading PC, Mintern JD. Neutrophils sustain effective CD8(+) T-cell responses in the respiratory tract following influenza infection. Immunology and cell biology 2012; 90:197-205.

60. Brandes M, Klauschen F, Kuchen S, Germain RN. A systems analysis identifies a feedforward inflammatory circuit leading to lethal influenza infection. Cell 2013; 154:197-212.

61. Narasaraju T, Yang E, Samy RP, et al. Excessive neutrophils and neutrophil extracellular traps contribute to acute lung injury of influenza pneumonitis. The American journal of pathology 2011; 179:199-210.

62. Lin KL, Sweeney S, Kang BD, Ramsburg E, Gunn MD. CCR2-antagonist prophylaxis reduces pulmonary immune pathology and markedly improves survival during influenza infection. J Immunol 2011; 186:508-15.

63. Subbarao K, Joseph T. Scientific barriers to developing vaccines against avian influenza viruses. Nat Rev Immunol 2007; 7:267-78.

64. Snelgrove RJ, Goulding J, Didierlaurent AM, et al. A critical function for CD200 in lung immune homeostasis and the severity of influenza infection. Nature immunology 2008; 9:1074-83.

65. Carlson CM, Turpin EA, Moser LA, et al. Transforming growth factor-beta: activation by neuraminidase and role in highly pathogenic $\mathrm{H} 5 \mathrm{~N} 1$ influenza pathogenesis. PLoS pathogens 2010; 6:e1001136.

66. Liedmann S, Hrincius ER, Anhlan D, McCullers JA, Ludwig S, Ehrhardt C. New virulence determinants contribute to the enhanced immune response and reduced virulence of an influenza A virus A/PR8/34 variant. J Infect Dis 2013.

67. Fernandez-Sesma A, Marukian S, Ebersole BJ, et al. Influenza virus evades innate and adaptive immunity via the NS1 protein. J Virol 2006; 80:6295-304.

68. Watson DA, Musher DM, Jacobson JW, Verhoef J. A brief history of the pneumococcus in biomedical research: a panoply of scientific discovery. Clin Infect Dis 1993; 17:913-24.

69. Winslow CE, Broadhurst J, Buchanan RE, Krumwiede C, Rogers LA, Smith GH. The Families and Genera of the Bacteria: Final Report of the Committee of the Society of American Bacteriologists on Characterization and Classification of Bacterial Types. Journal of bacteriology 1920; 5:191-229.

70. Griffith F. The Significance of Pneumococcal Types. The Journal of hygiene 1928; 27:113-59. 
71. Avery OT, Macleod CM, McCarty M. Studies on the Chemical Nature of the Substance Inducing Transformation of Pneumococcal Types : Induction of Transformation by a Desoxyribonucleic Acid Fraction Isolated from Pneumococcus Type Iii. The Journal of experimental medicine 1944; 79:137-58.

72. Florey HW. Penicillin; its development for medical uses. Proceedings of the Royal Institution of Great Britain 1946; 33:23-30.

73. Chain E, Florey HW, Adelaide MB, et al. Penicillin as a chemotherapeutic agent. 1940. Clinical orthopaedics and related research 1993:3-7.

74. Laboratory Methods for the Diagnosis of Meningitis caused by Neisseria meningitidis, Streptococcus pneumoniae, and Haemophilus influenzae. Second edition ed: Centers for Disease Control and Prevention and The World Health Organization 2011.

75. Avery OT. A Further Study on the Biologic Classification of Pneumococci. The Journal of experimental medicine 1915; 22:804-19.

76. Kadioglu A, Weiser JN, Paton JC, Andrew PW. The role of Streptococcus pneumoniae virulence factors in host respiratory colonization and disease. Nat Rev Microbiol 2008; 6:288-301.

77. Calix JJ, Porambo RJ, Brady AM, et al. Biochemical, genetic, and serological characterization of two capsule subtypes among Streptococcus pneumoniae Serotype 20 strains: discovery of a new pneumococcal serotype. The Journal of biological chemistry 2012; 287:27885-94.

78. Eldholm V, Johnsborg O, Haugen K, Ohnstad HS, Havarstein LS. Fratricide in Streptococcus pneumoniae: contributions and role of the cell wall hydrolases CbpD, LytA and LytC. Microbiology 2009; 155:2223-34.

79. Martner A, Skovbjerg S, Paton JC, Wold AE. Streptococcus pneumoniae autolysis prevents phagocytosis and production of phagocyte-activating cytokines. Infect Immun 2009; 77:3826-37.

80. McNeela EA, Burke A, Neill DR, et al. Pneumolysin activates the NLRP3 inflammasome and promotes proinflammatory cytokines independently of TLR4. PLoS pathogens 2010; 6:e1001191.

81. Manco S, Hernon F, Yesilkaya H, Paton JC, Andrew PW, Kadioglu A. Pneumococcal neuraminidases A and B both have essential roles during infection of the respiratory tract and sepsis. Infect Immun 2006; 74:4014-20.

82. Weil-Olivier C, van der Linden M, de Schutter I, Dagan R, Mantovani L. Prevention of pneumococcal diseases in the post-seven valent vaccine era: a European perspective. BMC Infect Dis; 12:207.

83. Bogaert D, De Groot R, Hermans PW. Streptococcus pneumoniae colonisation: the key to pneumococcal disease. The Lancet infectious diseases 2004; 4:144-54.

84. Paterson GK, Orihuela CJ. Pneumococci: immunology of the innate host response. Respirology 2010; 15:1057-63.

85. Boyd AR, Shivshankar P, Jiang S, Berton MT, Orihuela CJ. Age-related defects in TLR2 signaling diminish the cytokine response by alveolar macrophages during murine pneumococcal pneumonia. Experimental gerontology 2012; 47:507-18.

86. Zipfel PF, Skerka C. Complement regulators and inhibitory proteins. Nat Rev Immunol 2009; 9:729-40. 
87. Brown JS, Hussell T, Gilliland SM, et al. The classical pathway is the dominant complement pathway required for innate immunity to Streptococcus pneumoniae infection in mice. Proc Natl Acad Sci U S A 2002; 99:16969-74.

88. Mold C, Rodic-Polic B, Du Clos TW. Protection from Streptococcus pneumoniae infection by $\mathrm{C}$-reactive protein and natural antibody requires complement but not Fc gamma receptors. J Immunol 2002; 168:6375-81.

89. Yuste J, Botto M, Paton JC, Holden DW, Brown JS. Additive inhibition of complement deposition by pneumolysin and PspA facilitates Streptococcus pneumoniae septicemia. J Immunol 2005; 175:1813-9.

90. Opitz B, Puschel A, Schmeck B, et al. Nucleotide-binding oligomerization domain proteins are innate immune receptors for internalized Streptococcus pneumoniae. The Journal of biological chemistry 2004; 279:36426-32.

91. Albiger B, Dahlberg S, Sandgren A, et al. Toll-like receptor 9 acts at an early stage in host defence against pneumococcal infection. Cellular microbiology 2007; 9:633-44.

92. Kuroki Y, Takahashi M, Nishitani C. Pulmonary collectins in innate immunity of the lung. Cellular microbiology 2007; 9:1871-9.

93. Knapp S, Schultz MJ, van der Poll T. Pneumonia models and innate immunity to respiratory bacterial pathogens. Shock 2005; 24 Suppl 1:12-8.

94. Franke-Ullmann G, Pfortner C, Walter P, Steinmuller C, Lohmann-Matthes ML, Kobzik L. Characterization of murine lung interstitial macrophages in comparison with alveolar macrophages in vitro. J Immunol 1996; 157:3097-104.

95. Marriott HM, Dockrell DH. The role of the macrophage in lung disease mediated by bacteria. Exp Lung Res 2007; 33:493-505.

96. Dockrell DH, Marriott HM, Prince LR, et al. Alveolar macrophage apoptosis contributes to pneumococcal clearance in a resolving model of pulmonary infection. J Immunol 2003; 171:5380-8.

97. Arredouani M, Yang Z, Ning Y, et al. The scavenger receptor MARCO is required for lung defense against pneumococcal pneumonia and inhaled particles. The Journal of experimental medicine 2004; 200:267-72.

98. Arredouani MS, Yang Z, Imrich A, Ning Y, Qin G, Kobzik L. The macrophage scavenger receptor SR-AI/II and lung defense against pneumococci and particles. Am J Respir Cell Mol Biol 2006; 35:474-8.

99. Sharif O, Matt U, Saluzzo S, et al. The scavenger receptor CD36 downmodulates the early inflammatory response while enhancing bacterial phagocytosis during pneumococcal pneumonia. J Immunol 2013; 190:5640-8.

100. Didierlaurent A, Goulding J, Patel S, et al. Sustained desensitization to bacterial Toll-like receptor ligands after resolution of respiratory influenza infection. The Journal of experimental medicine 2008; 205:323-9.

101. Sun K, Metzger DW. Inhibition of pulmonary antibacterial defense by interferongamma during recovery from influenza infection. Nat Med 2008; 14:558-64.

102. Dockrell DH, Whyte MK, Mitchell TJ. Pneumococcal pneumonia: mechanisms of infection and resolution. Chest 2012; 142:482-91.

103. Yamamoto K, Ahyi AN, Pepper-Cunningham ZA, et al. Roles of Lung Epithelium in Neutrophil Recruitment During Pneumococcal Pneumonia. Am J Respir Cell Mol Biol 2013. 
104. Craig A, Mai J, Cai S, Jeyaseelan S. Neutrophil recruitment to the lungs during bacterial pneumonia. Infect Immun 2009; 77:568-75.

105. Nakasone C, Yamamoto N, Nakamatsu M, et al. Accumulation of gamma/delta T cells in the lungs and their roles in neutrophil-mediated host defense against pneumococcal infection. Microbes and infection / Institut Pasteur 2007; 9:251-8.

106. Hahn I, Klaus A, Janze AK, et al. Cathepsin G and neutrophil elastase play critical and nonredundant roles in lung-protective immunity against Streptococcus pneumoniae in mice. Infect Immun 2011; 79:4893-901.

107. Wartha F, Beiter K, Normark S, Henriques-Normark B. Neutrophil extracellular traps: casting the NET over pathogenesis. Current opinion in microbiology 2007; 10:52-6.

108. van der Poll T, Opal SM. Pathogenesis, treatment, and prevention of pneumococcal pneumonia. Lancet 2009; 374:1543-56.

109. Malley R. Antibody and cell-mediated immunity to Streptococcus pneumoniae: implications for vaccine development. J Mol Med (Berl) 2010; 88:135-42.

110. Casal J, Tarrago D. Immunity to Streptococcus pneumoniae: Factors affecting production and efficacy. Current opinion in infectious diseases 2003; 16:219-24.

111. Snapper CM. Mechanisms underlying in vivo polysaccharide-specific immunoglobulin responses to intact extracellular bacteria. Annals of the New York Academy of Sciences 2012; 1253:92-101.

112. Kadioglu A, Coward W, Colston MJ, Hewitt CR, Andrew PW. CD4-Tlymphocyte interactions with pneumolysin and pneumococci suggest a crucial protective role in the host response to pneumococcal infection. Infect Immun 2004; 72:2689-97.

113. Dworkin MS, Ward JW, Hanson DL, Jones JL, Kaplan JE. Pneumococcal disease among human immunodeficiency virus-infected persons: incidence, risk factors, and impact of vaccination. Clin Infect Dis 2001; 32:794-800.

114. Lu YJ, Gross J, Bogaert D, et al. Interleukin-17A mediates acquired immunity to pneumococcal colonization. PLoS pathogens 2008; 4:e1000159.

115. Malley R, Anderson PW. Serotype-independent pneumococcal experimental vaccines that induce cellular as well as humoral immunity. Proc Natl Acad Sci U S A 2012; 109:3623-7.

116. Tian H, Groner A, Boes M, Pirofski LA. Pneumococcal capsular polysaccharide vaccine-mediated protection against serotype 3 Streptococcus pneumoniae in immunodeficient mice. Infect Immun 2007; 75:1643-50.

117. Weber SE, Tian H, Pirofski LA. CD8+ cells enhance resistance to pulmonary serotype 3 Streptococcus pneumoniae infection in mice. J Immunol 2011; 186:432-42.

118. Jeurissen A, Wuyts M, Kasran A, et al. Essential role for CD40 ligand interactions in $\mathrm{T}$ lymphocyte-mediated modulation of the murine immune response to pneumococcal capsular polysaccharides. J Immunol 2002; 168:277381.

119. Armstrong GL, Conn LA, Pinner RW. Trends in infectious disease mortality in the United States during the 20th century. JAMA 1999; 281:61-6.

120. Mizgerd JP. Lung infection--a public health priority. PLoS medicine 2006; 3:e76. 
121. McCullers JA. Planning for an influenza pandemic: thinking beyond the virus. J Infect Dis 2008; 198:945-7.

122. Johnson NP, Mueller J. Updating the accounts: global mortality of the 1918-1920 "Spanish" influenza pandemic. Bulletin of the history of medicine 2002; 76:10515.

123. Morens DM, Taubenberger JK, Fauci AS. Predominant role of bacterial pneumonia as a cause of death in pandemic influenza: implications for pandemic influenza preparedness. J Infect Dis 2008; 198:962-70.

124. Robertson L, Caley JP, Moore J. Importance of Staphylococcus aureus in pneumonia in the 1957 epidemic of influenza A. Lancet 1958; 2:233-6.

125. Schwarzmann SW, Adler JL, Sullivan RJ, Jr., Marine WM. Bacterial pneumonia during the Hong Kong influenza epidemic of 1968-1969. Archives of internal medicine 1971; 127:1037-41.

126. Rice TW, Rubinson L, Uyeki TM, et al. Critical illness from 2009 pandemic influenza A virus and bacterial coinfection in the United States. Crit Care Med 2012; 40:1487-98.

127. Centers for Disease Control and Prevention. [Summary of notifiable diseases United States, 2009] Published May 13, 2011 for MMWR 2009;58(No. 53):14 http://www.cdc.gov/mmwr/pdf/wk/mm5853.pdf (Last accessed on August 30, 2013).

128. Wang XY, Kilgore PE, Lim KA, et al. Influenza and bacterial pathogen coinfections in the 20th century. Interdiscip Perspect Infect Dis 2011; 2011:146376.

129. McCullers JA. Insights into the interaction between influenza virus and pneumococcus. Clinical microbiology reviews 2006; 19:571-82.

130. Loosli CG. Synergism between respiratory viruses and bacteria. The Yale journal of biology and medicine 1968; 40:522-40.

131. Liderot K, Ahl M, Ozenci V. Secondary bacterial infections in patients with seasonal influenza A and pandemic H1N1. BioMed research international 2013; 2013:376219.

132. Rothberg MB, Haessler SD. Complications of seasonal and pandemic influenza. Crit Care Med 2010; 38:e91-7.

133. Peltola VT, Murti KG, McCullers JA. Influenza virus neuraminidase contributes to secondary bacterial pneumonia. J Infect Dis 2005; 192:249-57.

134. Trappetti C, Kadioglu A, Carter M, et al. Sialic acid: a preventable signal for pneumococcal biofilm formation, colonization, and invasion of the host. J Infect Dis 2009; 199:1497-505.

135. McCullers JA, Iverson AR, McKeon R, Murray PJ. The platelet activating factor receptor is not required for exacerbation of bacterial pneumonia following influenza. Scandinavian journal of infectious diseases 2008; 40:11-7.

136. Bosch AA, Biesbroek G, Trzcinski K, Sanders EA, Bogaert D. Viral and bacterial interactions in the upper respiratory tract. PLoS pathogens 2013; 9:e1003057.

137. Pittet LA, Hall-Stoodley L, Rutkowski MR, Harmsen AG. Influenza virus infection decreases tracheal mucociliary velocity and clearance of Streptococcus pneumoniae. Am J Respir Cell Mol Biol 2010; 42:450-60. 
138. Smith CM, Kulkarni H, Radhakrishnan P, et al. Ciliary dyskinesia is an early feature of respiratory syncytial virus infection. The European respiratory journal : official journal of the European Society for Clinical Respiratory Physiology 2013.

139. McCullers JA. Do specific virus-bacteria pairings drive clinical outcomes of pneumonia? Clin Microbiol Infect 2013; 19:113-8.

140. Tashiro M, Ciborowski P, Klenk HD, Pulverer G, Rott R. Role of Staphylococcus protease in the development of influenza pneumonia. Nature 1987; 325:536-7.

141. Lee RM, White MR, Hartshorn KL. Influenza a viruses upregulate neutrophil tolllike receptor 2 expression and function. Scandinavian journal of immunology 2006; 63:81-9.

142. Miettinen M, Sareneva T, Julkunen I, Matikainen S. IFNs activate toll-like receptor gene expression in viral infections. Genes and immunity 2001; 2:349-55.

143. Dessing MC, van der Sluijs KF, Florquin S, Akira S, van der Poll T. Toll-like receptor 2 does not contribute to host response during postinfluenza pneumococcal pneumonia. Am J Respir Cell Mol Biol 2007; 36:609-14.

144. Jamieson AM, Pasman L, Yu S, et al. Role of tissue protection in lethal respiratory viral-bacterial coinfection. Science 2013; 340:1230-4.

145. Warshauer D, Goldstein E, Akers T, Lippert W, Kim M. Effect of influenza viral infection on the ingestion and killing of bacteria by alveolar macrophages. The American review of respiratory disease 1977; 115:269-77.

146. Nickerson CL, Jakab GJ. Pulmonary antibacterial defenses during mild and severe influenza virus infection. Infect Immun 1990; 58:2809-14.

147. Metzger DW, Sun K. Immune Dysfunction and Bacterial Coinfections following Influenza. J Immunol 2013; 191:2047-52.

148. Jakab GJ. Immune impairment of alveolar macrophage phagocytosis during influenza virus pneumonia. The American review of respiratory disease 1982; 126:778-82.

149. Harris N, Super M, Rits M, Chang G, Ezekowitz RA. Characterization of the murine macrophage mannose receptor: demonstration that the downregulation of receptor expression mediated by interferon-gamma occurs at the level of transcription. Blood 1992; 80:2363-73.

150. Hang do TT, Choi EJ, Song JY, Kim SE, Kwak J, Shin YK. Differential effect of prior influenza infection on alveolar macrophage phagocytosis of Staphylococcus aureus and Escherichia coli: involvement of interferon-gamma production. Microbiology and immunology 2011; 55:751-9.

151. McAuley JL, Chipuk JE, Boyd KL, Van De Velde N, Green DR, McCullers JA. $\mathrm{PB} 1-\mathrm{F} 2$ proteins from $\mathrm{H} 5 \mathrm{~N} 1$ and 20 century pandemic influenza viruses cause immunopathology. PLoS pathogens 2010; 6:e1001014.

152. McAuley JL, Hornung F, Boyd KL, et al. Expression of the 1918 influenza A virus PB1-F2 enhances the pathogenesis of viral and secondary bacterial pneumonia. Cell host \& microbe 2007; 2:240-9.

153. Alymova IV, Green AM, van de Velde N, et al. Immunopathogenic and antibacterial effects of $\mathrm{H} 3 \mathrm{~N} 2$ influenza A virus PB1-F2 map to amino acid residues 62, 75, 79, and 82. J Virol 2011; 85:12324-33. 
154. Weeks-Gorospe JN, Hurtig HR, Iverson AR, et al. Naturally occurring swine influenza A virus PB1-F2 phenotypes that contribute to superinfection with Gram-positive respiratory pathogens. J Virol 2012; 86:9035-43.

155. Coleman JR. The PB1-F2 protein of Influenza A virus: increasing pathogenicity by disrupting alveolar macrophages. Virology journal 2007; 4:9.

156. Small CL, Shaler CR, McCormick S, et al. Influenza infection leads to increased susceptibility to subsequent bacterial superinfection by impairing NK cell responses in the lung. J Immunol 2010; 184:2048-56.

157. Kerr AR, Kirkham LA, Kadioglu A, et al. Identification of a detrimental role for NK cells in pneumococcal pneumonia and sepsis in immunocompromised hosts. Microbes and infection / Institut Pasteur 2005; 7:845-52.

158. Small CL, McCormick S, Gill N, et al. NK cells play a critical protective role in host defense against acute extracellular Staphylococcus aureus bacterial infection in the lung. J Immunol 2008; 180:5558-68.

159. Colamussi ML, White MR, Crouch E, Hartshorn KL. Influenza A virus accelerates neutrophil apoptosis and markedly potentiates apoptotic effects of bacteria. Blood 1999; 93:2395-403.

160. McNamee LA, Harmsen AG. Both influenza-induced neutrophil dysfunction and neutrophil-independent mechanisms contribute to increased susceptibility to a secondary Streptococcus pneumoniae infection. Infect Immun 2006; 74:6707-21.

161. van der Sluijs KF, van Elden LJ, Nijhuis M, et al. IL-10 is an important mediator of the enhanced susceptibility to pneumococcal pneumonia after influenza infection. J Immunol 2004; 172:7603-9.

162. Shahangian A, Chow EK, Tian X, et al. Type I IFNs mediate development of postinfluenza bacterial pneumonia in mice. The Journal of clinical investigation 2009; 119:1910-20.

163. Li W, Moltedo B, Moran TM. Type I interferon induction during influenza virus infection increases susceptibility to secondary Streptococcus pneumoniae infection by negative regulation of gammadelta T cells. J Virol 2012; 86:1230412.

164. Kash JC, Walters KA, Davis AS, et al. Lethal synergism of 2009 pandemic H1N1 influenza virus and Streptococcus pneumoniae coinfection is associated with loss of murine lung repair responses. mBio 2011; 2 .

165. Linares J, Ardanuy C, Pallares R, Fenoll A. Changes in antimicrobial resistance, serotypes and genotypes in Streptococcus pneumoniae over a 30-year period. Clin Microbiol Infect 2010; 16:402-10.

166. Musher DM, Dowell ME, Shortridge VD, et al. Emergence of macrolide resistance during treatment of pneumococcal pneumonia. The New England journal of medicine 2002; 346:630-1.

167. Hayden FG, de Jong MD. Emerging influenza antiviral resistance threats. J Infect Dis 2011; $203: 6-10$.

168. Ling LM, Chow AL, Lye DC, et al. Effects of early oseltamivir therapy on viral shedding in 2009 pandemic influenza A (H1N1) virus infection. Clin Infect Dis 2010; 50:963-9. 
169. Holt PG, Strickland DH, Wikstrom ME, Jahnsen FL. Regulation of immunological homeostasis in the respiratory tract. Nat Rev Immunol 2008; 8:142-52.

170. Myrvik Q, Leake ES, Fariss B. Studies on pulmonary alveolar macrophages from the normal rabbit: a technique to procure them in a high state of purity. J Immunol 1961; 86:128-32.

171. Lohmann-Matthes ML, Steinmuller C, Franke-Ullmann G. Pulmonary macrophages. The European respiratory journal : official journal of the European Society for Clinical Respiratory Physiology 1994; 7:1678-89.

172. Landsman L, Jung S. Lung macrophages serve as obligatory intermediate between blood monocytes and alveolar macrophages. J Immunol 2007; 179:3488-94.

173. Hashimoto D, Chow A, Noizat C, et al. Tissue-resident macrophages selfmaintain locally throughout adult life with minimal contribution from circulating monocytes. Immunity 2013; 38:792-804.

174. Jenkins SJ, Ruckerl D, Cook PC, et al. Local macrophage proliferation, rather than recruitment from the blood, is a signature of TH2 inflammation. Science 2011; 332:1284-8.

175. Holt PG, Oliver J, Bilyk N, et al. Downregulation of the antigen presenting cell function(s) of pulmonary dendritic cells in vivo by resident alveolar macrophages. The Journal of experimental medicine 1993; 177:397-407.

176. Blumenthal RL, Campbell DE, Hwang P, DeKruyff RH, Frankel LR, Umetsu DT. Human alveolar macrophages induce functional inactivation in antigen-specific CD4 T cells. The Journal of allergy and clinical immunology 2001; 107:258-64.

177. Jakubzick C, Tacke F, Llodra J, van Rooijen N, Randolph GJ. Modulation of dendritic cell trafficking to and from the airways. J Immunol 2006; 176:3578-84.

178. Upham JW, Strickland DH, Robinson BW, Holt PG. Selective inhibition of T cell proliferation but not expression of effector function by human alveolar macrophages. Thorax 1997; 52:786-95.

179. MacLean JA, Xia W, Pinto CE, Zhao L, Liu HW, Kradin RL. Sequestration of inhaled particulate antigens by lung phagocytes. A mechanism for the effective inhibition of pulmonary cell-mediated immunity. The American journal of pathology 1996; 148:657-66.

180. Minino AM, Heron MP, Murphy SL, Kochanek KD. Deaths: final data for 2004. Natl Vital Stat Rep 2007; 55:1-119.

181. Liu L, Johnson HL, Cousens S, et al. Global, regional, and national causes of child mortality: an updated systematic analysis for 2010 with time trends since 2000. Lancet 2012; 379:2151-61.

182. Song JH, Thamlikitkul V, Hsueh PR. Clinical and economic burden of community-acquired pneumonia amongst adults in the Asia-Pacific region. International journal of antimicrobial agents 2011; 38:108-17.

183. McCullers JA, English BK. Improving therapeutic strategies for secondary bacterial pneumonia following influenza. Future microbiology 2008; 3:397-404.

184. Lipscomb MF, Hutt J, Lovchik J, Wu T, Lyons CR. The pathogenesis of acute pulmonary viral and bacterial infections: investigations in animal models. Annual review of pathology 2010; 5:223-52. 
185. Herold S, Steinmueller M, von Wulffen W, et al. Lung epithelial apoptosis in influenza virus pneumonia: the role of macrophage-expressed TNF-related apoptosis-inducing ligand. The Journal of experimental medicine 2008; 205:306577.

186. Bradley LM, Douglass MF, Chatterjee D, Akira S, Baaten BJ. Matrix metalloprotease 9 mediates neutrophil migration into the airways in response to influenza virus-induced toll-like receptor signaling. PLoS pathogens 2012; 8:e1002641.

187. Bedoret $\mathrm{D}$, Wallemacq $\mathrm{H}$, Marichal $\mathrm{T}$, et al. Lung interstitial macrophages alter dendritic cell functions to prevent airway allergy in mice. The Journal of clinical investigation 2009; 119:3723-38.

188. Guth AM, Janssen WJ, Bosio CM, Crouch EC, Henson PM, Dow SW. Lung environment determines unique phenotype of alveolar macrophages. Am J Physiol Lung Cell Mol Physiol 2009; 296:L936-46.

189. Janssen WJ, Barthel L, Muldrow A, et al. Fas determines differential fates of resident and recruited macrophages during resolution of acute lung injury. Am J Respir Crit Care Med 2011; 184:547-60.

190. Gonzalez-Juarrero M, Shim TS, Kipnis A, Junqueira-Kipnis AP, Orme IM. Dynamics of macrophage cell populations during murine pulmonary tuberculosis. J Immunol 2003; 171:3128-35.

191. Auffray C, Sieweke MH, Geissmann F. Blood monocytes: development, heterogeneity, and relationship with dendritic cells. Annual review of immunology 2009; 27:669-92.

192. Gordon S, Taylor PR. Monocyte and macrophage heterogeneity. Nat Rev Immunol 2005; 5:953-64.

193. Murray PJ, Wynn TA. Protective and pathogenic functions of macrophage subsets. Nat Rev Immunol 2011; 11:723-37.

194. Chamoto K, Gibney BC, Ackermann M, et al. Alveolar macrophage dynamics in murine lung regeneration. Journal of cellular physiology 2012; 227:3208-15.

195. Zaslona Z, Wilhelm J, Cakarova L, et al. Transcriptome profiling of primary murine monocytes, lung macrophages and lung dendritic cells reveals a distinct expression of genes involved in cell trafficking. Respiratory research 2009; 10:2.

196. Lehnert BE. Pulmonary and thoracic macrophage subpopulations and clearance of particles from the lung. Environ Health Perspect 1992; 97:17-46.

197. McCullers JA, Rehg JE. Lethal synergism between influenza virus and Streptococcus pneumoniae: characterization of a mouse model and the role of platelet-activating factor receptor. J Infect Dis 2002; 186:341-50.

198. Brundage JF, Shanks GD. Deaths from bacterial pneumonia during 1918-19 influenza pandemic. Emerging infectious diseases 2008; 14:1193-9.

199. Duan M, Li WC, Vlahos R, Maxwell MJ, Anderson GP, Hibbs ML. Distinct macrophage subpopulations characterize acute infection and chronic inflammatory lung disease. J Immunol 2012; 189:946-55.

200. Hashimoto Y, Moki T, Takizawa T, Shiratsuchi A, Nakanishi Y. Evidence for phagocytosis of influenza virus-infected, apoptotic cells by neutrophils and macrophages in mice. J Immunol 2007; 178:2448-57. 
201. Habibzay M, Weiss G, Hussell T. Bacterial superinfection following lung inflammatory disorders. Future microbiology 2013; 8:247-56.

202. Karlstrom A, Boyd KL, English BK, McCullers JA. Treatment with protein synthesis inhibitors improves outcomes of secondary bacterial pneumonia after influenza. J Infect Dis 2009; 199:311-9.

203. Francis KP, Yu J, Bellinger-Kawahara C, et al. Visualizing pneumococcal infections in the lungs of live mice using bioluminescent Streptococcus pneumoniae transformed with a novel gram-positive lux transposon. Infect Immun 2001; 69:3350-8.

204. Karlstrom A, Heston SM, Boyd KL, Tuomanen EI, McCullers JA. Toll-like receptor 2 mediates fatal immunopathology in mice during treatment of secondary pneumococcal pneumonia following influenza. J Infect Dis 2011; 204:1358-66.

205. Sun K, Salmon SL, Lotz SA, Metzger DW. Interleukin-12 promotes gamma interferon-dependent neutrophil recruitment in the lung and improves protection against respiratory Streptococcus pneumoniae infection. Infect Immun 2007; 75:1196-202.

206. Shibata Y, Berclaz PY, Chroneos ZC, Yoshida M, Whitsett JA, Trapnell BC. GM-CSF regulates alveolar macrophage differentiation and innate immunity in the lung through PU.1. Immunity 2001; 15:557-67.

207. Huang FF, Barnes PF, Feng Y, et al. GM-CSF in the lung protects against lethal influenza infection. Am J Respir Crit Care Med 2011; 184:259-68.

208. Yoshida M, Ikegami M, Reed JA, Chroneos ZC, Whitsett JA. GM-CSF regulates protein and lipid catabolism by alveolar macrophages. Am J Physiol Lung Cell Mol Physiol 2001; 280:L379-86.

209. Sever-Chroneos Z, Murthy A, Davis J, et al. GM-CSF modulates pulmonary resistance to influenza A infection. Antiviral research 2011; 92:319-28.

210. Steinwede K, Tempelhof O, Bolte K, et al. Local delivery of GM-CSF protects mice from lethal pneumococcal pneumonia. J Immunol 2011; 187:5346-56.

211. Hamilton JA. Colony-stimulating factors in inflammation and autoimmunity. Nat Rev Immunol 2008; 8:533-44.

212. Palacios G, Hornig M, Cisterna D, et al. Streptococcus pneumoniae coinfection is correlated with the severity of H1N1 pandemic influenza. PLoS One 2009; 4:e8540.

213. Dagan R, Bhutta ZA, de Quadros CA, et al. The remaining challenge of pneumonia: the leading killer of children. Pediatr Infect Dis J 2011; 30:1-2.

214. Bautista E, Chotpitayasunondh T, Gao Z, et al. Clinical aspects of pandemic 2009 influenza A (H1N1) virus infection. The New England journal of medicine 2010; 362:1708-19.

215. Estenssoro E, Rios FG, Apezteguia C, et al. Pandemic 2009 influenza A in Argentina: a study of 337 patients on mechanical ventilation. Am J Respir Crit Care Med 2010; 182:41-8.

216. Bradley JS, Byington CL, Shah SS, et al. The management of communityacquired pneumonia in infants and children older than 3 months of age: clinical practice guidelines by the Pediatric Infectious Diseases Society and the Infectious Diseases Society of America. Clin Infect Dis 2011; 53:e25-76. 
217. Mandell LA, Wunderink RG, Anzueto A, et al. Infectious Diseases Society of America/American Thoracic Society consensus guidelines on the management of community-acquired pneumonia in adults. Clin Infect Dis 2007; 44 Suppl 2:S2772.

218. Barnes PJ. How corticosteroids control inflammation: Quintiles Prize Lecture 2005. Br J Pharmacol 2006; 148:245-54.

219. Rhen T, Cidlowski JA. Antiinflammatory action of glucocorticoids--new mechanisms for old drugs. The New England journal of medicine 2005; 353:1711-23.

220. Meijvis SC, Hardeman H, Remmelts HH, et al. Dexamethasone and length of hospital stay in patients with community-acquired pneumonia: a randomised, double-blind, placebo-controlled trial. Lancet 2011; 377:2023-30.

221. Confalonieri M, Urbino R, Potena A, et al. Hydrocortisone infusion for severe community-acquired pneumonia: a preliminary randomized study. Am J Respir Crit Care Med 2005; 171:242-8.

222. Annane D, Sebille V, Charpentier C, et al. Effect of treatment with low doses of hydrocortisone and fludrocortisone on mortality in patients with septic shock. JAMA 2002; 288:862-71.

223. Snijders D, Daniels JM, de Graaff CS, van der Werf TS, Boersma WG. Efficacy of corticosteroids in community-acquired pneumonia: a randomized doubleblinded clinical trial. Am J Respir Crit Care Med 2010; 181:975-82.

224. Marik P, Kraus P, Sribante J, Havlik I, Lipman J, Johnson DW. Hydrocortisone and tumor necrosis factor in severe community-acquired pneumonia. A randomized controlled study. Chest 1993; 104:389-92.

225. World Health Organization (2009) Clinical Management of Human Infection with Pandemic (H1N1) 2009: Revised Guidance. Geneva: World Health Organization. http://www.who.int/csr/resources/publications/swineflu/clinical management h1 n1.pdf (Last accessed on July 23, 2013).

226. Matthay MA, Liu KD. Con: corticosteroids are not indicated for treatment of acute lung injury from H1N1 viral pneumonia. Am J Respir Crit Care Med 2011; 183:1127-8.

227. Confalonieri M, Kodric M, Santagiuliana M, et al. To use or not to use corticosteroids for pneumonia? A clinician's perspective. Monaldi Arch Chest Dis 2012; 77:94-101.

228. Annane D. Pro: the illegitimate crusade against corticosteroids for severe H1N1 pneumonia. Am J Respir Crit Care Med 2011; 183:1125-6.

229. Ghoneim HE, Thomas PG, McCullers JA. Depletion of Alveolar Macrophages during Influenza Infection Facilitates Bacterial Superinfections. J Immunol 2013; 191:1250-9.

230. Eiznhamer DA, Flavin MT, Jesmok GJ, et al. Effective attenuation of endotoxininduced acute lung injury by 2,3-diacetyloxybenzoic acid in two independent animal models. Pulmonary pharmacology \& therapeutics 2004; 17:105-10.

231. Graziano JH, Grady RW, Cerami A. The identification of 2, 3-dihydroxybenzoic acid as a potentially useful iron-chelating drug. The Journal of pharmacology and experimental therapeutics $1974 ; 190: 570-5$. 
232. Lopez-Goni I, Moriyon I, Neilands JB. Identification of 2,3-dihydroxybenzoic acid as a Brucella abortus siderophore. Infect Immun 1992; 60:4496-503.

233. Naghibi B, Ghafghazi T, Hajhashemi V, Talebi A, Taheri D. The effect of 2,3dihydroxybenzoic acid and tempol in prevention of vancomycin-induced nephrotoxicity in rats. Toxicology 2007; 232:192-9.

234. McCullers JA. Effect of antiviral treatment on the outcome of secondary bacterial pneumonia after influenza. J Infect Dis 2004; 190:519-26.

235. Schacke H, Docke WD, Asadullah K. Mechanisms involved in the side effects of glucocorticoids. Pharmacology \& therapeutics 2002; 96:23-43.

236. Austrian R, Gold J. Pneumococcal Bacteremia with Especial Reference to Bacteremic Pneumococcal Pneumonia. Ann Intern Med 1964; 60:759-76.

237. Feikin DR, Schuchat A, Kolczak M, et al. Mortality from invasive pneumococcal pneumonia in the era of antibiotic resistance, 1995-1997. Am J Public Health 2000; 90:223-9.

238. Chiou CC, Yu VL. Severe pneumococcal pneumonia: new strategies for management. Current opinion in critical care 2006; 12:470-6.

239. Meijvis SC, van de Garde EM, Rijkers GT, Bos WJ. Treatment with antiinflammatory drugs in community-acquired pneumonia. J Intern Med 2012; 272:25-35.

240. Siempos, II, Vardakas KZ, Kopterides P, Falagas ME. Adjunctive therapies for community-acquired pneumonia: a systematic review. The Journal of antimicrobial chemotherapy 2008; 62:661-8.

241. Fernandez-Serrano S, Dorca J, Garcia-Vidal C, et al. Effect of corticosteroids on the clinical course of community-acquired pneumonia: a randomized controlled trial. Crit Care 2011; 15:R96.

242. Mikami K, Suzuki M, Kitagawa H, et al. Efficacy of corticosteroids in the treatment of community-acquired pneumonia requiring hospitalization. Lung 2007; 185:249-55.

243. Su X, Wang L, Song Y, Bai C. Inhibition of inflammatory responses by ambroxol, a mucolytic agent, in a murine model of acute lung injury induced by lipopolysaccharide. Intensive care medicine 2004; 30:133-40.

244. Kim J, McKinley L, Siddiqui J, Bolgos GL, Remick DG. Prevention and reversal of pulmonary inflammation and airway hyperresponsiveness by dexamethasone treatment in a murine model of asthma induced by house dust. Am J Physiol Lung Cell Mol Physiol 2004; 287:L503-9.

245. Grohmann U, Volpi C, Fallarino F, et al. Reverse signaling through GITR ligand enables dexamethasone to activate IDO in allergy. Nat Med 2007; 13:579-86.

246. Xu T, Qiao J, Zhao L, et al. Effect of dexamethasone on acute respiratory distress syndrome induced by the $\mathrm{H} 5 \mathrm{~N} 1$ virus in mice. The European respiratory journal : official journal of the European Society for Clinical Respiratory Physiology 2009; 33:852-60.

247. Reagan-Shaw S, Nihal M, Ahmad N. Dose translation from animal to human studies revisited. FASEB journal : official publication of the Federation of American Societies for Experimental Biology 2008; 22:659-61. 
248. Salluh JI, Povoa P, Soares M, Castro-Faria-Neto HC, Bozza FA, Bozza PT. The role of corticosteroids in severe community-acquired pneumonia: a systematic review. Crit Care 2008; 12:R76.

249. Drug Facts \& Comparisons: Pocket Version, 2009 Edition. Chapter 3, Endocrine and Metabolic Agents, page: 171.

250. Smith AM, Adler FR, Ribeiro RM, et al. Kinetics of coinfection with influenza A virus and Streptococcus pneumoniae. PLoS pathogens 2013; 9:e1003238.

251. Soroosh P, Doherty TA, Duan W, et al. Lung-resident tissue macrophages generate Foxp3+ regulatory T cells and promote airway tolerance. The Journal of experimental medicine 2013; 210:775-88

252. Thepen T, McMenamin C, Oliver J, Kraal G, Holt PG. Regulation of immune response to inhaled antigen by alveolar macrophages: differential effects of in vivo alveolar macrophage elimination on the induction of tolerance vs. immunity. Eur J Immunol 1991; 21:2845-50.

253. Thepen T, McMenamin C, Girn B, Kraal G, Holt PG. Regulation of IgE production in pre-sensitized animals: in vivo elimination of alveolar macrophages preferentially increases IgE responses to inhaled allergen. Clinical and experimental allergy : journal of the British Society for Allergy and Clinical Immunology 1992; 22:1107-14.

254. Rigaux P, Killoran KE, Qiu Z, Rosenberg HF. Depletion of alveolar macrophages prolongs survival in response to acute pneumovirus infection. Virology 2012; 422:338-45.

255. Bang BR, Chun E, Shim EJ, et al. Alveolar macrophages modulate allergic inflammation in a murine model of asthma. Experimental \& molecular medicine 2011; 43:275-80.

256. Careau E, Turmel V, Lauzon-Joset JF, Bissonnette EY. Alveolar macrophages reduce airway hyperresponsiveness and modulate cytokine levels. Exp Lung Res 2010; 36:255-61.

257. Holt PG, Sly PD. Viral infections and atopy in asthma pathogenesis: new rationales for asthma prevention and treatment. Nat Med 2012; 18:726-35.

258. Hasegawa S, Hirano R, Hashimoto K, Haneda Y, Shirabe K, Ichiyama T. Characteristics of atopic children with pandemic H1N1 influenza viral infection: pandemic H1N1 influenza reveals 'occult' asthma of childhood. Pediatric allergy and immunology : official publication of the European Society of Pediatric Allergy and Immunology 2011; 22:e119-23.

259. Al-Garawi A, Fattouh R, Botelho F, et al. Influenza A facilitates sensitization to house dust mite in infant mice leading to an asthma phenotype in adulthood. Mucosal immunology 2011; 4:682-94.

260. Xie D, Bai H, Liu L, et al. Apoptosis of lymphocytes and monocytes infected with influenza virus might be the mechanism of combating virus and causing secondary infection by influenza. Int Immunol 2009; 21:1251-62.

261. Kosai K, Seki M, Tanaka A, et al. Increase of apoptosis in a murine model for severe pneumococcal pneumonia during influenza A virus infection. Japanese journal of infectious diseases 2011; 64:451-7. 
262. Seo SH, Webby R, Webster RG. No apoptotic deaths and different levels of inductions of inflammatory cytokines in alveolar macrophages infected with influenza viruses. Virology 2004; 329:270-9.

263. Kepp O, Galluzzi L, Lipinski M, Yuan J, Kroemer G. Cell death assays for drug discovery. Nature reviews Drug discovery 2011; 10:221-37.

264. Galluzzi L, Aaronson SA, Abrams J, et al. Guidelines for the use and interpretation of assays for monitoring cell death in higher eukaryotes. Cell death and differentiation 2009; 16:1093-107.

265. Johnson AW, Sidman JD, Lin J. Bioluminescent imaging of pneumococcal otitis media in chinchillas. The Annals of otology, rhinology, and laryngology 2013; 122:344-52.

266. McCullers JA, Bartmess KC. Role of neuraminidase in lethal synergism between influenza virus and Streptococcus pneumoniae. J Infect Dis 2003; 187:1000-9.

267. Gendron R, Grenier D, Sorsa T, Uitto VJ, Mayrand D. Effect of microbial siderophores on matrix metalloproteinase-2 activity. Journal of periodontal research 1999; 34:50-3.

268. Chakrabarti S, Patel KD. Matrix metalloproteinase-2 (MMP-2) and MMP-9 in pulmonary pathology. Exp Lung Res 2005; 31:599-621.

269. Hartog CM, Wermelt JA, Sommerfeld CO, Eichler W, Dalhoff K, Braun J. Pulmonary matrix metalloproteinase excess in hospital-acquired pneumonia. Am J Respir Crit Care Med 2003; 167:593-8. 


\section{VITA}

Hazem Elsayed Ghoneim was born in Giza, Egypt in1984. He graduated from Elsaideya High School in Giza, Egypt in May 2000. He obtained his bachelor degree in Pharmaceutical Sciences from the Faculty of Pharmacy, Cairo University in May 2005 with distinction and highest honors. After graduation, he was appointed as a teaching and research assistant at the Department of Microbiology and Immunology, Faculty of Pharmacy, Cairo University. In August 2009, he started his PhD scholarship in the Integrated Biomedical Sciences Program at the University of Tennessee Health Science Center in Memphis, Tennessee. He performed his dissertation studies under the supervision of Dr. Jonathan A. McCullers at his laboratory in St. Jude Children's Research Hospital in Memphis, Tennessee. He received his PhD in Microbiology, Immunology, and Biochemistry in December 2013. 